Check for updates

Cite this: Mater. Adv., 2022, 3, 1359

Received 7th September 2021, Accepted 19th November 2021

DOI: 10.1039/d1ma00814e

rsc.li/materials-advances

\title{
Electrocatalysis enabled transformation of earth-abundant water, nitrogen and carbon dioxide for a sustainable future
}

\author{
Kaili Liu, $\dagger^{\mathrm{ab}}$ Pengfei Cao, $\dagger^{\mathrm{C}}$ Wei Chen, $\dagger^{\mathrm{a}}$ Collins I. Ezeh, ${ }^{\mathrm{a}} \mathrm{Zijian}$ Chen, ${ }^{\mathrm{D}}{ }^{\mathrm{a}}$ \\ Yonglan Luo, (D) d Qian Liu, ${ }^{e}$ Haitao Zhao, ${ }^{*}$ Zhenhua Rui, ${ }^{f}$ Shuyan Gao, (D) 9 \\ Zongyou Yin, (D) *b Xuping Sun (D) ${ }^{d}$ and Xuefeng Yu (D) ${ }^{a}$
}

\begin{abstract}
The integration of electrochemistry with catalyst systems underscores a major sustainable scheme for the production of fossil-free fuels and valuable chemicals. This undertaking necessitates the need for rational design of electrocatalysts with high catalytic activity, selectivity, and stability for electrochemical conversion. Significant progress has been made in this regard considering the importance of the products in these reaction systems. Hence, this review presents an update of both experimental and theoretical investigations that can offer insights into the design of high-performance electrocatalysts to facilitate the electrochemical conversion of $\mathrm{H}_{2} \mathrm{O}, \mathrm{N}_{2}$ and $\mathrm{CO}_{2}$ into value added products. We analyse the current status of available electrocatalysts based on a standard set of figures of merit, namely yield rate, faradaic efficiency, overpotential, current density and stability. Then, we constructively compare the different electrocatalysts based on their reaction mechanisms and operation performances by evaluating the catalyst construction, electrolyte utilization and device practicality. Finally, we provide challenges and prospects from the aspects of both theoretical and experimental insights as a general guide to offer potential future directions.
\end{abstract}

\section{Introduction}

Fossil fuels are the primary energy source driving over $86 \%$ of the global anthropogenic effect, not to mention their limited abundance that could only sustain for the next 50 years approximately. ${ }^{1}$ Their continuous consumption will lead to an increase in the level of atmospheric $\mathrm{CO}_{2}$ with a deleterious impact on the climate. To effectively mitigate this impact for a sustainable future, it is critical to replace fossil fuels with

\footnotetext{
${ }^{a}$ Materials Interfaces Center, Shenzhen Institutes of Advanced Technology, Chinese Academy of Sciences, Shenzhen 518055, Guangdong, China. E-mail: ht.zhao@siat.ac.cn

${ }^{b}$ Research School of Chemistry, Australian National University, Canberra, ATC 2601, Australia.E-mail: zongyou.yin@anu.edu.au

${ }^{c}$ School of Chemical Engineering and Technology, Xi'an Jiaotong University, Xi'an 710049, Shanxi, China

${ }^{d}$ Institute of Fundamental and Frontier Sciences, University of Electronic Science and Technology of China, Chengdu 610054, Sichuan, China. E-mail:xpsun@uestc.edu.cn

${ }^{e}$ Institute for Advanced Study, Chengdu University, Chengdu 610106, Sichuan, China

${ }^{f}$ Department of Mechanical Engineering, Massachusetts Institute of Technology, Cambridge, 02139, USA

${ }^{g}$ School of Materials Science and Engineering, Henan Normal University, Xinxiang 453007, Henan, China

$\dagger$ These authors contributed equally to this work.
}

alternative energy sources. ${ }^{2}$ Earth's atmosphere provides a universal feedstock of water, carbon dioxide, and nitrogen which can be converted into hydrogen, hydrocarbons and ammonia, respectively. This would significantly contribute to solve societal problems sustainably in terms of clean energy (hydrogen fuel), chemical sources (hydrocarbon, ammonia) and a clean environment $\left(\mathrm{CO}_{2}\right.$ capturing).

The development of electrochemical technology that can transform earth-abundant molecules into valuable products offers us the chance to directly address the most pressing environmental challenges and energy crisis by decreasing the utilization of fossil fuels while increasing the production of sustainable fuels and chemicals. Unfortunately, the efficiency and/or stability of such electrochemical processes are still not that satisfactory due to the sluggish kinetics of key reactions and/or the catalyst's susceptibility to the working environment. The integration of the electrochemical processes and rationally designed catalysts is essential for the transformation of these abundant molecules with efficient and versatile platforms to store and utilize green energy and/or produce valuable chemicals for other uses. ${ }^{3,4}$

An emerging sustainable scheme considers the coupling of renewable-energy plants and electrocatalytic reactors, where electrocatalysis can enable the high-performance chemical transformation with improved kinetics, efficiency, and selectivity. 
Electrocatalysis is an interdisciplinary area that encompasses the principles of physics, chemistry, and materials science to allow a comprehensive understanding of the reaction kinetics and stability. ${ }^{5}$ Hence, insight into electrocatalysis is an indispensable issue to ensure the scalable transformation of earth-abundant molecules for a sustainable future. ${ }^{6,7}$

The pioneering works to transform these molecules have long been recorded in history. Electrochemical water splitting was demonstrated for the production of hydrogen in 1800 by Nicholson and Carlisle. ${ }^{8}$ Following the first substantiated report on $\mathrm{CO}_{2}$ reduction reaction (CRR) via electrochemistry by Jordan and Smith in $1960,{ }^{9,10}$ Hori and co-workers revealed the possible means of producing carbonaceous chemicals and fuels using this process in $1985 .{ }^{11-13}$ In 1990, electrocatalytic nitrogen reduction reaction to ammonia was reported by Furuya and Yoshiba. ${ }^{14}$ Because of these historical initiatives, more scientific studies adopting these chemical transformations and energy conversion processes have been conducted. Despite the recent development, these electrochemical processes are confronted with shortcomings, limiting their practical implementation. Therefore, it is of paramount importance to carry out a systematic review on the development of electrocatalysts for a sustainable future.

Nowadays, high-quality reviews on the general concept of electrocatalysis ${ }^{3}$ and electrocatalysts for energy-related reactions ${ }^{15}$ and perspectives on electrocatalytic conversion ${ }^{6}$ have been reported addressing the fundamentals and technical issues. However, there is still a paucity of information regarding the comprehensive and critical outlook on advanced electrocatalysts for the transformation of these three molecules (water, nitrogen and carbon dioxide) with a systematic and constructive survey. Moreover, the field is developing very rapidly and hence new progress is being made in understanding the role of electrocatalysts in the key reactions such as water splitting, NRR and CRR. Therefore, this field deserves a timely review encompassing the dynamic advancement of electrocatalysis in transforming earth-abundant molecules into valuable products for a sustainable future.

Hence, this study is a critical review of both experimental and theoretical investigations offering insights into catalysts for electrochemical conversion of essential molecules - water, nitrogen and carbon dioxide - and guiding the design of high-performance catalyst systems necessary to facilitate these conversions. Herein, we analyze the current status of available catalytic materials (metal and metal-free) based on a standard set of figures of merit, namely faradaic efficiency, energy efficiency, overpotential, current density, and stability. This approach enables a fair and insightful comparison among the different electrocatalysts, identifies the limiting phenomena, and forecasts the feasibility of practical applications in a much shorter timeframe.

\section{Hydrogen production via water splitting}

Hydrogen energy is a carbon-neutral resource for replacing fossil fuels in the future and the 'hydrogen economy' calls for efficient processes for the production of hydrogen from renewables. ${ }^{16,17}$ So far, there are three recognized approaches to produce hydrogen: water splitting, steam reformation of methane and coal gasification. ${ }^{1}$ Among them, water splitting is a spotless pathway and indeed represents the most sustainable strategy if backed up by renewable resources. Yet, this route accounts only for approximately $1 \%$ of the overall hydrogen production in the world. ${ }^{18-20}$ To function effectively, water splitting has been pursuing the development of catalysts that can stabilize and accelerate the reaction along a particular route. Catalysts with good charge transfer, high activity, high selectivity and extensive stability are desired. ${ }^{21-23}$ Electrocatalysts have been widely employed to enhance the conversion efficiency of water to $\mathrm{H}_{2}$ and $\mathrm{O}_{2} \cdot{ }^{24,25}$ Herein, we discuss the chemistry underlying the electrosynthesis of $\mathrm{H}_{2}$ and $\mathrm{O}_{2}$ from water and thereafter, review recent advancements in the catalytic performances of electrocatalysts towards the overall water splitting.

\subsection{Electrochemistry of water splitting}

A typical water splitting electrolytic cell was first proposed in 1789 by J. R. Deiman and Adriaan Paets van Troostwijk. ${ }^{26}$ The water splitting process involves two half-cell reactions: anodic oxidation and cathodic reduction reactions. Alternatively, these reactions are conceptualised as oxygen evolution reaction (OER) and hydrogen evolution reaction (HER), respectively.

Based on the nature of the electrolytes, the specific half-cell reactions are different at the electrodes. Nevertheless, the overall reaction is the same. ${ }^{6,27}$

In an acidic electrolyte:

At the cathode:

$$
2 \mathrm{H}^{+}+2 \mathrm{e}^{-} \rightarrow 2 \mathrm{H}_{2}+\mathrm{O}_{2}, \quad E_{\mathrm{c}}=0 \mathrm{~V}
$$

At the anode:

$$
2 \mathrm{H}_{2} \mathrm{O} \rightarrow \mathrm{O}_{2}+4 \mathrm{H}^{+}+4 \mathrm{e}^{-}, \quad E_{\mathrm{a}}=1.23 \mathrm{~V}
$$

In an alkaline electrolyte:

At the cathode:

$$
2 \mathrm{H}_{2} \mathrm{O}+2 \mathrm{e}^{-} \rightarrow \mathrm{H}_{2}+2 \mathrm{OH}^{-}, \quad E_{\mathrm{c}}=-0.83 \mathrm{~V}
$$

At the anode:

$$
4 \mathrm{OH}^{-} \rightarrow \mathrm{O}_{2}+2 \mathrm{H}_{2} \mathrm{O}+4 \mathrm{e}^{-}, \quad E_{\mathrm{a}}=-0.40 \mathrm{~V}
$$

Overall reaction:

$$
2 \mathrm{H}_{2} \mathrm{O} \rightarrow 2 \mathrm{H}_{2}+\mathrm{O}_{2}
$$

Regardless of the electrolytes, the theoretical reaction potential difference of the process is $1.23 \mathrm{~V}$ at $25{ }^{\circ} \mathrm{C}$ and atmospheric pressure. The cell potential of the cathode $\left(E_{\mathrm{c}}\right)$ and anode $\left(E_{\mathrm{a}}\right)$ is based on the normal hydrogen electrode (NHE). For practical application of the water splitting process, an excess potential (overpotential, $\eta_{\mathrm{T}}$ ) is employed for reporting. This excess potential is primarily to overcome the inherent activation difficulties at the cathode $\left(\eta_{\mathrm{c}}\right)$ and anode $\left(\eta_{\mathrm{a}}\right)$ and other associated hindrances $\left(\eta_{\text {other }}\right)$ like contact resistance. Realistically, the overall 
water splitting reaction is represented with an operational potential $\left(E_{\mathrm{OP}}\right)$ defined by

$$
\begin{gathered}
E_{\mathrm{OP}}=1.23 \mathrm{~V}+\eta_{\mathrm{T}} \\
E_{\mathrm{OP}}=1.23 \mathrm{~V}+\eta_{\mathrm{a}}+\eta_{\mathrm{c}}+\eta_{\text {other }}
\end{gathered}
$$

From this equation, it is paramount to decrease the overpotential $\left(\eta_{\mathrm{T}}\right)$ for the overall reaction to be energy-efficient and economical. Undeniably, the effective reduction of $\eta_{\mathrm{c}}$ and $\eta_{\mathrm{a}}$ could be achieved by HER and OER electrocatalysts, respectively. In line with this, optimizing the design of the electrolyzer is an effective strategy for minimizing $\eta_{\text {other }}$. Detailed discussion on the overpotential and measures for its reduction are described in the literature. ${ }^{26}$ To ensure that the overall water splitting process is more economical, the understanding of the fundamentals with recent theoretical insight and the development of efficient HER and OER electrocatalysts are essential. In addition, studies should preferably be centred on non-hazardous, earth-abundant and less expensive materials. ${ }^{28}$

2.1.1. Fundamentals of hydrogen evolution reaction (HER). HER involves a multi-step process with the transfer of twoelectrons at the surface of the cathode by two different mechanisms via three probable reactions. ${ }^{29,30}$

In an acidic medium ${ }^{30,31}{ }^{*}$ indicates the active site on the catalyst surface),

$$
\begin{gathered}
\mathrm{H}_{3} \mathrm{O}^{+}+\mathrm{e}^{-}+{ }^{*} \rightarrow \mathrm{H}^{*}+\mathrm{H}_{2} \mathrm{O}, \quad \text { Volmer reaction } \\
\mathrm{H}^{*}+\mathrm{H}_{3} \mathrm{O}^{+}+\mathrm{e}^{-} \rightarrow \mathrm{H}_{2}+\mathrm{H}_{2}, \quad \text { Heyrovsky reaction } \\
\mathrm{H}^{*}+\mathrm{H}^{*} \rightarrow \mathrm{H}_{2}, \quad \text { Tafel reaction }
\end{gathered}
$$

However, in an alkaline medium, ${ }^{29}$

$$
\begin{array}{r}
\mathrm{H}_{2} \mathrm{O}^{+}+\mathrm{e}^{-}+{ }^{*} \rightarrow \mathrm{H}^{*}+\mathrm{OH}^{-}, \text {Volmer reaction } \\
\mathrm{H}^{*}+\mathrm{H}_{2} \mathrm{O}+\mathrm{e}^{-} \rightarrow \mathrm{H}_{2}+\mathrm{OH}^{-}, \quad \text { Heyrovsky reaction } \\
\mathrm{H}^{*}+\mathrm{H}^{*} \rightarrow \mathrm{H}_{2}, \quad \text { Tafel reaction }
\end{array}
$$

Generally, HER in an alkaline medium is relatively slower than in the acidic medium as it involves the prior dissociation of $\mathrm{H}_{2} \mathrm{O}$ to yield the $\mathrm{H}^{*}$ intermediates. Irrespective of the HER pathway, the formation of the $\mathrm{H}^{*}$ intermediate is necessary. Thus, estimating the extent of the $\mathrm{H}^{*}$ adsorption process provides a critical clue about the favorability of the electrode surface to undergo HER. Following this fact, the free energy of hydrogen adsorption $\left(\Delta G_{\mathrm{H}^{*}}\right)$ is a universally established descriptor for hydrogen-evolving materials. The $\Delta G_{\mathrm{H}^{*}}$ value for an optimal HER catalyst is estimated to be close to zero. The reason for this is that a large $\Delta G_{\mathrm{H}^{*}}$ suggests the difficulty of breaking the adsorbed $\mathrm{H}^{*}$ and hence, impeding $\mathrm{H}_{2}$ desorption. Moreover, a low $\Delta G_{\mathrm{H}^{*}}$ value indicates a weak $\mathrm{H}^{*}$ adsorption resulting in a poor interaction between the cathode and the protons.

A computationally derived volcano plot of theoretical $\Delta G_{\mathrm{H}^{*}}$ $v s$. the exchange current densities $\left(\log j_{0}\right)$ reflects the HER activities for a range of catalysts. ${ }^{32}$ This plot proposes an instinctive approach to visualize and compare the activity of a range of catalysts to enable the optimization of material design for HER. In addition, understanding the relative HER mechanism for each material is also crucial towards its design. The Tafel slope, derived from the Tafel plot and polarization curve, can be used to understand the HER mechanism. ${ }^{33,34}$ This slope is an inherent property of the catalyst, which is computed to define the rate-determining step of the HER process. In general, there are three Tafel slopes recognized to get insight into the reaction kinetics in HER, namely, $29 \mathrm{mV} \mathrm{dec}^{-1}, 39 \mathrm{mV} \mathrm{dec}^{-1}$ and $118 \mathrm{mV} \mathrm{dec}^{-1}$, representing the Tafel, Heyrovsky and Volmer reactions, respectively. The rate-determining step of an electrode is defined by the proximity of its Tafel slope to that of the above-mentioned reactions. However, it is a challenge to unequivocally distinguish whether the rate-determining step is Tafel and Heyrovsky given that the values of their Tafel slopes are similar.

For instance, Zhao and co-workers reported that the Tafel slope of a Pt electrode with the $\mathrm{Pt}(110)$ plane is $\sim 30 \mathrm{mV} \mathrm{dec}^{-1}$; however, the rate-determining step could be either the Tafel or Heyrovsky reaction despite the value being close to that of Tafel reaction..$^{35}$ To distinguish these reactions, the rate-determining steps (Tafel or Heyrovsky) should be related to the surface coverage of $\mathrm{H}^{*}$ on the electrode..$^{35,36}$ The determination and interpretation of the reaction mechanisms are significant to gain theoretical insight into the elementary steps involved in HER.

2.1.2. Fundamentals of oxygen evolution reaction (OER). As mentioned earlier, OER occurs at the anode as a multi-step process involving the transfer of four-electrons. The mechanism and reaction routes for OER are relatively more intricate than that for HER owing to the slow electro-kinetic profile. ${ }^{37}$ Similar to HER, OER differs depending on the medium. In an acidic medium, oxygen $\left(\mathrm{O}_{2}\right)$ and a hydrogen ion are formed from the oxidation of $\mathrm{H}_{2} \mathrm{O}$, whereas in an alkaline or neutral medium, $\mathrm{H}_{2} \mathrm{O}$ and $\mathrm{O}_{2}$ are formed from the oxidation of a hydroxyl ion $\left(\mathrm{OH}^{-}\right)$.

These reactions occur with the involvement of adsorbed $\mathrm{OH}^{-}, \mathrm{O}^{2-}$ and $\mathrm{OOH}^{-}$intermediates on the surface of the catalyst. Hypothetically, a generally accepted OER mechanism is illustrated as follows. ${ }^{38}$

OER in an acidic medium:

$$
\begin{aligned}
\mathrm{H}_{2} \mathrm{O}+{ }^{*} & \rightarrow \mathrm{HO}^{*}+\mathrm{H}^{+}+\mathrm{e}^{-} \\
\mathrm{HO}^{*} & \rightarrow \mathrm{O}^{*}+\mathrm{H}^{+}+\mathrm{e}^{-} \\
\mathrm{O}^{*}+\mathrm{H}_{2} \mathrm{O} & \rightarrow \mathrm{HOO}^{*}+\mathrm{H}^{+}+\mathrm{e}^{-} \\
\mathrm{HOO}^{*} & \rightarrow{ }^{*}+\mathrm{O}_{2}+\mathrm{H}^{+}+\mathrm{e}^{-}
\end{aligned}
$$

OER in an alkaline medium:

$$
\begin{gathered}
\mathrm{OH}^{-}+{ }^{*} \rightarrow \mathrm{HO}^{*}+\mathrm{e}^{-} \\
\mathrm{OH}^{-}+\mathrm{HO}^{*} \rightarrow \mathrm{O}^{*}+\mathrm{H}_{2} \mathrm{O}+\mathrm{e}^{-} \\
\mathrm{O}^{*}+\mathrm{OH}^{-} \rightarrow \mathrm{HOO}^{*}+\mathrm{e}^{-} \\
4 \mathrm{HOO}^{*} \rightarrow{ }^{*}+\mathrm{O}_{2}+2 \mathrm{H}_{2} \mathrm{O}+\mathrm{e}^{-}
\end{gathered}
$$


Recently, density functional theory (DFT) computation provided insight into the underlying mechanisms for each reaction step as follows: ${ }^{39}$

$$
\begin{array}{r}
2 \mathrm{H}_{2} \mathrm{O}+{ }^{*} \rightarrow \mathrm{H}_{2} \mathrm{O}+\mathrm{HO}^{*}+\mathrm{H}^{+}+\mathrm{e}^{-} \\
\Delta G_{1}=\Delta G_{\mathrm{HO}^{*}}-\Delta G_{\mathrm{H}_{2} \mathrm{O}}-\mathrm{e} U+k_{\mathrm{B}} T \ln \left[\mathrm{H}^{+}\right] \\
\mathrm{H}_{2} \mathrm{O}+\mathrm{HO}^{*} \rightarrow \mathrm{H}_{2} \mathrm{O}+\mathrm{O}^{*}+\mathrm{H}^{+}+\mathrm{e}^{-} \\
\Delta G_{2}=\Delta G_{\mathrm{O}^{*}}-\Delta G_{\mathrm{HO}^{*}}-\mathrm{e} U+k_{\mathrm{B}} T \ln \left[\mathrm{H}^{+}\right] \\
\mathrm{O}^{*}+\mathrm{H}_{2} \mathrm{O} \rightarrow \mathrm{HOO}^{*}+\mathrm{H}^{+}+\mathrm{e}^{-} \\
\Delta G_{3}=\Delta G_{\mathrm{HOO}^{*}}-\Delta G_{\mathrm{O}^{*}}-\mathrm{e} U+k_{\mathrm{B}} T \ln \left[\mathrm{H}^{+}\right] \\
\mathrm{HOO}^{*} \rightarrow{ }^{*}+\mathrm{O}_{2}+\mathrm{H}^{+}+\mathrm{e}^{-} \\
\Delta G_{4}=\Delta G_{\mathrm{O}_{2}}-\Delta G_{\mathrm{HOO}^{*}}-\mathrm{e} U+k_{\mathrm{B}} T \ln \left[\mathrm{H}^{+}\right]
\end{array}
$$

where $\Delta G_{i}$ is the Gibbs free energy of the $i$ th reaction step; $U$ represents the measured electrode potential ( $v s$. NHE under standard conditions); $k_{\mathrm{B}}$ is the Boltzmann constant; and $T$ is the absolute temperature. $\Delta G_{1}, \Delta G_{2}$, and $\Delta G_{3}$ (and $\Delta G_{4}$ ) refer to the adsorption energies of $\mathrm{HO}^{*}, \mathrm{O}^{*}$ and $\mathrm{HOO}^{*}$, respectively. For an ideal case, $\Delta G_{\mathrm{HO}^{*}}, \Delta G_{\mathrm{O}^{*}}$, and $\Delta G_{\mathrm{HOO}^{*}}$ are $1.23 \mathrm{eV}, 2.46 \mathrm{eV}$ and $3.69 \mathrm{eV}$, respectively. ${ }^{40}$

Under standard conditions, when the measured electrode potential is 0 versus the standard hydrogen electrode (SHE), the theoretical overpotential $\eta^{\text {OER }}$ is defined as ${ }^{26}$

$$
\eta^{\mathrm{OER}}=\left(\frac{G^{\mathrm{OER}}}{e}\right)-1.23 \mathrm{~V}
$$

where $G^{\mathrm{OER}}$ is the largest Gibbs free energy of the reactions $\left(G^{\mathrm{OER}}=\max \left\{\Delta G_{1}, \Delta G_{2}, \Delta G_{3}, \Delta G_{4}\right\}\right){ }^{41}$

For minimal overpotential ( $\eta^{\mathrm{OER}}=0$ ), $\Delta G^{\mathrm{OER}}$ is $1.23 \mathrm{eV}$. Given that $\Delta G_{1}=\Delta G_{2}=\Delta G_{3}=\Delta G_{4}$ for an ideal OER catalyst under standard conditions, this suggests that the $\Delta G$ for each step at $\eta^{\text {OER }}=1.23 \mathrm{eV}^{40}{ }^{40}$ Based on this hypothesis, a volcano plot analogous to that for the HER catalysts is obtained for a range of OER catalysts (Fig. 1). Here, the standard free energy $\left(\Delta G_{\mathrm{O}^{*}}^{0}-\Delta G_{\mathrm{HO}^{*}}^{0}\right)$ is plotted against the theoretical overpotential to reflect the OER activities for a series of catalysts. $\Delta G_{\mathrm{O}^{*}}^{0}-$ $\Delta G_{\mathrm{HO}^{*}}^{0}$ is a notable descriptor for OER activity. Deductions from the volcano plot indicate that the plot can be beneficial towards the design and optimization of highly efficient OER catalysts. Moreover, the Tafel slope, which is also correlated with the OER overpotential, can direct the understanding of the OER mechanism by providing kinetic information of OER catalysts. ${ }^{42}$

\subsection{Electrocatalysts for water splitting}

To sustain an efficient and economic water splitting process, HER-OER bifunctional catalysts that can offer a minimum overpotential in aqueous alkaline or acidic medium are indispensable. ${ }^{44}$ However, the design and development of electrocatalysts are complex and challenging as several factors need to be considered in this regard. ${ }^{45}$ For instance, the electrocatalysts suitable for alkaline solutions may be unstable or inactive in acidic medium, and vice versa. Therefore, it is

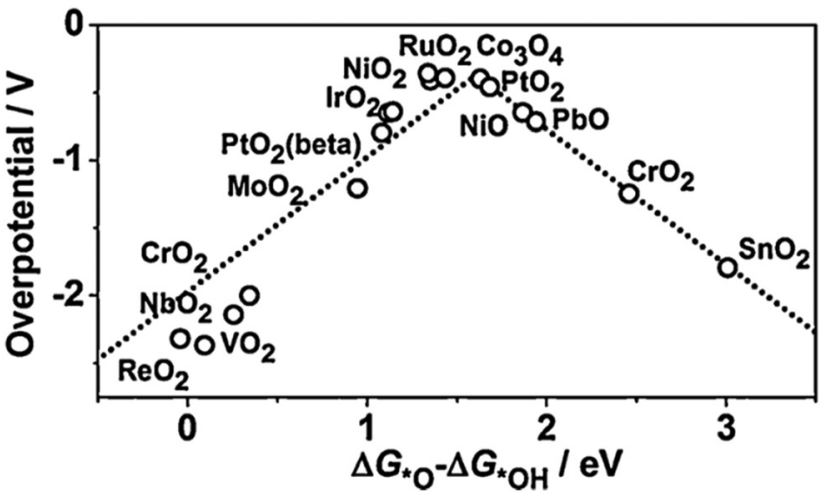

Fig. 1 Volcano plot for OER on metal oxides. Reproduced with permission. ${ }^{43}$ Copyright 2015, Elsevier.

exceedingly attractive to develop bifunctional electrocatalysts with high activity towards both OER and HER in the same electrolyte. $^{46,47}$

Hitherto, both metal-based and metal-free catalyst systems have shown potential to catalyze the water splitting process. Metal-based catalyst systems including noble metals (such as iridium (Ir), palladium (Pd), platinum (Pt), ruthenium $(\mathrm{Ru})$ and gold ( $\mathrm{Au})$ ) and transition metals (TMs, such as cobalt (Co), iron $(\mathrm{Fe})$, nickel (Ni), molybdenum (Mo) and copper $(\mathrm{Cu})$ ) and their compounds are the most widely utilized HER-OER electrocatalysts. However, owing to their structural complexities, physiochemical challenges and thermodynamic instability, these catalyst systems are constrained by their weak durability and low selectivity. This coupled with their high cost has led to research endeavor to seek for alternatives to replace metalbased electrocatalysts for water splitting. ${ }^{44}$

Following the discovery of carbon-based materials as oxygen reduction reaction (ORR) catalysts in 2009 , steps were taken to design and develop metal-free electrocatalysts for water splitting. Thereafter, considerable headway has been made in this regard, particularly with respect to earth-abundant metal-free materials. ${ }^{44,45}$ Beyond their natural abundance, metal-free electrocatalysts have demonstrated good catalytic performance for water splitting due to their remarkable electrical conductivity, large surface area and high tolerance under wide operating conditions. ${ }^{46,48}$ Moreover, the flexible architecture of metal-free catalysts exposes these materials to potential heteroatom doping and structure reengineering, which can modulate the charge distribution of the carbon genomics with potential synergistic effects for water splitting. ${ }^{49}$ Notable examples of these catalysts include heteroatom-doped carbon nanotubes and graphene. ${ }^{50}$

Apart from the category, composition and structure of electrocatalysts, the properties (e.g. $\mathrm{pH})$ of electrolytes play a role in the electrochemical water splitting reactions. The acidic electrolytes are beneficial for water splitting as they offer more hydroniums $\left(\mathrm{H}_{3} \mathrm{O}^{+}\right)$with weak covalent bonding and thereby lead to fast reaction kinetics for the reduction reaction at the cathode while the alkaline water splitting is more popular than acidic water splitting because of the availability of more choice 
of electrocatalysts for anodic half-cell OER reaction and the facile formation of $\mathrm{OH}^{*}$ and $\mathrm{O}^{*}$ species. ${ }^{51}$ Moreover, the strong acid condition requires the employment of an acid proton exchange membrane and suffers from the issue of high cost and pollution from the evaporated acid electrolyte. Comparatively, an alkaline electrolyte can relieve these problems in acid conditions for water splitting. The alkaline environment, typically realized by the adoption of $1.0 \mathrm{M} \mathrm{KOH}$ solution, has been widely employed for water splitting and has demonstrated inspiring performance over the past few decades. ${ }^{51}$ However, the drawbacks of harsh alkaline or acidic electrolytes such as the corrosion issue and the requirement of specific ion exchange membranes with good stability are inescapable when it comes to applications. Therefore, electrochemical water splitting under neutral and near-neutral conditions has gained much research interest. ${ }^{52,53}$ Different from water electrolysis under extreme acidic or alkaline conditions, water splitting under neutral or near-neutral conditions is seriously affected by reactant switching and identity and concentration of the buffered anions in similar $\mathrm{pH}$ values. Thus, buffer solutions, for instance phosphate buffered saline $(\mathrm{pH} 7)$, bicarbonate and carbonate buffer solution ( $\mathrm{pH} 9.2$ to 10.6), sodium sulfate solution ( $\mathrm{pH} 7$ ) and borate buffer solution ( $\mathrm{pH} 8.5$ ), are widely used in neutral electrocatalytic water splitting. ${ }^{54}$

Shu-Hong $\mathrm{Yu}$ and co-workers ${ }^{55}$ reported the synthesis and application of ternary $\mathrm{Ni}_{0.1} \mathrm{Co}_{0.9} \mathrm{P}$ porous nanosheets onto conductive carbon fiber paper which exhibited promising potential in neutral-pH water electrolysis. The ternary $\mathrm{Ni}_{0.1} \mathrm{Co}_{0.9} \mathrm{P}$ catalyst endows the resultant device with a voltage requirement of mere $1.81 \mathrm{~V}$ to reach a current density of $10 \mathrm{~mA} \mathrm{~cm}^{-2}$, showing excellent efficiency among the noble-metal-free neutral-pH electrolyzers. Jie $\mathrm{Yu}$ and co-workers ${ }^{56}$ reviewed the recent research on the electrocatalytic water-splitting performance of precious-metal-free catalytic materials in neutral media. Anantharaj and Aravindan ${ }^{54}$ summarized 3d transition-metalbased electrocatalysts for neutral and near-neutral water splitting from the perspective of activity, selectivity, and stability. These reviews provide a comprehensive summary of the catalytic performance of catalysts with neutral electrolytes. It is worth noting that the neutral electrolytes are good electrolytes with potential as they have more similar physical and chemical properties to seawater. However, most electrocatalysts towards overall water splitting showed better efficiency in alkaline electrolytes. It is still promising to develop efficient electrocatalysts in a neutral or near neutral pH environment.

In general, bifunctional electrocatalysts preferentially favor either the HER or OER depending on their intrinsic characteristics. Hence, to enable a high overall performance, the intrinsic activity and physiochemical properties of the catalysts should be synergistically improved. ${ }^{46}$ In this section, we critically review the recent advances in metal-based and metal-free electrocatalysts towards the overall water splitting process, and discuss the theoretical perspectives in understanding the catalytic process and recent challenges.

2.2.1 Noble metal-based electrocatalysts. The design and development of an energy-efficient electrocatalyst for cathodic
HER and anodic OER are aimed to overcome the large watersplitting overpotentials. ${ }^{20}$ To date, the noble metal-based electrocatalysts (Ir, Pd, Pt, $\mathrm{Rh}$ and $\mathrm{Ru}$ ) are still the most effective catalyst systems for water splitting, particularly in an acidic solution. Pt-based materials are known to possess the highest activity toward HER, ${ }^{57}$ while Ir- and Ru-based materials are the most efficient OER electrocatalysts. ${ }^{58}$ This is due to their superior properties such as large specific surface area, unique pore structure, and excellent structural stability. ${ }^{59,60}$ However, owing to their rarity and high cost, research studies have engaged in developing bifunctional electrocatalysts with minimal noble metal loading, offering high catalytic activity and stability. Numerous schemes such as size reduction, shape modulation, heteroatom doping, and anchoring noble metals on a support have been proposed to improve the performances. ${ }^{60}$ The alloying of noble metals with bi- or multi-metallic elements has been proven to improve the OER-HER activity of noble metals. ${ }^{61}$ Guo and co-workers demonstrated the superior water splitting performance of bimetallic doped IrCoNi porous hollow nanocrystals (PHNCs) over the single doping. When tested in $0.5 \mathrm{M}$ $\mathrm{H}_{2} \mathrm{SO}_{4}$, IrCoNi PHNCs displayed a low cell voltage of $1.56 \mathrm{~V}$ at $2 \mathrm{~mA} \mathrm{~cm} \mathrm{~cm}^{-2}$ current density with excellent stability after 1000 cycles (Fig. 2(a) and (b)). ${ }^{61}$ The improved electrocatalytic activity was attributed to the large surface area due to the porous nature and size reduction.

Alternatively, the anchoring of noble metals on support surfaces has presented a myriad of novel heterogeneous electrocatalysts with high HER-OER activity. This approach typically augments the interaction between the metals and the support, thereby leading to an enhanced charge distribution. Specifically, exploiting surface defects on the support (particularly supports that offer enriched coordinating atoms with lone electron pairs as active centers) and fabricating porous frameworks are the most recently adopted schemes to improve the catalytic activity. ${ }^{60}$ For instance, Lee and co-workers utilized a structure-supporting hemispherical core-shell to fortify the HER-OER activity on an Ir-based multi-metallic nanoframe anchored on an Au-based core (AuCu@IrNi). ${ }^{62}$ Here, the authors demonstrated that the developed hemicore@frame AuCu@IrNi complex suitably catalyzed OER and HER in $0.1 \mathrm{M} \mathrm{HClO}_{4}$ with low overpotentials of $308 \mathrm{mV}$ and $13.7 \mathrm{mV}$ with Tafel slopes of $58 \mathrm{mV} \mathrm{dec}{ }^{-1}$ and $22 \mathrm{mV} \mathrm{dec}^{-1}$, respectively (Fig. 2(c) and (d)).

Table 1 summarizes the catalytic activity of some of the bifunctional water splitting electrocatalysts. Generally, most noble metals are inclined to favor either the OER or HER process; therefore they are not so effective for bifunctional water splitting, particularly in the same medium. It is well documented that modulating the surface morphology, electronic structure, and element doping (bi- or multi-metallic) plays a crucial role in upgrading the activity of noble metal-based materials as OER-HER bifunctional electrocatalysts. Nonetheless, the commercial application of these catalysts is limited by their scarcity and high cost. This has driven the research interest for the design of efficient catalyst systems made from readily available and low-cost materials such as earth-abundant materials. 

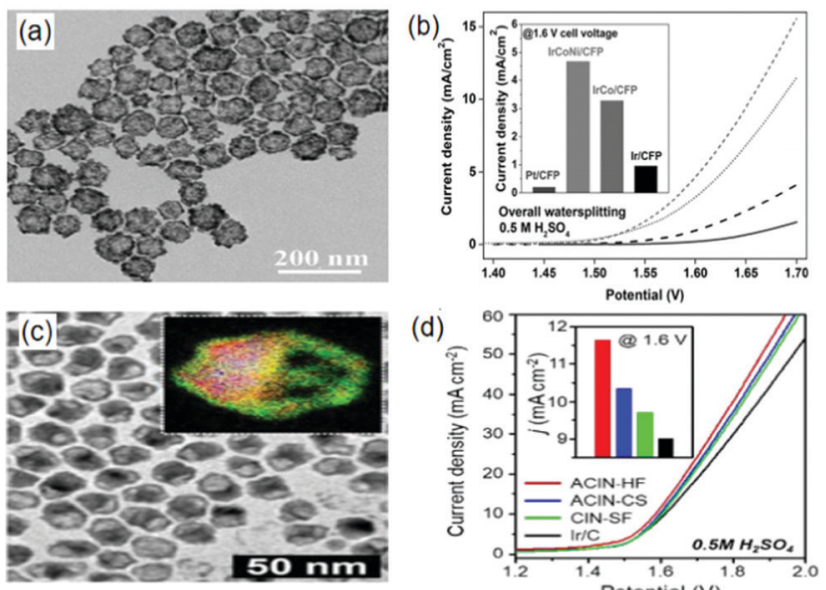

(d)

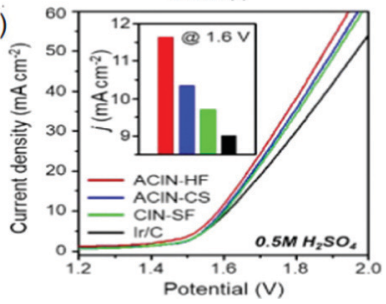

Fig. 2 (a) TEM image of IrCoNi PHNCs and their (b) polarization curve in comparison to that of IrCo/CFP, Ir/CFP, and Pt/CFP for overall water splitting in $0.5 \mathrm{M} \mathrm{H}_{2} \mathrm{SO}_{4}$ solution at a scan rate of $5 \mathrm{mV} \mathrm{s}^{-1}$ (inset: with the corresponding current densities at $1.6 \mathrm{~V}$ ). Panels (a) and (b) are reproduced with permission. ${ }^{61}$ Copyright 2017, Wiley-VCH. (c) TEM image of AuCu@IrNi and (d) polarization curves of ACIN-HF/CFP, ACIN-CS/CFP, CIN-SF/CFP and Ir/CFP for overall water splitting in $0.1 \mathrm{M} \mathrm{HClO}_{4}$ solution at a scan rate of $5 \mathrm{mV} \mathrm{s}^{-1}$ (inset: with the corresponding current densities). Panels (c) and (d) are reproduced with permission. ${ }^{62}$ Copyright 2019, Royal Society of Chemistry.

2.2.2 Non-noble metal-based electrocatalysts. Recently, a vast number of non-noble metal-based electrocatalysts have been extensively investigated for electrochemical water splitting. ${ }^{15}$ Among them, ${ }^{60,63,64}$ this section will focus on earth-abundant transition metal-based electrocatalysts (such as Ni-, Co-, Cu-, Fe-, Mo- and W-based materials) due to their theoretically asserted high electrocatalytic performances with low-cost. ${ }^{65,66}$ To present a fair comparison, the different electrocatalysts have been divided into major groups including metallic substances, metal hydroxides/oxides, metal chalcogenides, metal phosphates/phosphides, metal nitrides and metal carbides. Correspondingly, the investigated bifunctional electrocatalysts are listed in Table 1.

Metallic substance. Metallic substances are well-known electrocatalysts that are commonly used in the commercial application of electrolytic water splitting. ${ }^{67,68}$ Nevertheless, this set of electrocatalysts are known to exhibit low efficiency and less stability necessitating measures to enhance their electrocatalytic activity. For instance, Martindale and Reisner reported the unstable condition of Fe due to its dissolution at the anodic and cathodic potentials under neutral and acidic media, respectively. ${ }^{69} \mathrm{Simi}$ larly, Jahan and co-workers reported the instability of $\mathrm{Cu}$ complexes due to their corrosion and instability in an acidic medium. ${ }^{63}$

One option is incorporating the metal based materials with non-metal or another transition metal based dopant. ${ }^{68,70-72}$ Our group reported an amorphous Ni-B nanoparticle film on $\mathrm{Ni}$ foam (Ni-B/Ni foam) with good water splitting potential (Fig. 3(a) and (b)). An electrolytic cell voltage of $1.69 \mathrm{~V}$ was required to attain $15 \mathrm{~mA} \mathrm{~cm}{ }^{-2}$ current density in $1.0 \mathrm{M} \mathrm{KOH}$ with overpotentials of $125 \mathrm{mV}$ and $360 \mathrm{mV}$ at $20 \mathrm{~mA} \mathrm{~cm} \mathrm{~cm}^{-2}$ and $100 \mathrm{~mA} \mathrm{~cm}{ }^{-2}$ for the HER and OER, respectively. ${ }^{68}$
In the past, $\mathrm{Co}^{70,73-75}$ and Fe-based ${ }^{76-78}$ compounds have been used as potential water splitting electrocatalysts. However, given that $\mathrm{Fe}$ is one of the most abundant earth metals and highly attractive for the development of low-cost catalysts, Fe-based compounds have been extensively examined as HER or OER electrocatalysts. ${ }^{76-78}$ Nonetheless, similar to Co-based metallic compounds, only a fair number of studies have reported the bifunctionally active catalysts compared to the Fe-based metallic compounds for water splitting. Among those studies, Martindale and Reisner demonstrated an Fe-only electrode to be active for catalysing both proton reduction and water oxidation in alkaline medium with superior activity than bifunctional $\mathrm{Co}$ and Ni electrocatalysts. ${ }^{69}$ The authors also demonstrated that the electrolyzer system with an Fe-only electrode was relatively more stable and durable. This was ascribed to the reversible interconversion of catalytically active Fe-species (an iron oxide-hydroxide $\left(\mathrm{FeO}_{x}\right)$ phase under the anodic bias and $\mathrm{Fe}(0)$ phase under the cathodic bias).

Another example of low-cost catalytic materials is $\mathrm{Cu}$ and its derivatives, which are reported to be beneficial towards the activation of oxygen reduction reaction $(\mathrm{ORR})^{79}$ and serve as HER/OER electrocatalysts. ${ }^{63,80}$ However, similar to Co-based metallic materials, the practical application of $\mathrm{Cu}$-based metallic catalysts is hindered by their large overpotential and/or low stability, which requires further modifications. For instance, Jahan and co-workers fabricated a Cu-MOF composite by anchoring a Cu-centered MOF on graphene oxide (GO), ${ }^{63}$ which was employed as a tri-functional HER, OER and ORR electrocatalyst, particularly in an acidic solution. The exceptional performance of the catalyst was founded on the GO-MOF synergistic effects including the unique porous scaffold structure and improved electron transport. Likewise, anchoring Co on $\mathrm{N}$-doped carbon nanostructures $(\mathrm{Co}-\mathrm{N}-\mathrm{C})^{81,82}$ has been demonstrated to result in robust water splitting electrocatalysts due to the synergistic chemical coupling between the embedded Co and the $\mathrm{N}$-dopant or carbon layers, leading to a better H-bonding energy necessary for $\mathrm{HER}^{82}$ and both greater mechanical and chemical rigidity.

Furthermore, the doping of these metallic catalysts with other metals or non-metals has also been shown to improve their individual electrocatalytic performance. For instance, the incorporation of $\mathrm{Fe}$ with $\mathrm{Ni}$ resulted in a $\mathrm{Ni}-\mathrm{Fe}$ composite which displayed high potential as a HER-OER electrocatalyst in alkaline solutions, requiring overpotentials of only 240 and $270 \mathrm{mV}$ to deliver current densities of 500 and $1000 \mathrm{~mA} \mathrm{~cm}^{-2}$, respectively. The electrode also displayed prolonged stability against bulk water electrolysis at large currents ${ }^{83}$ attributed to the optimal behaviour of this binary film due to the stabilizing effect of Fe on $\mathrm{Ni}$ at a higher oxidation level. ${ }^{84}$

The use of 3D catalytic substrates has been demonstrated towards improving the surface morphology, exposed active centres and electrical characteristics of the electrocatalysts. ${ }^{70,83,85,86}$ A commonly used low-cost conductive substrate is Ni foam, which when compared to other substrates such as $\mathrm{Ni}$ foil has shown superior activity due to its $3 \mathrm{D}$ macroporous structure and structure-induced electronic effect. ${ }^{83}$ In addition, Ni foam 
Table 1 Summary of the performances of water-splitting electrocatalysts

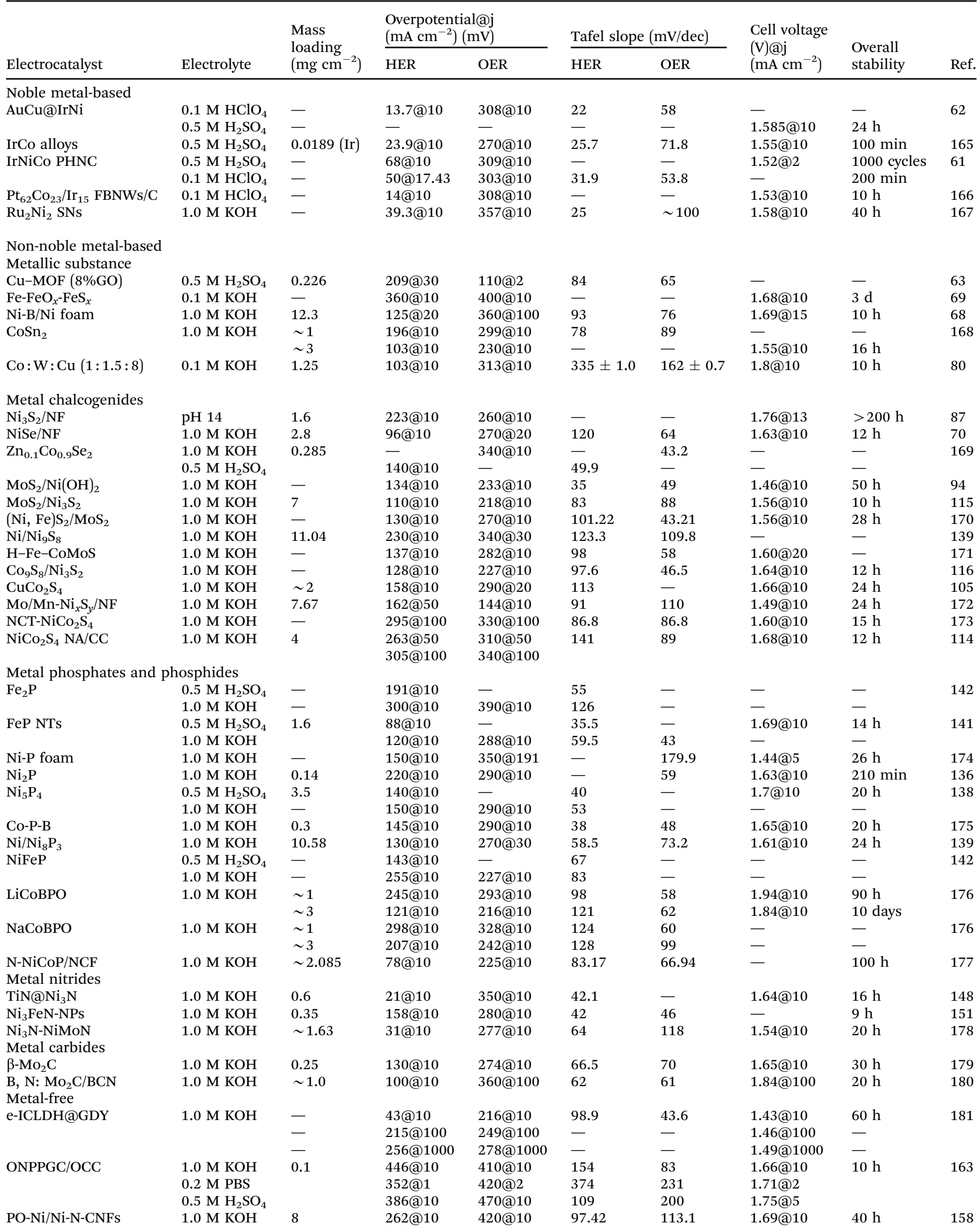



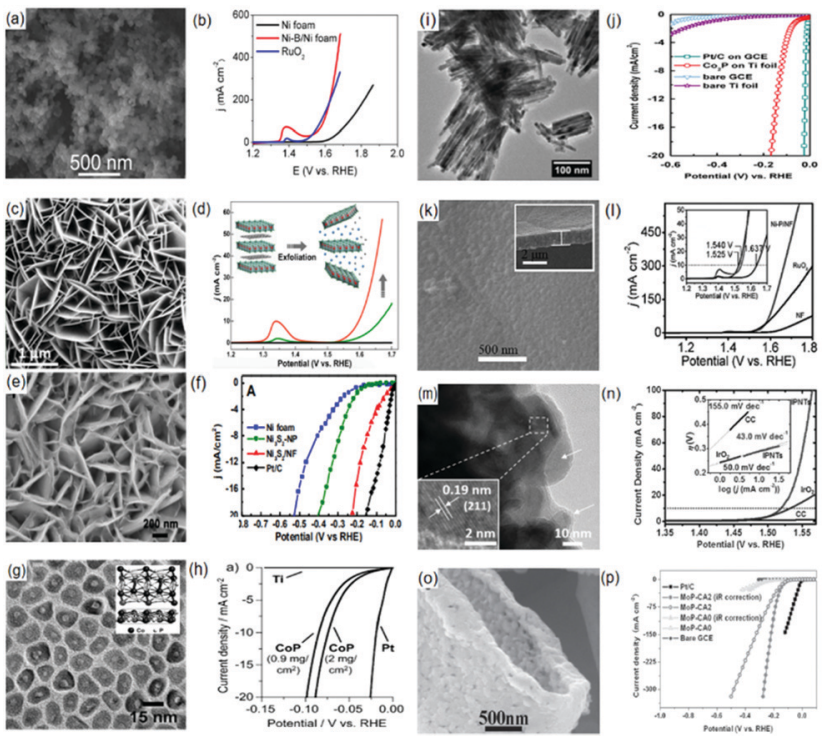

Fig. 3 (a) SEM image of $\mathrm{Ni}-\mathrm{B} / \mathrm{Ni}$ foam. Polarization curve (in $1.0 \mathrm{M} \mathrm{KOH}$ at a scan rate of $2 \mathrm{mV} \mathrm{s}^{-1}$ ) for (b) OER on Ni foam, Ni-B/Ni foam and $\mathrm{RuO}_{2}-\mathrm{Ni}$ foam. Panels (a) and (b) are reproduced with permission. ${ }^{68}$ Copyright 2016 , IOP Publishing Ltd. (c) TEM image of as-synthesized NiCo LDH nanoplates on carbon paper via HCFR. (d) Polarization curves (in $\mathrm{O}_{2}$-saturated $1 \mathrm{M}$ $\mathrm{KOH}$ at a scan rate of $0.5 \mathrm{mV} \mathrm{s}^{-1}$ ) of NiCo LDH catalysts and carbon paper Images (c) and (d) are reproduced with permission. ${ }^{113}$ Copyright 2015, American Chemical Society. (e) Top-view SEM image of $\mathrm{Ni}_{3} \mathrm{~S}_{2} / \mathrm{NF}$. (f) Steady-state current density as a function of applied voltage during HER at $\mathrm{pH} 7$ over nickel foam (NF), $\mathrm{Ni}_{3} \mathrm{~S}_{2}-\mathrm{NP}, \mathrm{Ni}_{3} \mathrm{~S}_{2} / \mathrm{NF}$ and $\mathrm{Pt} / \mathrm{C}(20$ wt\%). Images (e) and (f) are reproduced with permission. ${ }^{87}$ Copyright 2015, American Chemical Society. (g) TEM image of CoP nanoparticles. (h) HER performance of the $\mathrm{CoP} / \mathrm{Ti}$ electrode in $0.5 \mathrm{M} \mathrm{H}_{2} \mathrm{SO}_{4}$. Panels (g) and (h) are reproduced with permission. ${ }^{129}$ Copyright 2014, Wiley (i) TEM image of the $\mathrm{CO}_{2} \mathrm{P}$ nanorods. (j) Polarization curves of $\mathrm{Pt} / \mathrm{C}$ on GCE (loading amount: $0.285 \mathrm{~g} \mathrm{~cm}^{-2}$ ), $\mathrm{CO}_{2} \mathrm{P}$ on Ti foil, bare GCE, and bare Ti foil in $0.5 \mathrm{M} \mathrm{H}_{2} \mathrm{SO}_{4}$ solution (iR corrected). The bare Ti foil was subjected to the same annealing treatment as $\mathrm{CO}_{2} \mathrm{P}$ on a $\mathrm{Ti}$ foil sample prior to the measurement. Panels (i) and (j) are reproduced with permission. ${ }^{130}$ Copyright 2014, Elsevier. (k) SEM image of Ni-P/NF (inset: cross-sectional analysis). (l) Polarization curve (in $1.0 \mathrm{M} \mathrm{KOH}$ at a scan rate of $2 \mathrm{mV} \mathrm{s}^{-1}$ ) for OER on $\mathrm{Ni}$ $\mathrm{P} / \mathrm{NF}, \mathrm{NF}$, and Pt/C-NF. Panels $(\mathrm{k})$ and $(\mathrm{l})$ are reproduced with permission. ${ }^{85}$ Copyright 2015, Wiley. (m) HRTEM image of IPNTs (inset: enlarged HRTEM image). (n) iR-Corrected LSV curves measured in $1.0 \mathrm{M} \mathrm{KOH}$ (inset: the corresponding Tafel slopes). Panels $(\mathrm{m})$ and $(\mathrm{n})$ are reproduced with permission. ${ }^{141}$ Copyright 2015, Wiley. (o) High-magnification SEM images of the MoP-CA2 microstructure, and (p) polarization curves (in $0.5 \mathrm{M} \mathrm{H}_{2} \mathrm{SO}_{4}$ with a scan rate of $2 \mathrm{mV} \mathrm{s}^{-1}$ ) of MoP-CA2, MoP-CAO, Pt/C, and bare GCE. Reproduced with permission. ${ }^{64}$ Copyright 2014, Wiley.

offers a much higher surface roughness than Ni foil, which tends to promote more surface activity. ${ }^{87,88}$ Other reported common conductive substrates include Ti plate ${ }^{27,44,89}$ and $\mathrm{Cu}$ foam..$^{90}$

Metal chalcogenides. Metal chalcogenides such as sulfides $^{70,72,85,91}$ and selenides ${ }^{44,70,89,90,92,93}$ have demonstrated highly efficient performance in catalyzing HER-OER. Frequently investigated metal chalcogenides for water splitting include $\mathrm{Ni}-{ }^{70,86,94} \mathrm{Co}-{ }^{95,96} \mathrm{Mo}-\left(\mathrm{MoS}_{2}{ }^{97,98}\right.$ and $\left.\mathrm{MoSe}_{2}{ }^{99,100}\right)$ and $\mathrm{W}^{-101}$ chalcogenides. In 2013, our group disclosed that the cobalt sulfide (Co-S) film is effective as a HER-OER electrocatalyst with a faradaic efficiency of almost $100 \%$ in $1.0 \mathrm{M} \mathrm{KOH}^{102}$ The Co-S film exhibits a promising activity in the production of $\mathrm{H}_{2}$ from seawater. However, it suffers from stability issues in acidic medium $\left(0.5 \mathrm{M} \mathrm{H}_{2} \mathrm{SO}_{4}\right)$. Moreover, Co-S anchored on a conductive template such as Ti mesh has also revealed high catalytic activity and good stability as an OER electrocatalyst. ${ }^{70}$ Other adoptable conductive substrates include carbon-based materials such as carbon nanotubes (CNTs) and reduced graphene oxide (RGO). ${ }^{103,104}$ The interconnected CNT architecture offers improvement in porous characteristics while ensuring high electronic and mass transport. On the other hand, the RGO sheets are known to promote interfacial contact, thereby enhancing particle distribution and exposure of active sites.

Similar to the effect of bi-/multi-metallic elements on noble metal electrocatalysts, studies have shown that bi-/multimetallic sulfides have superior activity towards HER than their single component monometallic counterparts. ${ }^{96,101,105}$ However, the nature of the involved metal atoms and their respective compositions play a paramount role in the catalytic activities. For instance, $\mathrm{Ni}_{0.68} \mathrm{Co}_{0.32} \mathrm{~S}_{2}$ NWs displayed a lower HER activity than the undoped counterparts, $\mathrm{CoS}_{2}$ and $\mathrm{NiS}_{2} \cdot{ }^{96}$ On the other hand $\mathrm{Ni}_{0.33} \mathrm{Co}_{0.67} \mathrm{~S}_{2} \mathrm{NWs}$ grown on Ti foil synthesized via sulfurization of the $\mathrm{NiCo}_{2} \mathrm{O}_{4}$ precursor displayed the best HER activity in both neutral and alkaline media as compared to the undoped $\mathrm{CoS}_{2}$ and $\mathrm{NiS}_{2}{ }^{106}$ Similarly, our group demonstrated that the presence of $\mathrm{Co}\left(\mathrm{NiCo}_{2} \mathrm{~S}_{4} \mathrm{NA} / \mathrm{CC}\right)$ enhances the catalytic activity as witnessed from the observed current density of $100 \mathrm{~mA} \mathrm{~cm}{ }^{-2}$ at an overpotential of $305 \mathrm{mV}$ (for HER) and $340 \mathrm{mV}$ (for OER). The outstanding performance of $\mathrm{NiCo}_{2} \mathrm{~S}_{4} \mathrm{NA} / \mathrm{CC}$ was attributed to the high capacitance of the catalyst indicating a high surface roughness and surface area.

In an acidic medium, cobalt selenides are more stable electrocatalysts for water splitting than cobalt sulfides, especially when supported on carbon black,${ }^{96}$ carbon fiber paper (CFP) ${ }^{107}$ and carbon cloth (CC). ${ }^{108}$ Attributable features for the improved HER-OER activity include the increased surface area and boosted electrical conductivity. Other notable approaches for enhancing the catalytic efficacy of cobalt selenides include grafting or anchoring other components into the structure of the electrocatalysts. In this direction, numerous composites based on $\mathrm{CoSe}_{2}$ nanobelts with tremendous water splitting capacity have been developed. These include $\mathrm{Ni} / \mathrm{NiO} / \mathrm{CoSe}_{2}{ }^{109}$ and $\mathrm{MoS}_{2} / \mathrm{CoSe}_{2}{ }^{110}$ composites for the HER process, and $\mathrm{Mn}_{3} \mathrm{O}_{4} /$ $\mathrm{CoSe}_{2},{ }^{111} \mathrm{~N}$-doped graphene (NG)/CoSe${ }_{2}{ }^{112}$ and $\mathrm{CeO}_{2} / \mathrm{CoSe}_{2}{ }^{106}$ for the OER process. The enhanced OER and HER activities of these composites are ascribed to the synergetic chemical coupling effects.

Anchoring of the catalyst system on other components has been widely adopted to boost the catalytic performance of water splitting electrocatalysts. For example, Jin and coworkers introduced a high pressure and temperature hydrothermal continuous flow reactor (HCFR) for synthesizing NiCo LDHs on carbon fiber. The HCFR enabled the stable control of the reactor pressure that assisted the better tuning of the LDH size and morphology (Fig. 3(c)), which led to a current density of $10 \mathrm{~mA} \mathrm{~cm}{ }^{-2}$ at $367 \mathrm{mV}$ (vs. RHE) (Fig. 3(d)). ${ }^{113}$ On the other 
hand, Liu et al. showed that the $\mathrm{NiCo}_{2} \mathrm{~S}_{4}$ nanowire array on carbon cloth $\left(\mathrm{NiCo}_{2} \mathrm{~S}_{4} \mathrm{NA} / \mathrm{CC}\right)$ showed a better HER-OER performance than $\mathrm{NiCo}_{2} \mathrm{O}_{4} \mathrm{NA} / \mathrm{CC}$. This was because at the beginning of the OER process, $\mathrm{NiOOH}$ and $\mathrm{Co}(\mathrm{OH})_{2}$ formed at the surface of $\mathrm{NiCo}_{2} \mathrm{~S}_{4}$ which served as the active phases for the OER. $^{114}$

Furthermore, Feng and co-workers revealed that $\mathrm{Ni}_{3} \mathrm{~S}_{2}$ nanoarrays deposited on a good conductive substrate like nickel foam (NF) are active and stable bifunctional water splitting electrocatalysts (Fig. 3(e) and (f)). ${ }^{87}$ Similarly, a NiSe nanowire film anchored on nickel foam (NiSe/NF) fabricated by means of a hydrothermal reaction of $\mathrm{NF}$ and NaHSe was reported to be an efficient bifunctional water splitting electrocatalyst. However, theoretical evidence reveals that the harmonious effect of the interconnected architecture of catalyst's nanoarrays and the high-index planes is responsible for its unique electrocatalytic efficacy. ${ }^{70}$

For enriching the active sites, an interfaced $\mathrm{MoS}_{2} / \mathrm{Ni}_{3} \mathrm{~S}_{2}$ heterostructure engineered on $\mathrm{NF}$ as an advanced bifunctional electrocatalyst was developed. ${ }^{115}$ The generated abundant interfaces delivered $10 \mathrm{~mA} \mathrm{~cm}{ }^{-2}$ current density at a very low cell voltage of $1.56 \mathrm{~V}$ (vs. RHE). Coupled with theoretical calculations, it was revealed that the generated interfaces synergistically facilitated the chemisorption of $\mathrm{H}$ - and O-bound intermediates, thus expediting the overall water splitting process. Moreover, it was also confirmed that the induced lattice defects arising from the existence of $\mathrm{Co}_{9} \mathrm{~S}_{8}$ and $\mathrm{Ni}_{3} \mathrm{~S}_{2}$ combined phases highly contributed to the improved chemisorption of $\mathrm{H}$ and O-containing intermediates. ${ }^{116}$ On these defective heterointerfaces, DFT computation revealed a lower free energy for hydrogen $\left(\Delta G_{\mathrm{H}}\right)$ and hydroxide $\left(\Delta G_{\mathrm{OH}}\right)$, indicating a conducive active site for both HER and OER processes.

Metal phosphates and phosphides. In the past few decades, a myriad of transition metal based electrocatalysts have been used as potential water splitting electrocatalysts. ${ }^{70,73-75,117}$ Among these electrocatalysts, phosphates are one of the most studied bifunctional HER-OER electrocatalysts. ${ }^{24,37,118-120}$ In 2008, Nocera and co-workers reported an in situ synthesis approach for $\mathrm{Co}^{2+} /$ phosphate as OER catalysts by means of an indium tin oxide (ITO) substrate in $\mathrm{Co}^{2+}$-containing phosphate buffered saline (PBS). ${ }^{121,122}$ Nonetheless, the catalytic activity of this catalyst is pH-dependent. This is quite similar to the study of Artero and co-workers where a robust cobalt-phosphate-based electrocatalytic material (Janus $\mathrm{H}_{2}$-CoCat $\mathrm{NP}$ ) was developed for HER in a neutral electrolyte. ${ }^{123}$ Although with a remarkable catalytic activity, these NPs have to operate under near-neutral conditions.

Cobalt phosphides are suggested to be suitable catalysts for HER-OER. ${ }^{124-128}$ In 2014, Popczun and co-workers confirmed that CoP fabricated by means of a two-step colloidal synthesis approach is highly efficient and stable for HER in an acidic medium (Fig. 3(g)). Acting as a cathode in $0.5 \mathrm{M} \mathrm{H}_{2} \mathrm{SO}_{4}$, CoP NPs (electrodeposited on Ti foil) yielded a current density of $-20 \mathrm{~mA} \mathrm{~cm}^{-2}$ at $85 \mathrm{mV}$ (vs. RHE) overpotential (Fig. 3(h)) with a stability of $24 \mathrm{~h}$ (400 cyclic voltammetric sweeps). ${ }^{129}$ Another form of cobalt phosphide that can serve as a HER electrocatalyst is $\mathrm{Co}_{2} \mathrm{P}$ (Fig. 3(i) and (j)). ${ }^{130}$ However, this material was revealed to have a larger HER overpotential than CoP. The high Co/P ratio and low Co-P character make it unlikely for $\mathrm{Co}_{2} \mathrm{P}$ NPs to enable surficial distribution of conceivable active centers on the catalyst. ${ }^{131}$

Furthermore, following the catalytic efficacy of water splitting electrocatalysts due to the presence of phosphorus, various studies have demonstrated that incorporating metal catalysts with phosphorus can generate highly efficient HER-OER activity. ${ }^{71,85,132-134}$ For instance, Wei and co-workers revealed that the catalytic activity of $\mathrm{Ni}$ can be tuned with the extent of $\mathrm{P}$ deposition with the best activity observed at $10.8 \mathrm{wt} \% \mathrm{P}^{135}$ Moreover, pioneering studies conducted by our group showed that nickel phosphide (Ni-P) also demonstrated high activity for OER. Here, the authors established that a Ni-P nanoparticle film electrodeposited on $\mathrm{Ni}$ foam (Ni-P/NF) achieved a current density of $10 \mathrm{~mA} \mathrm{~cm}^{-2}$ at $1.67 \mathrm{~V}$ (vs. RHE) with $80 \mathrm{mV}$ and 309 $\mathrm{mV}$ overpotentials for HER and OER, respectively, in $1.0 \mathrm{M} \mathrm{KOH}$ (Fig. 3(k) and (l)). ${ }^{85}$ Other forms of nickel phosphides reported with high catalytic activity include $\mathrm{Ni}_{2} \mathrm{P},{ }^{136,137} \mathrm{Ni}_{5} \mathrm{P}_{4}{ }^{138}$ and $\mathrm{Ni} / \mathrm{Ni}_{8} \mathrm{P}_{3}{ }^{139}$ Most importantly, the $\mathrm{Ni}_{2} \mathrm{P}$ nanoarray has been demonstrated to be not only a high-performance non-noble-metal 3D catalyst electrode for hydrazine oxidation reaction ( $\mathrm{HzOR})$, but also a bifunctional catalyst material toward more energy-efficient hydrazine-assisted electrolytic hydrogen production. ${ }^{137}$

Aside from the common colloidal synthesis method for fabricating transition metal phosphides (TMPs), a recent surfactant-free low-temperature phosphidation approach by means of topotactic conversion of the corresponding precursors is developed. ${ }^{140}$ In this route, the propagation of CoP nanostructures is achieved through the use of conductive substrates to fabricate binder-free HER cathodes. A typical example is the synthesis of porous $\mathrm{CoP} / \mathrm{CC}$ with a rough surface using low-temperature phosphidation of the smooth-surfaced $\mathrm{Co}(\mathrm{OH}) \mathrm{F} / \mathrm{CC}$ precursor. The modified roughness of the surface was attributed to the dehydration and release of gases during the annealing of the precursor. The as-synthesized CoP/CC displayed a remarkable HER performance with an overpotential of 209, 106, and $67 \mathrm{mV}$ (vs. RHE) to afford a current density of $10 \mathrm{~mA} \mathrm{~cm}^{-2}$ in alkaline, neutral and acidic media, respectively. The remarkable performance can be partly related to the expedited electron transfer resulting from the improved interfacial contact between CoP and the CC conductive support, alongside the improved exposure of active centers. Similarly, our group reported the fabrication of $\mathrm{CoP}$ nanostructures decorated on carbon cloth (CoP/CC) via low-temperature phosphidation of the $\mathrm{Co}(\mathrm{OH}) \mathrm{F} / \mathrm{CC}$ precursor. ${ }^{57}$ When utilized as an electrocatalyst for HER, CoP/CC also displayed a remarkable performance in alkaline and neutral media. Encouraged by the performance of Co and Ni phosphides as bifunctional electrocatalysts and the merits of $3 \mathrm{D}$ structured catalysts, our group synthesized Ti-supported FeP nanowire arrays (FeP NA/Ti) to catalyze overall water splitting. ${ }^{77}$ The FeP NA served as the active site while $\mathrm{Ti}$ acted as the current collector. In an acidic medium, the FeP NA/Ti electrode displayed a low onset overpotential of $16 \mathrm{mV}$ with a Tafel slope of $38 \mathrm{mV} \mathrm{dec}{ }^{-1}$ and an 
exchange current density of $0.42 \mathrm{~mA} \mathrm{~cm}{ }^{-2}$. The good acidic stability of FeP NWs is depicted by the negligible changes in the overpotential after 2000 and 3000 cyclictest runs or $15 \mathrm{~h}$ of continuous operation. These attributes were related to the structure-induced electronic effect after phosphidation. After phosphidation, the 1D array format of FeP NWs is retained but the lengths and diameters are decreased (up to $600 \mathrm{~nm}$ ) and increased (50-95 $\mathrm{nm}$ ), respectively. Similarly, for exploiting the benefits of 3D architectured electrocatalysts, Wang and coworkers developed flexible 3D iron phosphide nanotubes (IPNTs) as a HER electrocatalyst. ${ }^{141}$ The prepared compound comprised of $\mathrm{FeP}$ coated with $\mathrm{NiFeO}_{x} / \mathrm{CFP}$ species yielded low onset overpotentials of 31 and $35 \mathrm{mV}$ ( $v s$. RHE) in alkaline and acidic solutions, respectively. Strangely, the in situ fabricated surficial iron oxide/phosphate species assisted the activation of OER with an onset overpotential of $250 \mathrm{mV}$ ( $v s$. RHE). Moreover, when the flexible 3D iron phosphide electrocatalyst was employed in a constructed alkaline electrolyzer, it displayed a good activity with a current density of $10 \mathrm{~mA} \mathrm{~cm}^{-2}$ at only $1.69 \mathrm{~V}$ (Fig. 3(m) and (n)). ${ }^{141}$ Another synthesis approach is the facile reaction of metal and bimetallic foils with several organophosphine sources to produce TMPs, proposed by Read and coworkers. ${ }^{142}$ The as-synthesized phosphides demonstrated outstanding OER and HER activity which compared favorably with samples prepared by means of more costly and elaborate procedures. Another TMP-based material considered as a water splitting electrocatalyst is MoP. Our group developed a closely interconnected network of MoP NPs with high specific surface area (SSA) as illustrated in Fig. 3(o). The synthesis of this microstructure was by a temperature-programmed reduction of the air-calcined precursor obtained from $\left(\mathrm{NH}_{4}\right)_{6} \mathrm{Mo}_{7} \mathrm{O}_{24}$. $4 \mathrm{H}_{2} \mathrm{O},\left(\mathrm{NH}_{4}\right)_{2} \mathrm{HPO}_{4}$ and citric acid (CA) with a Mo: $\mathrm{P}:$ CA molar ratio of $1: 1: 2$ (MoP-CA2). The MoP-CA2 NPs displayed a current density of $0.086 \mathrm{~mA} \mathrm{~cm}^{-2}$ with an onset overpotential of $40 \mathrm{mV}$, a Tafel slope of $54 \mathrm{mV} \mathrm{dec}^{-1}$, and an almost $100 \% \mathrm{FE}$ (Fig. 3(p)). In addition, a remarkable stability for 4000 cycles (at least $24 \mathrm{~h}$ ) was maintained. ${ }^{64}$ A most compelling feature is that metal phosphides are prone to oxidation and hence their surface turns into an oxide during OER catalysis. ${ }^{126}$ This newly formed oxide surface is the one that plays the true role of a catalyst. A recent study by Shifa et al. ${ }^{143}$ revealed that $\mathrm{Sn}$ doped $\mathrm{Ni}_{5} \mathrm{P}_{4}$ in situ transformed into more active $\mathrm{Sn}_{x} \mathrm{NiO}$ during the OER process. The electrochemically induced oxide is catalytically more active than the pristine oxides as it is formed in the vicinity of conductive phosphide species.

Metal nitrides. Metal nitrides have demonstrated superior HER-OER activities due to their good corrosion resistance and electrical conductivity. ${ }^{144}$ Comprehensive studies of this set of electrocatalysts are mostly focused on their intrinsic HER characteristics. ${ }^{145}$ Only a few studies have established the potential of metal nitrides to activate the OER. ${ }^{146}$ One among such studies is the synthesis of $\mathrm{Ni}_{3} \mathrm{~N}$ nanosheets, which were reported for the first time to be a suitable OER electrocatalyst when compared to NiO nanosheets. The improved intrinsic OER activity was attributed to the enhanced electrical conductivity with metallic characteristics and atomically disordered structure. ${ }^{147}$ On this account, it was suggested that metal nitride nanosheets could serve as bifunctional HER-OER electrocatalysts, where the chemical stability of nitrides deserves special attention in addition to their efficiency.

Despite the recent developments in improving the intrinsic OER behaviour, related studies in this regard, especially for overall water splitting, are limited. This may be related to the high overpotentials resulting from the restricted charge/ion transport. To circumvent this, a rational design to enhance the morphological effect, surface electrochemical reaction and electronic conductivity was proposed by Zhang and co-workers. Here, the authors synthesized Myriophyllum-like TiN@ $\mathrm{Ni}_{3} \mathrm{~N}$ nanowire arrays via a chemical bath deposition approach followed by an annealing process as a bifunctional HER-OER electrocatalyst. The as-synthesized TiN@ $\mathrm{Ni}_{3} \mathrm{~N}$ nanowire arrays displayed good HER and OER activities, and achieved a water splitting onset of $\sim 1.57 \mathrm{~V}$ with a current retention of $63.8 \%$ after $16 \mathrm{~h}$ of operation. ${ }^{148}$

Compared with single-metal nitrides, specific double metal nitrides have demonstrated better electrocatalytic activity and can be easily optimized by modulating the valence and electronic states of the metal elements. ${ }^{149,150}$ Cao and co-workers demonstrated this concept by revealing the enhanced HER activity and stability of cobalt molybdenum nitride $\left(\mathrm{Co}_{0.6} \mathrm{Mo}_{1.4} \mathrm{~N}_{2}\right)$ with a nanoscale morphology. Synthesized via a two-step solid-state reaction, $\mathrm{Co}_{0.6} \mathrm{Mo}_{1.4} \mathrm{~N}_{2}$ possessed a stacked four-layered sequence of mixed close-packed structures with alternating layers of transition metals in octahedral and trigonal prismatic coordination. Owing to this morphology, $\mathrm{Co}_{0.6} \mathrm{Mo}_{1.4} \mathrm{~N}_{2}$ with a low catalyst loading of $0.24 \mathrm{mg} \mathrm{\textrm {cm } ^ { - 2 }}$ achieved a current density of $10 \mathrm{~mA} \mathrm{~cm}{ }^{-2}$ at $-0.20 \mathrm{~V}$ (vs. RHE) under acidic conditions. ${ }^{149}$

Similarly, Jia and co-workers synthesized $\mathrm{Ni}_{3} \mathrm{FeN}$ nanoparticles $\left(\mathrm{Ni}_{3} \mathrm{FeN}-\mathrm{NPs}\right)$ by means of thermal ammonolysis of ultrathin NiFe-LDH nanosheets. The as-prepared NPs were highly effective for full water splitting owing to the unique electronic structure of the metallic composite, thereby facilitating charge distribution and $\mathrm{H}_{2} \mathrm{O}$ adsorption. ${ }^{151}$ Moreover, the particle size $(100 \mathrm{~nm})$ effect was alleged to boost the accessibility of active sites for the water splitting process.

Metal carbides. Similar to metal nitrides, metal carbides (also known as transition metal carbides (TMCs)) have a unique electronic structure, with which catalytic water splitting reactions can be accelerated through. Both theoretical and experimental verifications of improved electronic characteristics as a result of the hybridization of the d-orbitals of the metal and the sp-orbitals of carbon are provided. ${ }^{144,145}$ On this account, the d-band structure is broadened as this favors the hydrogen binding energy, thereby promoting the HER. ${ }^{145}$

Among this class of metals, molybdenum (Mo) and tungsten (W) carbides are so far the most investigated metal carbides for water splitting, ${ }^{152}$ with WC being the most stable in acidic solutions. ${ }^{153}$ On the other hand, $\mathrm{WC}, \mathrm{W}_{2} \mathrm{C}$, and $\mathrm{Mo}_{2} \mathrm{C}$ have similar passivation regions in alkaline/neutral medium. Overall, the stability of these compounds is influenced by the generation 
of surface oxide motifs in the considered $\mathrm{pH}$ range. ${ }^{152}$ Weigert and co-workers demonstrated the superior electrochemical stability of WC foil modified with a low coverage of Pt compared to that of pristine WC. ${ }^{153}$ The result showed that the stability of Pt-modified WC was sustained till a potential of $\sim 1.0 \mathrm{~V}$ (vs. NHE).

Among the different Mo carbides, ${ }^{145} \mathrm{Mo}_{2} \mathrm{C}$ is the most reported for water splitting and was previously explored as an effective noble-metal free electrocatalyst to replace Pt owing to its similar electronic characteristics to that of Pt and optimal hydrogen-adsorption properties. ${ }^{100,154,155}$ One major setback of this compound is its large particle size which acts as a limiting factor for the exposure of its active sites. In addition, the usage of these catalyst systems is challenged by surface oxidation or corrosion. ${ }^{153}$ So far, numerous approaches are proposed to promote particle miniaturization, and among those methods, dispersion is considered to be the most effective way to increase the surface area, enrich active sites and promote electron/mass activity. Recently, an enhanced electrocatalytic performance of $\mathrm{Mo}_{2} \mathrm{C}$ was demonstrated by embedding $\mathrm{Mo}_{2} \mathrm{C}$ nanoparticles in nitrogen-doped carbon nanosheet/graphene $\left(\mathrm{Mo}_{2} \mathrm{C} @ \mathrm{~N}-\mathrm{DC} / \mathrm{G}\right)$ aerogel films. ${ }^{156}$ The carbon nanomaterial has a high surface area which prevents the aggregation of $\mathrm{Mo}_{2} \mathrm{C}$ nanocrystals, as well as protects the metal catalysts from acid corrosion, enhances the stability in an acidic medium and simultaneously serves as "electron highways" for rapid electron transfer. ${ }^{157}$ Moreover, the heteroatom $\mathrm{N}$ dopant modifies the surface chemistry with different defects and alters the electronic structure of the catalysts, leading to optimized adsorption energy of the key intermediates on the surface. The synergistic effect of $\mathrm{N}$-doped carbon nanomaterials and $\mathrm{Mo}_{2} \mathrm{C}$ nanoparticles enriched the electron density on the carbon surface and promoted hydrogen adsorption as well as evolution.

In summary, the above discussed studies have evidently shown that the performances of metal-based electrocatalysts (as listed in Table 1) for water splitting are dependent on several key parameters. First, their overall HER-OER activity mostly depends on the structure and specific active surface area of the catalyst system rather than the nature of the constituting metal. This deduction elaborates the importance of morphology engineering and composition towards the optimization of the density and distribution of catalytic active sites. Second, the electrolyte selection, which is also critical to efficiency and stability, is dependent on its ion species, concentration, $\mathrm{pH}$ values and the suitability with electrocatalysts. Since the electrolyte would directly affect the reaction kinetics and an inappropriate electrolyte would cause corrosion of the electrode, choosing a suitable electrolyte has a great influence on the performance and stability of the electrocatalysts. Third, the lifetime of a catalyst directly affects its practical application. The economic strategies for catalyst reactivation so as to further extend the shelf life of a catalyst will also play an important role in developing commercializable technologies. Additionally, the cost from material elements, catalyst preparation, electrolytes, and electrodes will decide the upscalability and hence the practicality of this technology. Although non-noble metals are preferred to minimize element cost, noble metals would be still competitive if their cost-effectiveness surpasses non-noble candidates, i.e. a tiny amount of noble metals, for example in the monodispersed single-atom catalysts, could enhance catalysis and stability greatly.

2.2.3 Defected functional electrocatalysts. As an alternative to metal-based catalyst systems, defected functional materials with the potential to catalyze HER and OER processes, such as functional carbon materials such as carbon nanotubes/nanofibers ${ }^{158}$ and graphene-based nanosheets, ${ }^{159}$ have captivated researchers in this field. In addition to the favorable intrinsic catalytic characteristics, functional carbon catalysts display a more exceptional catalytic quality when doped with non-metal heteroatoms. ${ }^{160}$ Under this circumstance, the electronic structure of the carbon species is altered with an increase in surface defects, which often act as the catalytic active sites. ${ }^{161,162}$ The harmonious effect of the enriched active sites resulting from the doping and the intrinsic properties of the functional carbon materials portrays this catalyst system as a suitable candidate for an energy-saving electrochemical system.

However, the investigation of the bifunctional HER and OER activity on these carbon surfaces is still limited. Therefore, exploring the application of functional carbon-based materials for overall water splitting cannot be over-exaggerated. In this regard, Lai and coworkers first conducted an extensive study on the fabrication of porous graphite nanocarbons co-doped with $\mathrm{O}, \mathrm{N}$ and $\mathrm{P}$ heteroatoms as a self-supported 3D electrode (ONPPGC/OCC) for overall water splitting at various $\mathrm{pH}$ values. ${ }^{163}$ For instance, in an alkaline medium, ONPPGC/OCC electrocatalysts displayed good HER and OER activities. The electrolyzer attained a current density of $10 \mathrm{~mA} \mathrm{~cm} \mathrm{~cm}^{-2}$ at a cell voltage of $1.66 \mathrm{~V}$ with remarkable stability. The remarkable electrocatalytic performance of the porous nanocarbon was attributed to the unique 3D structure and pore distribution, highly dispersed active sites, improved transport properties, and good electrical conductivity. Qiao and co-workers presented a 3D-architectured hydrated catalyst NiCo LDH on N-doped graphene hydrogels (NG-NiCo) synthesized through ammonia-involved hydrothermal treatment of a graphene hydrogel followed by heterogeneous deposition of the obtained NiCo hydroxide on NG. ${ }^{164}$ While the presence of N-dopant reduces the catalyst's internal resistance and the graphene provides a porous $3 \mathrm{D}$ interconnected network, the synergistic metal-O-C and metal-N-C interactions yield interfacial active centers to activate the HER-OER. Undeniably, this study offers a unique exciting means to explore functional carbon compounds as water splitting electrocatalysts. The electrocatalytic performance of ONPPGC/OCC in other media is presented in Table 1.

Another widely used carbon-based material is graphdiyne (GDY), a novel plane carbon network consisting of $\mathrm{sp}-/ \mathrm{sp}^{2}-\mathrm{co}$ hybridized carbon atoms. ${ }^{182}$ This porous carbon network with a unique intrinsic band gap, excellent electric conductivity, and strong stability was first synthesized by $\mathrm{Li}$ and co-workers in $2010 .{ }^{183}$ Given its intrinsic properties, the authors prepared a GDY anchored on $\mathrm{CoN}_{x}$ nanosheets with a seamless interacting 
interface on Ni foam $\left(\mathrm{CoN}_{x} @ G D Y \mathrm{NS} / \mathrm{NF}\right) .{ }^{182}$ When tested in 1.0 M KOH, CoN ${ }_{x} @ G D Y$ NS/NF attained a current density of $10 \mathrm{~mA} \mathrm{~cm}{ }^{-2}$ with a cell voltage of $1.48 \mathrm{~V}$ when employed as a bifunctional electrocatalyst. Similarly, Si and co-workers utilized GDY to develop a hierarchical heterostructure composite with NiFe LDH anchored on copper foam (GDY@NiFe-LDH/CF) to catalyze the overall water splitting. ${ }^{184}$ In its function, GDY@ $\mathrm{NiFe}-\mathrm{LDH} / \mathrm{CF}$ attained a current density of $20 \mathrm{~mA} \mathrm{~cm}{ }^{-2}$ with a cell voltage of $1.512 \mathrm{~V}$. The remarkable electrochemical performance was credited to the improved interfacial chemical interaction between $\mathrm{Fe}, \mathrm{Ni}$ and the triple $\mathrm{C}-\mathrm{C}$ bonds in GDY. This interaction facilitated an improved electron charge distribution with a controlled diffusion rate. ${ }^{184}$ In addition, Table 1 presents a summary of other adopted metal-free electrocatalysts.

Benefitting from the synergistic effect of metallic behaviour, interconnected pores of nanowire arrays, and a distinct 3D electrode structure, $\mathrm{Co}_{4} \mathrm{~N}$ porous nanowire arrays on carbon cloth attained a low overpotential of $\sim 0.26 \mathrm{~V}$ at $10 \mathrm{~mA} \mathrm{~cm} \mathrm{~cm}^{-2}$ and a Tafel slope of $44 \mathrm{mV} \mathrm{dec}{ }^{-1}$ in an alkaline medium. Moreover, theoretical evidence shows that the metallic $\mathrm{Co}_{4} \mathrm{~N}$ core with a thin cobalt oxides/hydroxides shell serves as the active centre during the OER process. ${ }^{146}$

In short, defected functional catalysts would still need the metal-based materials for the synergistic catalysis and/or as the supporting template. Besides laterally referenceable perspectives discussed above for metal-based catalysts, the synergy of interface coupling between metal-free and metallic components will play a significant role in enabling an efficient carrier (electrons in HER and holes in OER) transportation within the catalysts, thus affecting the performances.

\section{Ammonia synthesis via $\mathrm{N}_{2}$ reduction reaction}

Ammonia $\left(\mathrm{NH}_{3}\right)$ is a commonly synthesized chemical across the world. It can function as a building block for the production of other N-based compounds and as a clean energy carrier due to its high H-content. ${ }^{185,186}$ Presently, $\mathrm{NH}_{3}$ is industrially fabricated via the Haber-Bosch process involving an exothermic interaction between $\mathrm{N}_{2}$ and $\mathrm{H}_{2}\left(\mathrm{~N}_{2}+3 \mathrm{H}_{2} \rightarrow 2 \mathrm{NH}_{3}\right)$ in the presence of a catalyst at high pressure and high temperature. ${ }^{187}$ Theoretically, compared with Fe-based catalysts, Ru-based catalysts are more effective to operate the Haber-Bosch process under a relatively mild condition (pressure $\leq 100$ bar). ${ }^{188}$ Despite this, the thermodynamics is still low (approximately 10-15\%) with an energy implication estimated at $1-2 \%$ of global annual energy ${ }^{186,189}$ and the involvement of $\mathrm{H}_{2}$ in this process is undermined by the consumption of fossil fuels (with sizeable $\mathrm{CO}_{2}$ emissions). ${ }^{190}$

Recently, electrocatalytic $\mathrm{N}_{2}$ reduction reaction (NRR) is viewed as an energy-saving approach for $\mathrm{NH}_{3}$ production, as the process of synthesizing $\mathrm{NH}_{3}$ is carried out under ambient temperature and pressure. Hence, electrocatalytic NRR is considered as an eco-friendly and energy-conservation approach for $\mathrm{NH}_{3}$ synthesis. However, its practical application is still constrained by the costly electrolytes, low $\mathrm{NH}_{3}$ yields and so on. ${ }^{188}$ On this account, a knowledge-driven guide towards the development of efficient electrocatalysts is a fundamental step for realizing electrocatalytic $\mathrm{N}_{2}$ fixation and accelerated NRR processes. Characteristically, NRR electrocatalysts are of three types: biocatalysts, homogeneous, and heterogeneous. The biocatalysts and homogeneous electrocatalysts contain ligandsurrounded metal centers, ${ }^{191}$ which poses a limitation due to the high cost of the ligands. Synthesis challenges as well as the low electrical properties hinder the development of these types of catalysts. ${ }^{192}$ On the other hand, heterogeneous electrocatalysts are highly durable and are more integrable with functional energy conversion devices. ${ }^{193}$ On this account, the design and development of heterogeneous electrocatalysts have been exploited for $\mathrm{NH}_{3}$ synthesis.

In this review, recent experimental and theoretical insights into the development of NRR electrocatalysts are highlighted. Particular emphasis will be devoted to the significance and implications of recent developments. First, the electrochemical NRR mechanisms are discussed, providing idealistic modalities for enhancing catalytic activity, selectivity and stability. Based on these mechanisms, various heterogeneous electrocatalysts are reviewed in terms of catalytic performance, reflecting different accumulated outcomes and mechanistic understanding of catalyst design principles. Here, electrocatalysts including metal (noble and non-noble) catalysts and metal-free catalysts are discussed.

\subsection{Electrochemistry of $\mathbf{N}_{2}$ reduction reaction (NRR)}

3.1.1. Fundamentals of NRR. Generally, the electroreduction of nitrogen on a heterogeneous catalyst involves two major reaction mechanisms: dissociative and associative (Fig. 4). The dissociative mechanism involves breaking of the $\mathrm{N} \equiv \mathrm{N}$ bond of the $\mathrm{N}_{2}$ molecule to form $\mathrm{N}$-adatoms on the surface of the catalyst prior to the protonation process. Subsequent protonation of the adatoms on the surface results in the yield of $\mathrm{NH}_{3}$ independently. Fig. 4(a) presents a detailed schematic representation of the mechanistic pathway, with a characteristic $\Delta G$ plot to illustrate the minimum energy pathway (MEP) on the Ru NP catalyst (Fig. 4(b)). ${ }^{194}$ Deductions from the mechanistic pathway and the characteristic $\Delta G$ plot suggest that the last reduction step $\left({ }^{*} \mathrm{NH}_{2}+\mathrm{H}^{+}+\mathrm{e}^{-} \rightarrow{ }^{*} \mathrm{NH}_{3}, \Delta \Delta G=0.32 \mathrm{eV}\right.$ uphill) is the rate-determining step. ${ }^{195}$ On the other hand, in the associative mechanism, the two $\mathrm{N}$ centers in $\mathrm{N}_{2}$ remain intact as a molecule while being protonated, with the release of $\mathrm{NH}_{3}$ after the severance of the $\mathrm{N}-\mathrm{N}$ bond (Fig. 4(c)). This mechanism is further characterized based on the order of protonation of the two $\mathrm{N}$ centers (with the assumption that the $\mathrm{N}_{2}$ molecule is adsorbed on the catalyst). The protonation sequence and its associated energies will dictate the rate-determining reaction on the catalyst surface. Fig. 4(d) illustrates that the first reduction process $\left({ }^{*} \mathrm{~N}_{2}+\mathrm{H}^{+}+\mathrm{e}^{-} \rightarrow{ }^{*} \mathrm{~N}_{2} \mathrm{H}, \Delta G=1.03 \mathrm{eV}\right.$ uphill) is the rate-determining step of the associative reaction on the Ru NP catalyst. ${ }^{194}$

Generally, protonation of the $\mathrm{N}$ centers occurs via two routes in the associative reaction. In the first instance, the $\mathrm{N}$ center 

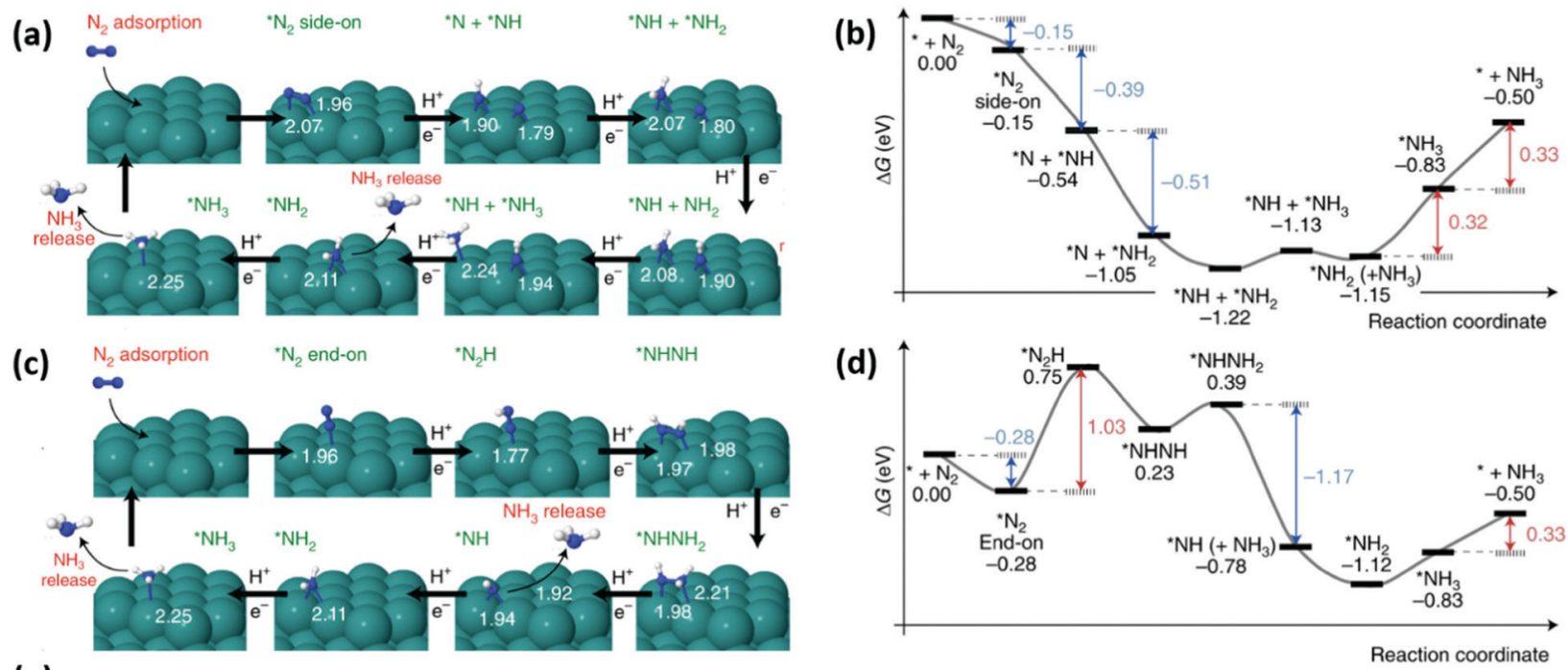

(e)

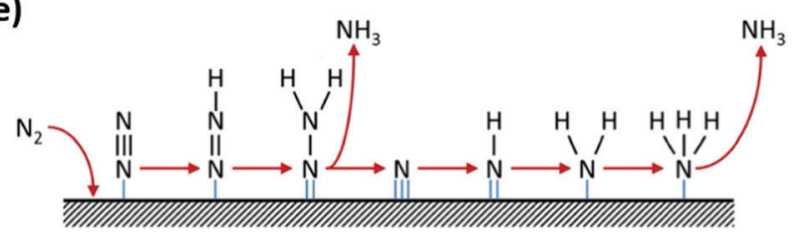

(f)

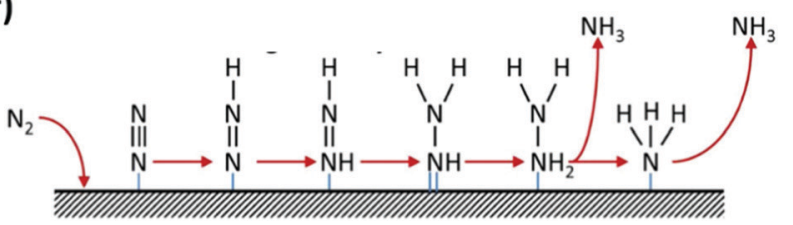

Fig. 4 NRR mechanisms on heterogeneous catalysts. (a) Dissociative mechanism and its (b) characteristic Gibbs free energy plot illustrating the minimum energy pathway on Ru nanoparticles (Ru-NPs). (c) Associative mechanism and its (d) characteristic Gibbs free energy plot illustrating the minimum energy pathway on Ru nanoparticles (Ru-NPs). Images (a)-(d) are reproduced with permission. ${ }^{194}$ Copyright 2019, Nat. Catal. Comparison of (e) distal and (f) alternative associative mechanisms. Images (e) and (f) are reproduced with permission. ${ }^{196}$ Copyright 2017, Elsevier.

farther from the catalyst surface is preferentially protonated (assuming an end-on coordination mode for the $\mathrm{N}_{2}$ molecule) resulting in the yield of $\mathrm{NH}_{3}$ and formation of a metal nitride (M-N) motif, which is later protonated to yield the second equivalent $\mathrm{NH}_{3}$. This is classified as the distal associative pathway (Fig. 4(e)). On the other hand, the alternative route relates to the protonation of each $\mathrm{N}$ center alternately until an $\mathrm{N}$ center is completely hydrogenated to $\mathrm{NH}_{3}$ and the $\mathrm{N}-\mathrm{N}$ bond is severed (Fig. 4(f)). ${ }^{196}$

In both dissociative and associative mechanisms, electrochemical NRR involves a series of proton-electron transfer steps with the formation of multiple intermediates: ${ }^{197}$

$$
\begin{array}{r}
\mathrm{N}_{2}+\mathrm{H}^{+}+\mathrm{e}^{-} \leftrightarrow \mathrm{N}_{2} \mathrm{H} \quad E^{0}=-3.2 \mathrm{~V} v s . \mathrm{RHE} \\
\mathrm{N}_{2}+2 \mathrm{H}^{+}+2 \mathrm{e}^{-} \leftrightarrow \mathrm{N}_{2} \mathrm{H}_{2}(\mathrm{~g}) \quad E^{0}=-1.10 \mathrm{~V} v s . \mathrm{RHE} \\
\mathrm{N}_{2}+4 \mathrm{H}^{+}+4 \mathrm{e}^{-} \leftrightarrow \mathrm{N}_{2} \mathrm{H}_{4}(\mathrm{~g}) \quad E^{0}=-0.36 \mathrm{~V} \text { vs. RHE } \\
\mathrm{N}_{2}+4 \mathrm{H}_{2} \mathrm{O}+6 \mathrm{e}^{-} \leftrightarrow \mathrm{N}_{2} \mathrm{H}_{4}+4 \mathrm{OH}^{-} \quad E^{0}=+0.55 \mathrm{~V} v s . \mathrm{RHE} \text { at } \mathrm{pH} \\
14 \\
\mathrm{~N}_{2}+\mathrm{e}^{-} \leftrightarrow \mathrm{N}_{2}{ }^{-} \text {(aq) } \quad E^{0}=-3.37 \mathrm{~V} \text { vs. RHE at } \mathrm{pH} 14
\end{array}
$$

It can be deduced that in a neutral solution $(\mathrm{pH}=0)$, reactions (31) and (32) are the most negative than $\mathrm{H}^{+}$reduction $\left(E^{0}=0 \mathrm{~V}\right.$ vs. SHE at $\mathrm{pH}=0),{ }^{197}$ which implies that it is more difficult to form $\mathrm{N}_{2} \mathrm{H}$ and $\mathrm{N}_{2} \mathrm{H}_{2}$ intermediates. However, in a strong alkaline solution ( $\mathrm{pH}=14)$, it is likely that reactions (31) and (35) will occur simultaneously, thus, limiting the formation of the intermediate $\mathrm{N}_{2} \mathrm{H}$. Besides, the lower redox potentials of the
$4 \mathrm{e}^{-}$-reduction (reaction (33)) and $6 \mathrm{e}^{-}$-reduction (reaction (34)) paths when compared to the $1 \mathrm{e}^{-}$-reduction and $2 \mathrm{e}^{-}$-reduction paths result from the weak bond strengths of the second and third bonds of the $\mathrm{N}_{2}$ molecule. To date, a generally acceptable NRR mechanism is yet to be thoroughly elaborated upon due to the intricacies involving the formation of multiple intermediates and multi-step electron/proton transfer. Naturally, the NRR is achieved by means of nitrogenase enzymes in a contrary process to the Haber-Bosch process. One of the most reported nitrogenases are the FeMo nitrogenases ${ }^{198}$ with the NRR undergoing an associative mechanism (with the $\mathrm{N}_{2}$ molecule coordinating to a metal centre in the FeMo nitrogenase in the end-on mode). However, this is still highly debated to this day. ${ }^{199}$

Irrespective of the operating mechanism, it is evident that these enzymes are suitable catalysts for $\mathrm{NH}_{3}$ production under ambient conditions in aqueous media with an exciting energy efficiency. Consequently, electrochemistry has appeared as an attractive approach adopting the HER of the water splitting process to produce $\mathrm{H}^{+}$and $\mathrm{e}^{-}$for the reduction of nitrogen (from air). Renewable energy sources are also suitable to power the reaction operation. Similar to the water splitting process, the NRR mechanism also depends on the nature of the electrolyte. Depending on the nature of the electrolyte, the general reactions are as follows:

In an acidic condition:

At the cathode:

$$
\mathrm{N}_{2}+6 \mathrm{H}^{+}+6 \mathrm{e}^{-} \rightarrow 2 \mathrm{NH}_{3}
$$


At the anode:

$$
3 \mathrm{H}_{2} \mathrm{O} \rightarrow 3 / 2 \mathrm{O}_{2}+6 \mathrm{H}^{+}+6 \mathrm{e}^{-}
$$

In an alkaline condition:

At the cathode:

$$
\mathrm{N}_{2}+6 \mathrm{H}_{2} \mathrm{O}+6 \mathrm{e}^{-} \rightarrow 2 \mathrm{NH}_{3}+6 \mathrm{OH}^{-}
$$

At the anode:

$$
6 \mathrm{OH}^{-} \rightarrow 3 / 2 \mathrm{O}_{2}+3 \mathrm{H}_{2} \mathrm{O}+6 \mathrm{e}^{-}
$$

Overall reaction:

$$
\mathrm{N}_{2}+3 \mathrm{H}_{2} \mathrm{O} \rightarrow 3 / 2 \mathrm{O}_{2}+2 \mathrm{NH}_{3}
$$

Firstly, the carbon footprint in the generation of ammonia is minimized. In addition, exploiting the intrinsic characteristics and flexibility of an electrochemical system increases the potential of pilot/large-scale $\mathrm{NH}_{3}$ synthesis. Moreover, feasibility studies on a pilot scale are necessary for the successful commercialization of this process. From a techno-economic standpoint, the electrochemical NRR process saves more energy with an estimated $20 \%$ increased energy efficiency compared to the Haber-Bosch scheme, considering coal as the source of hydrogen. ${ }^{200}$ However, this potential might be challenged by the limited depth of material knowledge and scale-up dynamics of current catalyst-development strategies.

3.1.2. Recent theoretical insight into NRR. Recently, the electrosynthesis of ammonia has been extensively supported from a theoretical perspective. Most importantly, these studies focus on material discovery for suppressing the HER process in favor of $\mathrm{N}_{2}$ protonation. ${ }^{195}$ With this issue under consideration, it is important to review related theoretical studies towards future schemes to enhance electrochemical NRR and retardation of the HER process.

A milestone study utilizing computational SHE via harmonic approximation and DFT calculations to investigate the reduction energetics for $\mathrm{N}_{2}$ (ad-molecules and adatoms) on different transition metal surfaces in an acidic medium was conducted by Nørskov and co-workers in 2012. ${ }^{201}$ By correlating the chemisorption energies with the reaction intermediates under the assumption that the change in free energy is proportional to the activation energy barrier in each elementary step, a volcano plot was obtained. The segmentation of the volcano plot into right and left legs indicates that the metals on each leg possess a weak and a strong N-binding energy, respectively. For instance, the metals on the left-leg ( $\mathrm{Sc}, \mathrm{Y}, \mathrm{Ti}$ and $\mathrm{Zr}$ ) have strong affinity for $\mathrm{N}$-adatoms, which will facilitate significant synthesis of $\mathrm{NH}_{3}$ relative to $\mathrm{H}_{2}$, particularly at a bias of $-1 \mathrm{~V}$ to $-1.5 \mathrm{~V}$ (vs. SHE). At the top of the volcano plot are the most active metals for ammonia synthesis, which include Mo, Fe, Rh, and $\mathrm{Ru}$. However, the faradaic efficiency (FE) of these metals is low owing to the substantial HER competition. ${ }^{201}$ Also, the authors revealed that the defect-free surfaces of TMs are catalytically more active for $\mathrm{NH}_{3}$ formation than their stepped counterparts due to the lower onset potential on the close-packed flat surfaces compared to the stepped surfaces. ${ }^{201}$
Generally, the production of $\mathrm{NH}_{3}$ is simply catalyzed by pure transition metals (TMs). Still, the $\mathrm{N}$-adsorption energies $(\Delta E)$ of these metals are insufficient relative to the ideal values within the range of $-0.4 \mathrm{eV}$ to $-1.4 \mathrm{eV} .^{202}$ Contrarily, some early TMs (including Sc, Y, Ti and $\mathrm{Zr}$ ) have displayed strong N-bonding within the bias range of $-1 \mathrm{~V}$ to $-1.5 \mathrm{~V}$ (vs. SHE). Nonetheless, these early TMs change their phase from metallic state to bulk nitrides, which hinders the potential of $\mathrm{N}_{2}$ activation due to the widened d-band centers from the Fermi level. ${ }^{202}$ For example, utilizing iron catalysts in $\mathrm{NH}_{3}$ production gives rise to the formation of a $\mathrm{Fe}_{4} \mathrm{~N}$-like structure, although it is unsure if the $\mathrm{N}$-species is an ad-molecule or adatom. ${ }^{203}$

Pertaining to boosting the reduction of $\mathrm{N}_{2}$ while simultaneously suppressing the HER process, $\mathrm{Li}$ and co-workers proposed an electron-deficient approach to retard the HER process in an alkaline medium under ambient conditions. Here, the authors boosted the NRR performance of Cu NPS with negligible catalytic activity via a local electron depletion effect using Mott-Schottky rectifying contact with a polyimide support. The electron-depleted Cu NPs considerably enhanced $\mathrm{N}_{2}$ pre-adsorption leading to a better $\mathrm{NH}_{3}$ yield. This approach of inciting an electron deficient surface offers a novel insight into the rational design of inexpensive NRR catalyst systems with high activity and selectivity. ${ }^{204}$

Moreover, early TMs are readily oxidized to form their respective oxides in their natural state which may alter their catalytic efficacy. ${ }^{205}$ On this account, the performance of TM oxides was theoretically investigated towards electrosynthesis of $\mathrm{NH}_{3}$ under ambient conditions. Skúlason and co-workers studied the potential of TM dioxides as NRR electrocatalysts and revealed that the (110) planes of $\mathrm{ReO}_{2}, \mathrm{NbO}_{2}$, and $\mathrm{TaO}_{2}$ are the best suited for $\mathrm{NH}_{3}$ synthesis given their reasonably low onset potentials of $-0.57 \mathrm{~V},-1.07 \mathrm{~V}$ and $-1.21 \mathrm{~V}$ ( $v s$. SHE), respectively. ${ }^{206}$ The least overpotential was exhibited by $\mathrm{IrO}_{2}$ $(-0.36 \mathrm{~V})$ but this catalyst preferentially adsorbs H-adatoms which will favor the HER process. ${ }^{206}$

Lately, the possible production of $\mathrm{NH}_{3}$ using $\mathrm{NbO}_{2} \mathrm{NPs}$ as an electrocatalyst has been experimentally proven under ambient conditions. $^{207}$ This possibility is accredited to the electronic characteristics of $\mathrm{NbO}_{2}$ which are alleged to promote $\mathrm{NH}_{3}$ fixation. ${ }^{188}$ Owing to the nature of $\mathrm{Nb}^{4+}, \mathrm{NbO}_{2}$ NPs possess an empty d-orbital that readily accepts electrons leading to a strong surficial bonding with $\mathrm{N}_{2}$. In addition, the activation of $\mathrm{N}$-admolecules/adatoms could be promoted by the back donation of the single d-electron from the $\mathrm{Nb}^{4+}$ cation. In this respect, the $\mathrm{NbO}_{2} \mathrm{NPs}$ attained an $\mathrm{NH}_{3}$ yield rate of $11.6 \mu \mathrm{g} \mathrm{h}^{-1} \mathrm{mg}^{-1}$ cat. with an FE of $32 \%$ at -0.60 (vs. RHE) and -0.65 (vs. RHE), respectively, when tested in a $0.05 \mathrm{M}$ $\mathrm{H}_{2} \mathrm{SO}_{4}$ solution. ${ }^{208}$ Similarly, other forms of niobium oxides have also shown potential applications as possible NRR electrocatalysts. ${ }^{209,210}$ For example, our group demonstrated the effectiveness of $\mathrm{Nb}_{2} \mathrm{O}_{5}$ as an electrocatalyst for $\mathrm{NH}_{3}$ production as both nanofibers ${ }^{209}$ and nanowires. ${ }^{210}$

Nevertheless, metals have shown good potential for $\mathrm{N}_{2}$ reduction under ambient conditions; it is worthwhile to mention that some metals and their respective oxides do not display 
similar catalytic efficacy particularly at room temperature. This is somewhat related to their low conductivity ${ }^{192}$ and in other cases, it is due to their strong affinity for hydrogen. ${ }^{202}$ For the latter, typical examples are the late TMs which greatly enable the HER, thereby fettering the formation of $\mathrm{NH}_{x}$ intermediates. ${ }^{202}$ Unlike these metals, some metals especially the middle TMs offer moderate binding energies for N-motifs, thus making them suitable candidates for $\mathrm{N}_{2}$-to- $\mathrm{NH}_{3}$ conversion. ${ }^{211}$ Proceeding from this, it is likely that the best suited electrocatalyst can be designed by selecting the right materials with intrinsic NRR characteristics. Otherwise, it is necessary to adopt measures to promote the NRR performance of the catalyst system.

An effective scheme is to incorporate a second active center to facilitate the mechanistic spillover and hydrogenation of the activated N-motifs from the metal surface. For instance, Wang and co-workers proposed the use of $\mathrm{LiH}$ as a second active center to disrupt the NRR pathway on a TM or its nitride (TMN) by spilling over the activated $\mathrm{N}$-motifs to its surface for direct hydrogenation. $\mathrm{LiH}$ is a powerful reducing agent which offers immediate $\mathrm{H}$-species to bind with $\mathrm{N}$-motifs to yield $\mathrm{LiNH}_{2}$. Subsequently, $\mathrm{LiNH}_{2}$ heterolytically splits to release $\mathrm{H}_{2}$ and $\mathrm{NH}_{3}$ with the regeneration of $\mathrm{LiH}$. It should be emphasized that this rational scheme is an approach to improve the NRR performance of early and late $3 \mathrm{~d}$ TMs under ambient conditions. ${ }^{202}$ However, in realistic terms, the two active centers need to be appropriately separated.

Alternatively, HER-retarding strategies are equivalently being suggested for a successful headway in the NRR process. In this regard, a common approach is the use of electrocatalysts other than pure metals. The introduction of non-metal species on metals has shown remarkable results pertaining to $\mathrm{NH}_{3}$ synthesis. A recent theoretical study involving metal nitrides as NRR electrodes suggests that $\mathrm{VN}$ and $\mathrm{ZrN}$ produced $\mathrm{NH}_{3}$ at potentials of $-0.51 \mathrm{~V}$ ( $v s$. NHE) and $-0.76 \mathrm{~V}$ ( $v s$. NHE), respectively. These results were unachievable when the nitrides were veiled with H-adatoms. ${ }^{205}$ Similarly, Abghoui and co-workers reported a parallel result for $\mathrm{NbN}$ and $\mathrm{CrN} .^{212}$

To date, there are no extensive computational and experimental studies investigating the efficacy of metal nitrides as catalysts for $\mathrm{N}_{2}$-to- $\mathrm{NH}_{3}$ conversion. However, Simonov and co-workers critically assessed the electrocatalytic activity of $\mathrm{VN}$ and $\mathrm{Nb}_{4} \mathrm{~N}_{5}$ anchored on CC under various conditions. ${ }^{213}$ Here, the authors concluded that polycrystalline $\mathrm{VN}$ and $\mathrm{Nb}_{4} \mathrm{~N}_{5}$ are electrocatalytically inactive toward $\mathrm{NH}_{3}$ synthesis regardless of the operating condition. Another related study involved the computational investigation of single TMs anchored on boron nitride (TM-BN) as a $\mathrm{N}_{2}$ fixation electrocatalyst. ${ }^{193}$ Of the investigated single TMs ( $\mathrm{Sc}$ to $\mathrm{Zn}, \mathrm{Mo}, \mathrm{Ru}, \mathrm{Rh}, \mathrm{Pd}$, and $\mathrm{Ag}$ ), the highest electrocatalytic activity was witnessed on the defective Mo-BN nanosheet with a low overpotential of $0.19 \mathrm{~V}$. The high spin-polarization, selective $\mathrm{N}_{2} \mathrm{H}^{*}$-motif stabilization and $\mathrm{NH}_{2}{ }^{*}$-motif destabilization are attributed for the high catalytic activity of the Mo-BN nanosheet for $\mathrm{N}_{2}$-to- $\mathrm{NH}_{3}$ conversion. ${ }^{193}$

Equally, it will be beneficial if similar theoretical studies are conducted on binary nitrides. For instance, $\mathrm{Co}_{3} \mathrm{Mo}_{3} \mathrm{~N}$ is one the most active electrocatalysts for $\mathrm{NH}_{3}$ synthesis ${ }^{214}$ and was recently modeled for the conventional Haber-Bosch process. ${ }^{215}$ On a broader perspective, extensive theoretical studies are required to understand the NRR mechanism over the numerously designed electrocatalysts. Despite the countless NRR electrocatalysts proposed from laboratory tests, their current status is still unclear due to the lack of in-depth theoretical studies. These studies, although limited, are mandatory for a thorough comprehension of the reaction mechanism. Even though DFT computations may suffice as a good theoretical approximation of the catalytic activity, kinetics and evaluation of the optimization scheme, the findings may not be precise experimentally. Therefore, the need for a thorough assessment of NRR electrocatalysts, supported by both experimental and theoretical studies, is crucial.

\subsection{Electrocatalysts for NRR}

An important milestone in $\mathrm{NH}_{3}$ electrosynthesis was the establishment of $\mathrm{NH}_{3}$ production in aqueous solution under ambient conditions from $\mathrm{N}_{2}$ and $\mathrm{H}_{2}$ via a back-to-back cell configuration by Furuya and Yoshiba in $1990 .{ }^{14}$ Of the 26 cathodes studied, ZnSe was the most suitable for the reduction of $\mathrm{N}_{2}$ with an $\mathrm{NH}_{3}$ electrode area-normalized yield rate of $0.23 \mathrm{~mol} \mathrm{~h}^{-1} \mathrm{~m}^{-2}$, attaining an $\mathrm{FE}$ of $1.3 \%$ at a potential of $-1 \mathrm{~V}$ ( $v s$. RHE). ${ }^{14}$ Despite the high yield rate, the FE is relatively low and was attributed to the predominant cathodic reduction of $\mathrm{H}_{2} \mathrm{O}$ at the high negative cell potential, particularly in an aqueous solution where the concentration of $\mathrm{H}_{2} \mathrm{O}$ is relatively high. For this reason, the suppression of the HER is considered by most to be the utmost challenge confronting electrochemical NRR.

Moreover, the determination of $\mathrm{N}_{2}$ production in NRR is more challenging than $\mathrm{H}_{2}$ or $\mathrm{O}_{2}$ production in water splitting due to the possible contamination from the ambient environment and low production rates. ${ }^{216,217}$ Concretely, the ammonia detected may come from other routes beyond the NRR, such as ammonia contamination in the feeding gas, electrolyte and electrode surface, and decomposition or desorption from the catalyst itself, especially in the case of N-containing materials. ${ }^{218}$ Thus, it is essential to measure and prove the reliability of obtained data of $\mathrm{NH}_{3}$ amount. Suryanto, MacFarlane and co-workers ${ }^{194}$ summarized the current steps and mis-steps towards NRR in terms of experimental methodology and catalyst selection, and proposed a protocol for rigorous experimentation. They discussed the protocols of NRR experiments in detail and proposed a five-step experimental protocol including gas purification, open circuit control measurements and parallel control experiments by ${ }^{15} \mathrm{~N}$ isotopic labelling experiments to exclude the ammonia contamination or catalyst decomposition issues for reliable proof of the occurrence of the electrochemical nitrogen reduction reaction drawing on Greenlee and co-workers. ${ }^{216}$ A similar rigorous experimental protocol consisting of standardized control experiments and quantitative isotope measurements with ${ }^{15} \mathrm{~N}$ gas is also proposed by Tang and Qiao ${ }^{219}$ based on the Nature published, Chorkendorff and colleague's landmark study about the "true or false" issue in the electrochemical NRR research community in 2019..$^{20}$

In this regard, only with careful validation of the nitrogen source can we evaluate the NRR performance of potential 
Table 2 Summary of the performances of NRR electrocatalysts

\begin{tabular}{|c|c|c|c|c|c|}
\hline Electrocatalysts & Electrolyte & $\mathrm{NH}_{3}$ yield & $\mathrm{FE}(\%)$ & $\begin{array}{l}\text { Overpotential (V) } \\
\text { vs. RHE }\end{array}$ & Ref. \\
\hline \multicolumn{6}{|l|}{ Noble metals } \\
\hline Ag film & $0.1 \mathrm{M} \mathrm{Na}_{2} \mathrm{SO}_{4}$ & $1.27 \mu \mathrm{g} \mathrm{h}^{-1} \mathrm{~cm}^{-2}$ & 7.36 & -0.6 & 244 \\
\hline Ag nanosheets & $0.1 \mathrm{M} \mathrm{HCl}$ & $2.83 \mu \mathrm{g} \mathrm{h}^{-1} \mathrm{~cm}^{-2}$ & 4.8 & -0.6 & 232 \\
\hline Au flowers & $0.1 \mathrm{M} \mathrm{HCl}$ & $25.57 \mu \mathrm{g} \mathrm{h}^{-1} \mathrm{mg}_{\text {cat }}^{-1}$ & 6.05 & -0.2 & 351 \\
\hline $\mathrm{Au} / \mathrm{TiO}_{2}$ & $0.1 \mathrm{M} \mathrm{HCl}$ & $21.4 \mu \mathrm{g} \mathrm{h}^{-1} \mathrm{mg}^{-1}$ cat & 8.11 & -0.2 & 352 \\
\hline $\mathrm{a}-\mathrm{Au} / \mathrm{CeOx}-\mathrm{RGO}$ & $0.1 \mathrm{M} \mathrm{HCl}$ & $8.3 \mu \mathrm{g} \mathrm{h}^{-1} \mathrm{mg}^{-1}$ cat & 10.1 & -0.2 & 353 \\
\hline Porous Au film on Ni foam & $0.1 \mathrm{M} \mathrm{Na}_{2} \mathrm{SO}_{4}$ & $9.42 \mu \mathrm{g} \mathrm{h}^{-1} \mathrm{~cm}^{-2}$ & 13.36 & -0.2 & 226 \\
\hline $\mathrm{Pd} / \mathrm{C}$ & $0.1 \mathrm{M}$ PBS & $4.5 \mu \mathrm{g} \mathrm{h}^{-1} \mathrm{mg}^{-1}{ }_{\text {cat }}$ & 8.2 & 0.1 & 228 \\
\hline Rh NPs/C & $0.5 \mathrm{M} \mathrm{Na}_{2} \mathrm{SO}_{4}$ & $22.82 \pm 1.49 \mu \mathrm{g} \mathrm{h}^{-1} \mathrm{mg}^{-1}$ cat. & $\sim 0.1$ & -0.45 & 231 \\
\hline $\mathrm{Rh}_{2} \mathrm{Sb} \mathrm{RNRs} / \mathrm{C}$ & & $228.85 \pm 12.96 \mu \mathrm{g} \mathrm{h}^{-1} \mathrm{mg}^{-1}$ cat. & $\sim 1.5$ & & \\
\hline $\mathrm{Rh}_{2} \mathrm{Sb} \mathrm{SNRs} / \mathrm{C}$ & & $63.07 \pm 4.45 \mu \mathrm{g} \mathrm{h}^{-1} \mathrm{mg}^{-1}$ cat. & $\sim 0.4$ & & \\
\hline $\mathrm{Ru}_{1}$ on $\mathrm{N}$-doped carbon & $0.05 \mathrm{M} \mathrm{H}_{2} \mathrm{SO}_{4}$ & $120.9 \mu \mathrm{g} \mathrm{h}^{-1} \mathrm{mg}_{\text {cat }}^{-1}$ & 29.6 & -0.2 & 315 \\
\hline \multicolumn{6}{|l|}{ Non-noble metals } \\
\hline \multicolumn{6}{|l|}{ Metallic substances } \\
\hline Fe SA-N-C & $0.1 \mathrm{M} \mathrm{KOH}$ & $7.48 \mu \mathrm{g} \mathrm{h}^{-1} \mathrm{mg}_{\text {cat }}^{-1}$ & 56.55 & 0 & 354 \\
\hline $\mathrm{Mo}_{1}$ on $\mathrm{N}$-doped porous carbon & $0.1 \mathrm{M} \mathrm{KOH}$ & $34.0 \pm 3.6 \mu \mathrm{g} \mathrm{h}^{-1} \mathrm{mg}^{-1}$ cat. & $14.6 \pm 1.6$ & -0.3 & 355 \\
\hline \multicolumn{6}{|l|}{ Metal oxides } \\
\hline B-doped $\mathrm{TiO}_{2}$ & $0.1 \mathrm{M} \mathrm{Na}_{2} \mathrm{SO}_{4}$ & $14.4 \mu \mathrm{g} \mathrm{h}^{-1} \mathrm{mg}^{-1}$ cat. & 3.4 & -0.8 & 274 \\
\hline $\mathrm{C}_{-} \mathrm{TiO}_{2}$ nanoparticles & $0.1 \mathrm{M} \mathrm{Na}_{2} \mathrm{SO}_{4}$ & $16.22 \mu \mathrm{g} \mathrm{h}^{-1} \mathrm{mg}^{-1}$ cat. & 1.84 & -0.7 & 273 \\
\hline $\mathrm{Fe}_{2} \mathrm{O}_{3}$ & $1.0 \mathrm{M} \mathrm{KOH}$ & $0.46 \mu \mathrm{g} \mathrm{h}^{-1} \mathrm{~cm}^{-2}$ & 6.04 & -0.074 & 260 \\
\hline $\mathrm{Fe}_{2} \mathrm{O}_{3}$ nanorods & $0.1 \mathrm{M} \mathrm{Na}_{2} \mathrm{SO}_{4}$ & $15.9 \mu \mathrm{g} \mathrm{h}^{-1} \mathrm{mg}^{-1}$ cat. & 0.94 & -0.8 & 265 \\
\hline $\mathrm{Fe}_{2} \mathrm{O}_{3} / \mathrm{TiO}_{2}$ & $1.0 \mathrm{M} \mathrm{KOH}$ & $16.52 \mu \mathrm{g} \mathrm{h}^{-1} \mathrm{mg}^{-1}$ cat. & 0.31 & -0.577 & 356 \\
\hline $\mathrm{Nb}_{2} \mathrm{O}_{5}$ nanowire array & $0.1 \mathrm{M} \mathrm{Na}_{2} \mathrm{SO}_{4}$ & $9.67 \mu \mathrm{g} \mathrm{h}^{-1} \mathrm{~cm}^{-2}$ & 2.26 & -0.6 & 210 \\
\hline $\mathrm{NbO}_{2}$ nanoparticles & $0.05 \mathrm{M} \mathrm{H}_{2} \mathrm{SO}_{4}$ & $\begin{array}{l}11.6 \mu \mathrm{g} \mathrm{h}^{-1} \mathrm{mg}^{-1} \text { cat. } \\
(-0.65 \mathrm{~V} v s . \mathrm{RHE})\end{array}$ & $32(-0.6 \mathrm{~V}$ vs. RHE) & - & 208 \\
\hline $\mathrm{TiO}_{2}$ nanosheet array & $0.1 \mathrm{M} \mathrm{Na}_{2} \mathrm{SO}_{4}$ & $5.62 \mu \mathrm{g} \mathrm{h}^{-1} \mathrm{~cm}^{-2}$ & 2.5 & -0.7 & 283 \\
\hline $\mathrm{TiO}_{2}$-rGO & $0.1 \mathrm{M} \mathrm{Na}_{2} \mathrm{SO}_{4}$ & $15.13 \mu \mathrm{g} \mathrm{h}^{-1} \mathrm{mg}^{-1}$ cat. & 3.3 & -0.9 & 272 \\
\hline Defective $\mathrm{TiO}_{2}$ on $\mathrm{Ti}$ mesh & $0.1 \mathrm{M} \mathrm{HCl}$ & $7.59 \mu \mathrm{g} \mathrm{h}^{-1} \mathrm{~cm}^{-2}$ & 9.17 & -0.15 & 284 \\
\hline $\mathrm{Fe} / \mathrm{Fe}_{3} \mathrm{O}_{4}$ & $0.1 \mathrm{M}$ PBS & $0.19 \mu \mathrm{g} \mathrm{h}^{-1} \mathrm{~cm}^{-2}$ & 8.29 & -0.3 & 267 \\
\hline FeOOH QDs-GS & $0.1 \mathrm{M} \mathrm{LiClO}_{4}$ & $27.3 \mu \mathrm{g} \mathrm{h}^{-1} \mathrm{mg}^{-1}$ cat. & 14.6 & -0.4 & 262 \\
\hline \multicolumn{6}{|l|}{ Metal chalcogenides } \\
\hline $\mathrm{CoS}_{2} / \mathrm{NC}$ & $0.1 \mathrm{M} \mathrm{HCl}$ & $17.45 \mu \mathrm{g} \mathrm{h}^{-1} \mathrm{mg}^{-1}$ cat. & 4.6 & -0.15 & 357 \\
\hline $\mathrm{Fe}_{3} \mathrm{~S}_{4}$ & $0.1 \mathrm{M} \mathrm{HCl}$ & $75.4 \mu \mathrm{g} \mathrm{h}^{-1} \mathrm{mg}^{-1}$ cat. & 6.45 & -0.4 & 358 \\
\hline \multicolumn{6}{|l|}{ Metal carbides } \\
\hline $\mathrm{Mo}_{2} \mathrm{C}$ nanorod & $0.1 \mathrm{M} \mathrm{HCl}$ & $95.1 \mu \mathrm{g} \mathrm{h}^{-1} \mathrm{mg}^{-1}$ cat. & 8.13 & -0.3 & 294 \\
\hline $\mathrm{Mo}_{2} \mathrm{C} / \mathrm{C}$ & $0.5 \mathrm{M} \mathrm{LiClO}_{4}$ & $11.3 \mu \mathrm{g} \mathrm{h}^{-1} \mathrm{mg}^{-1}$ cat. & 7.8 & -0.3 & 277 \\
\hline $\mathrm{Ti}_{3} \mathrm{C}_{2} \mathrm{~T}_{x}(\mathrm{~T}=\mathrm{F}, \mathrm{OH})$ MXene nanosheets & $0.1 \mathrm{M} \mathrm{HCl}$ & $20.4 \mu \mathrm{g} \mathrm{h}^{-1} \mathrm{mg}^{-1}$ cat. & 9.3 & -0.4 & 270 \\
\hline \multicolumn{6}{|l|}{ Metal nitrides } \\
\hline $\begin{array}{l}\text { VN nanosheet array } \\
\text { VN }\end{array}$ & $\begin{array}{l}0.1 \mathrm{M} \mathrm{KUH} \\
0.1 \mathrm{M} \mathrm{HCl}\end{array}$ & $\begin{array}{l}34.83 \mu \mathrm{g} \mathrm{h}^{-1} \mathrm{mg}^{-2} \text { cat. } \\
5.14 \mu \mathrm{g} \mathrm{h}^{-1} \mathrm{~cm}^{-2}\end{array}$ & $\begin{array}{l}9.28 \\
2.25\end{array}$ & $\begin{array}{l}-0.2 \\
-0.5\end{array}$ & $\begin{array}{l}359 \\
297\end{array}$ \\
\hline VN nanoparticles & $0.05 \mathrm{M} \mathrm{H}_{2} \mathrm{SO}_{4}$ & $20.2 \mu \mathrm{g} \mathrm{h}^{-1} \mathrm{~cm}^{-2}$ & 6 & -0.1 & 278 \\
\hline \multicolumn{6}{|l|}{ Metal phosphides } \\
\hline $\mathrm{CoP} / \mathrm{CNs}$ & $0.1 \mathrm{M} \mathrm{Na}_{2} \mathrm{SO}_{4}$ & $48.9 \mu \mathrm{g} \mathrm{h}^{-1} \mathrm{mg}^{-1}$ cat. & 8.7 & -0.4 & 279 \\
\hline \multirow[t]{2}{*}{$\mathrm{Ni}_{2} \mathrm{P} / \mathrm{N}, \mathrm{P}-\mathrm{C}$} & $0.1 \mathrm{M} \mathrm{KOH}$ & $90.1 \mu \mathrm{g} \mathrm{h}^{-1} \mathrm{mg}^{-1}$ cat. & 19.82 & -0.2 & 280 \\
\hline & $0.1 \mathrm{M} \mathrm{HCl}$ & $34.4 \mu \mathrm{g} \mathrm{h}^{-1} \mathrm{mg}^{-1}$ cat. & 17.21 & - & \\
\hline $\mathrm{FeP}_{2} \mathrm{NP}-\mathrm{rGO}$ & $0.5 \mathrm{M} \mathrm{LiClO}_{4}$ & $35.26 \mu \mathrm{g} \mathrm{h}^{-1} \mathrm{mg}^{-1}$ cat. & 21.99 & -0.4 & 299 \\
\hline \multicolumn{6}{|l|}{ Metal-free electrocatalysts } \\
\hline Defect-rich carbon cloth & $\begin{array}{l}0.1 \mathrm{M} \mathrm{Na}_{2} \mathrm{SO}_{4} \\
0.02 \mathrm{M} \mathrm{H}_{2} \mathrm{SO}_{4}\end{array}$ & $15.9 \mu \mathrm{g} \mathrm{h}^{-1} \mathrm{~cm}^{-2}$ & 6.92 & -0.3 & 304 \\
\hline N-Doped highly disordered carbon & $0.1 \mathrm{M} \mathrm{KOH}$ & $57.8 \mu \mathrm{g} \mathrm{h}^{-1} \mathrm{~cm}^{-2}$ & 10.2 & -0.3 & 310 \\
\hline $\mathrm{N}$ and B co-doped carbon nanosheets & $0.1 \mathrm{M} \mathrm{HCl}$ & $7.75 \mu \mathrm{g} \mathrm{h}^{-1} \mathrm{mg}^{-1}$ cat. & 13.79 & -0.3 & 326 \\
\hline Oxygen-doped hollow carbon microtubes & $0.1 \mathrm{M} \mathrm{HCl}$ & $25.12 \mu \mathrm{g} \mathrm{h}^{-1} \mathrm{mg}^{-1}$ cat. & 9.1 & -0.8 & 324 \\
\hline Oxygen-doped carbon nanosheet & $0.1 \mathrm{M} \mathrm{HCl}$ & $20.15 \mu \mathrm{g} \mathrm{h}^{-1} \mathrm{mg}^{-1}$ & 4.97 & -0.6 & 300 \\
\hline Polymeric carbon nitride & $0.1 \mathrm{M} \mathrm{HCl}$ & $8.09 \mu \mathrm{g} \mathrm{h}^{-1} \mathrm{mg}^{-1}$ cat. & 11.59 & -0.2 & 311 \\
\hline S-Doped carbon nanospheres & $0.1 \mathrm{M} \mathrm{Na}_{2} \mathrm{SO}_{4}$ & $19.07 \mu \mathrm{g} \mathrm{h}^{-1} \mathrm{mg}^{-1}$ cat. & 7.47 & -0.7 & 360 \\
\hline P-Doped graphene & $0.5 \mathrm{M} \mathrm{LiClO}_{4}$ & $32.33 \mu \mathrm{g} \mathrm{h}^{-1} \mathrm{mg}^{-1}$ cat & 20.82 & -0.65 & 306 \\
\hline S-Doped graphene & $0.1 \mathrm{M} \mathrm{HCl}$ & $\begin{array}{l}27.3 \mu \mathrm{g} \mathrm{h}^{-1} \mathrm{mg}^{-1} \text { cat. } \\
(-0.8 \mathrm{~V} v s . \mathrm{RHE})\end{array}$ & $11.5(-0.6 \mathrm{~V} v s . \mathrm{RHE})$ & - & 274 \\
\hline \multicolumn{6}{|l|}{ Elementals and their compounds } \\
\hline B-Doped graphene & $0.05 \mathrm{M} \mathrm{H}_{2} \mathrm{SO}_{4}$ & $9.8 \mu \mathrm{g} \mathrm{h}^{-1} \mathrm{~cm}^{-2}$ & 10.8 & -0.5 & 316 \\
\hline $\mathrm{B}_{4} \mathrm{C} / \mathrm{CPE}$ & $0.1 \mathrm{M} \mathrm{HCl}$ & $26.57 \mu \mathrm{g} \mathrm{h}^{-1} \mathrm{mg}^{-1}$ cat. & 15.95 & -0.75 & 328 \\
\hline Boron nitride (mesoporous) & $0.1 \mathrm{M} \mathrm{Na}_{2} \mathrm{SO}_{4}$ & $18.2 \mu \mathrm{g} \mathrm{h}^{-1} \mathrm{mg}^{-1}$ cat. & 5.5 & -0.7 & 314 \\
\hline Black phosphorus nanosheets & $0.01 \mathrm{M} \mathrm{HCl}$ & $\begin{array}{l}31.37 \mu \mathrm{g} \mathrm{h}^{-1} \mathrm{mg}^{-1} \text { cat. } \\
(-0.7 \mathrm{~V} v s . \mathrm{RHE})\end{array}$ & $5.07(-0.6 \mathrm{~V} v s . \mathrm{RHE})$ & - & 330 \\
\hline
\end{tabular}

catalysts. Several key parameters related to the performance of the NRR, including electrolyte, ammonia formation rate $\left(\mathrm{NH}_{3}\right.$ yield), faradaic efficiency and overpotential (V) vs. RHE of reported catalysts for NRR, are listed in Table 2 . It should be 
pointed out that since the accuracy of the determinate NRR efficiency highly relies on the meticulous measurement of $\mathrm{NH}_{3}$ amount and well controlled NRR experiments, it is necessary to refer to the original work and confirm the experimental protocols. A second party inspection of the results by using a well-accepted standard protocol will be helpful to filtrate the catalysts with reliable efficiency.

Furthermore, among the various research advances in electrochemical NRR, lithium-mediated nitrogen reduction has attracted much interest as it has been proven to be a good method to electrochemically synthesize ammonia in the past several years. ${ }^{221}$ The lithium-mediated nitrogen electroreduction also demonstrated good reproducibility ${ }^{219}$ but the process has so far been unstable, and the continuous deposition of lithium limits its practical applicability. The underlying mechanism of lithium-mediated NRR needs to be further investigated to ultimately contribute to green ammonia production and a sustainable society. Hence, the development of electrocatalysts for this purpose is crucial towards an effective NRR with high $\mathrm{NH}_{3}$ yield.

\subsubsection{Noble metal-based electrocatalysts}

Au-Based electrocatalysts. Noble metal-based materials, as effective NRR electrocatalysts, have the potential for surficial interaction with $\mathrm{N}_{2}$ through electron transfer, which is required to promote $\mathrm{N}_{2}$-fixation. ${ }^{22}$ Gold ( $\mathrm{Au}$ )-based materials, having one of the most stable metallic configurations, are targeted as NRR electrocatalysts owing to their low HER activity. Under this circumstance, electrochemical fixation of $\mathrm{N}_{2}$ is promoted, ${ }^{223}$ although theoretical studies dispute this claim. ${ }^{206,224}$ Yan and co-workers demonstrated the potential of a Au-based material (tetrahexahedral (THH) Au nanorods) as a heterogeneous NRR electrocatalyst under ambient conditions. Here, a stepped (730) facet (consisting of (210) and (310) sub-facets) of THH $\mathrm{Au}$ nanorods at room temperature and pressure yielded $1.648 \mu \mathrm{g} \mathrm{h}^{-1} \mathrm{~cm}^{-2}$ of $\mathrm{NH}_{3}$ and $0.102 \mu \mathrm{g} \mathrm{h} \mathrm{cm}^{-1}$ of $\mathrm{N}_{2} \mathrm{H}_{4} \cdot \mathrm{H}_{2} \mathrm{O}$ with a high FE (4.02\%) at $-0.2 \mathrm{~V}$ (vs. RHE) (Fig. $5(\mathrm{a})$ and (b)). ${ }^{223}$ It was suggested that the stepped facets outperformed the flat planes due to the availability of more catalyst active sites for the adsorption and activation of $\mathrm{N}_{2}{ }^{225}$

To promote access to more active sites and enhance the $\mathrm{NH}_{3}$ yield rate and selectivity, porous Au materials are suggested. ${ }^{226}$ Wang and co-workers employed a micelle-assisted electrodeposition approach to directly synthesize a porous $\mathrm{Au}$ film on $\mathrm{Ni}$ foam as an NRR electrocatalyst. In $0.1 \mathrm{M} \mathrm{Na}_{2} \mathrm{SO}_{4}$ under ambient conditions, the porous Au film presented an $\mathrm{NH}_{3}$ yield rate of $9.42 \mu \mathrm{g} \mathrm{h}^{-1} \mathrm{~cm}^{-2}$ and an FE of $13.36 \%$ at $-0.2 \mathrm{~V}$ (vs. RHE). ${ }^{226}$ Nazemi and co-workers engineered hollow Au nanocages (Fig. 5(c)) of various pore size/density and $\mathrm{Au}$ content for the electrochemical synthesis of $\mathrm{NH}_{3}$. It was demonstrated that the $715 \mathrm{~nm}$ pore size (38.3 Au-wt\%) displayed the optimal performance with an $\mathrm{NH}_{3}$ yield rate of $3.9 \mu \mathrm{g} \mathrm{cm} \mathrm{ch}^{-2} \mathrm{~h}^{-1}$ and a large $\mathrm{FE}$ of $30.2 \%$ (Fig. 5(d)). The observed improvement in catalytic activity is attributable to the nanoscale confinement of $\mathrm{N}_{2}$ near the catalyst surface. ${ }^{227}$

Pd-Based electrocatalysts. Nowadays, neat palladium (Pd) has been argued to be not suitable as a catalyst for $\mathrm{N}_{2}-\mathrm{to}-\mathrm{NH}_{3}$ conversion owing to the preferential affinity for hydrogen than nitrogen resulting in the hindrance of $\mathrm{N}_{2}$ adsorption. ${ }^{30}$ To circumvent this, strategic schemes to suppress the HER are advised. One of the strategies is adopting a hydrogen spillover phenomenon whereby the $\mathrm{H}$-species are adsorbed on the catalyst and then spilled over to the adsorbed $\mathrm{N}_{2}$ for hydrogenation to occur. By doing so, the kinetics for $\mathrm{N}_{2}-$ to- $\mathrm{NH}_{3}$ conversion will be accelerated. Accordingly, hydrides were suggested with the potential of boosting surface $\mathrm{N}_{2}$ hydrogenation via a hydrogen transfer mechanism. ${ }^{234,235}$ However, the formation of Pd-hydrides requires the use of highly reducing agents which could result in difficult formation of hydrides. ${ }^{236}$ In this case, studies have investigated the use of electrides as an alternative approach. Electrides are ionic compounds known for their ease of electron donation. Under this circumstance, their usage will provide electrons for the adsorbed $\mathrm{N}_{2}$ while trapping excess electrons to produce the hydride.

Xin and co-workers highlighted this effect using Pd nanoparticles on a carbon black support $(\mathrm{Pd} / \mathrm{C})$, which can generate Pd-hydrides under specific potentials (Fig. $5(\mathrm{e})$ and (f)). ${ }^{228}$ This mechanism allowed for the effective suppression of HER in $0.1 \mathrm{M}$ PBS and hence facilitated the Grotthuss-like hydride transfer mechanism on $\alpha-\mathrm{PdH}$ for the hydrogenation of $\mathrm{N}_{2}$. The beneficial effect of PBS in promoting $\mathrm{N}_{2}$ hydrogenation at $-0.05 \mathrm{~V}$ (vs. RHE) (yield rate $=4.9 \mu \mathrm{g} \mathrm{h}^{-1} \mathrm{mg}^{-1}$ Pd) is twice the yield from $0.05 \mathrm{M} \mathrm{H}_{2} \mathrm{SO}_{4}\left(2.5 \mu \mathrm{g} \mathrm{h}^{-1} \mathrm{mg}^{-1}{ }_{\mathrm{Pd}}\right)$ and $0.1 \mathrm{M}$ $\mathrm{NaOH}\left(2.1 \mu \mathrm{g} \mathrm{h}^{-1} \mathrm{mg}^{-1}{ }_{\mathrm{Pd}}\right)$. The controlled potential electrolysis on the $\mathrm{Pd} / \mathrm{C}$ nanoparticles resulted in an $\mathrm{NH}_{3}$ yield rate of $\sim 4.5 \mu \mathrm{g} \mathrm{h}^{-1} \mathrm{mg}^{-1}$ Pd and a FE of $8.2 \%$ at $0.1 \mathrm{~V}$ (vs. RHE) (at a low overpotential of $56 \mathrm{mV}$ ), outperforming $\mathrm{Au}$ and $\mathrm{Pt}$ catalysts. $^{228}$

Moreover, Pd catalysts can be modified for $\mathrm{N}_{2}$-to- $\mathrm{NH}_{3}$ transformation by integrating with other metals to produce alloys. For highly effective catalyst systems, Jacobsen and co-workers proposed the rational approach of forming alloys with elements from the different sides of the volcano plot. Specifically, integrating a metal with strong $\mathrm{N}_{2}$ affinity with another with weak affinity is more likely to yield optimum ammonia synthesis. ${ }^{237}$ On this account, Yan and co-workers developed an amorphous $\mathrm{PdCu}$ nanocluster on $\mathrm{rGO}$ for catalyzing $\mathrm{NH}_{3}$ synthesis ${ }^{229}$ based on the characteristics of amorphous $\mathrm{Cu}$ to promote the hydrogen-spillover mechanism. ${ }^{238}$ In addition to the excellent electron transport property of rGO, this support promotes the dispersion and even distribution of the alloy nanoparticles, thereby preventing particle agglomeration (Fig. 5(g)). Based upon the above principle, the optimal $\mathrm{Pd}_{0.2} \mathrm{Cu}_{0.8}$ alloy nanoparticles significantly outperformed the individual components and displayed an $\mathrm{NH}_{3}$ yield rate of $2.80 \mu \mathrm{g} \mathrm{h}^{-1} \mathrm{mg}^{-1}$ cat. with a low $\mathrm{FE}$ of about $0.8 \%$ at $-0.2 \mathrm{~V}$ (vs. RHE) in $0.1 \mathrm{M} \mathrm{KOH}$ (Fig. $5(\mathrm{~h}))^{229}$

Ru-Based electrocatalysts. Ruthenium (Ru) is an exceptional catalyst for the synthesis of $\mathrm{NH}_{3}$ via the conventional HaberBosch process. ${ }^{239}$ Theoretically, it is revealed that Ru displays a lower $\mathrm{N}_{2}$ adsorption energy and overpotential under both associative and dissociative mechanisms than other noble metals in the electrocatalytic synthesis of $\mathrm{NH}_{3}$. Similar to other 


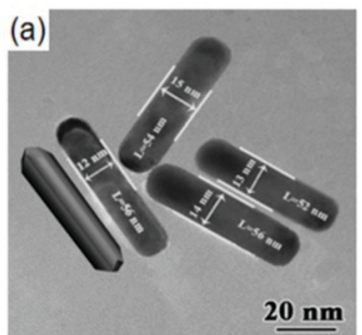

(b)
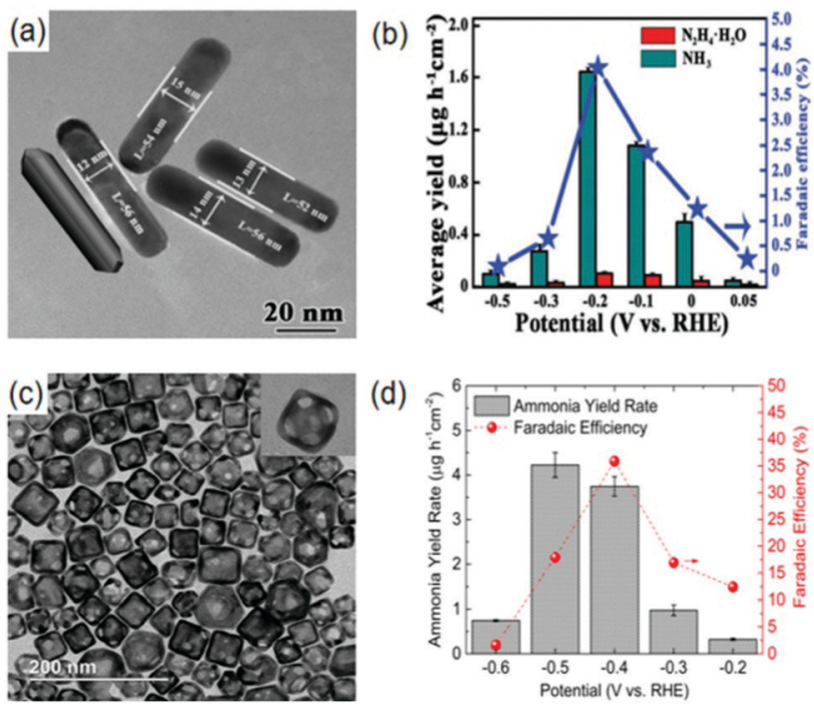

(d)

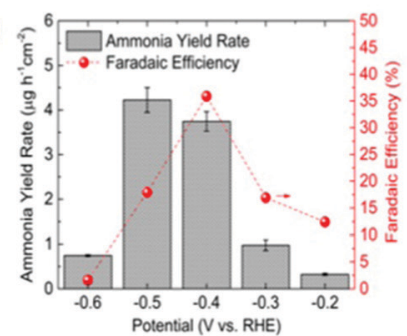

(f)

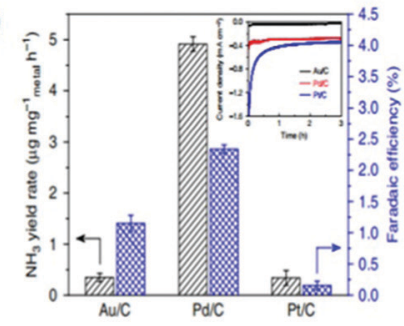

(h)
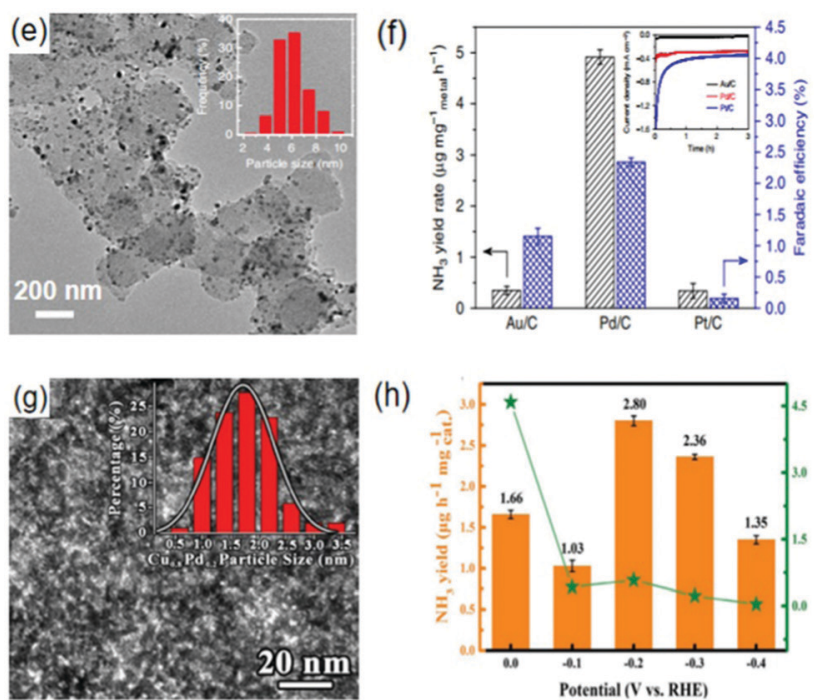
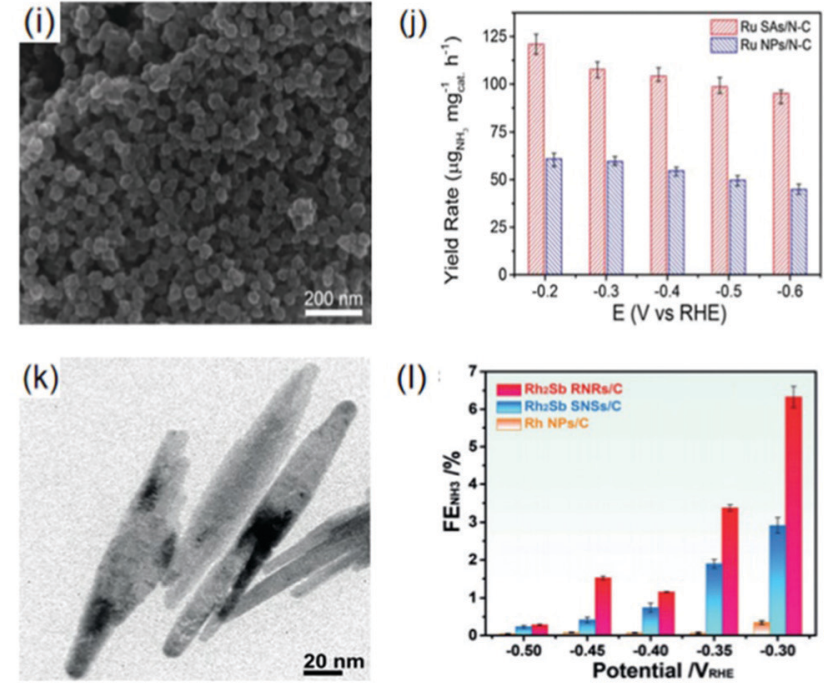

(I)
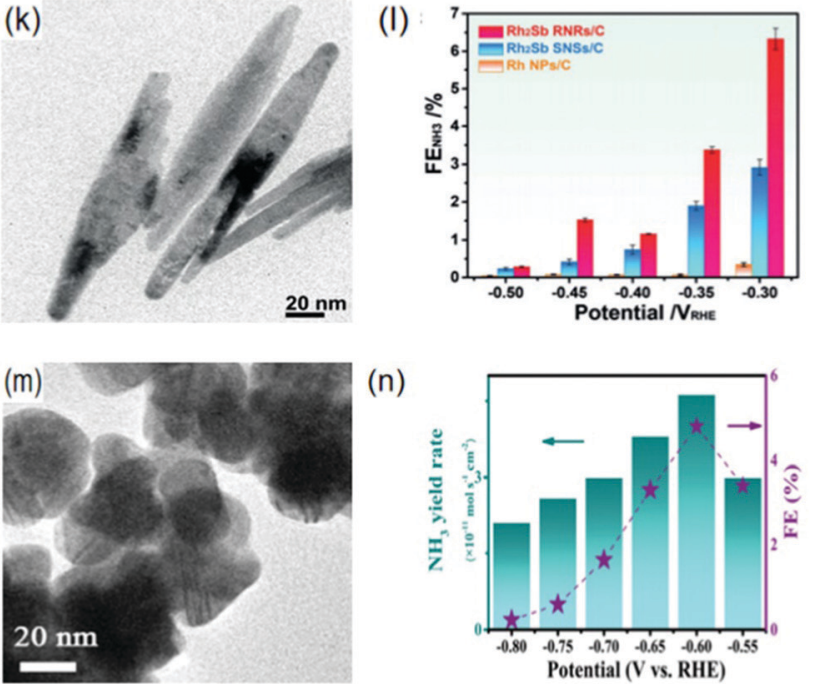

(n)
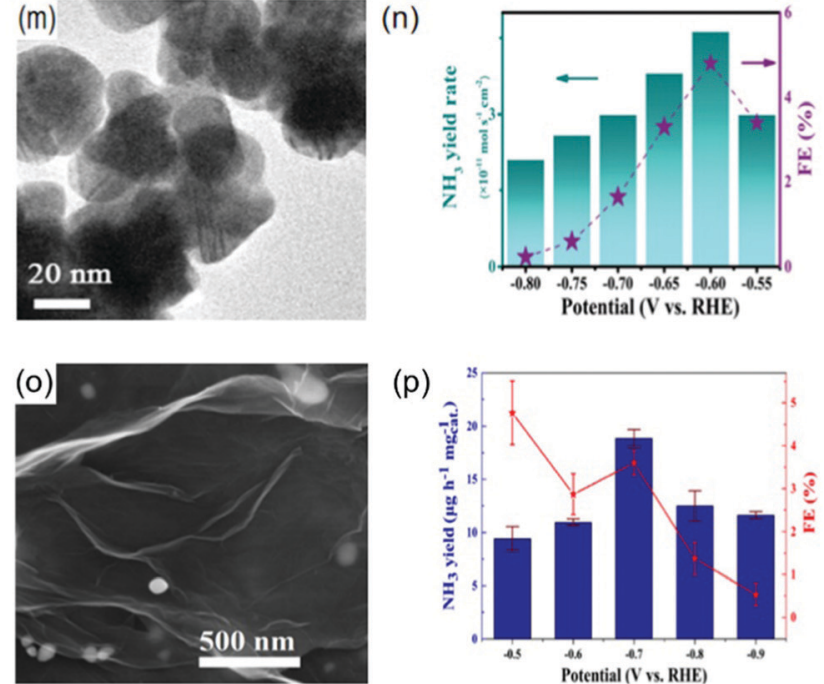

(p)

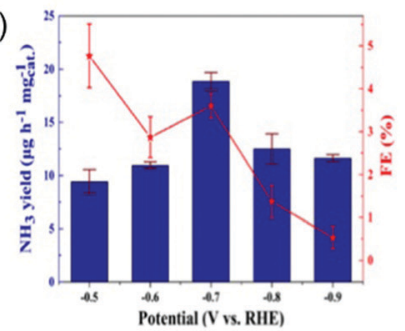

Fig. 5 (a) TEM image of Au nanorods with aspect ratio $4 \pm 0.5$. (b) Yield rate of ammonia (cyan), hydrazine hydrate (red) formation, and faradaic efficiency (blue) at each given potential. Panels (a) and (b) are reproduced with permission. ${ }^{223}$ Copyright 2017, Wiley. (c) TEM image of hollow Au nanocages, and their (d) $\mathrm{NH}_{3}$ yield rate and $\mathrm{FE}$ at different potentials in $0.5 \mathrm{M} \mathrm{LiClO}_{4}$ aqueous solution. Panels (c) and (d) are reproduced with permission. ${ }^{227}$ Copyright 2018, American Chemical Society. (e) TEM image of the Pd/C catalyst (inset: particle size distribution). (f) $\mathrm{NH} 33$ yield rates and FE of the $\mathrm{Pd} / \mathrm{C}$ catalyst in $0.05 \mathrm{M} \mathrm{H}_{2} \mathrm{SO}_{4}, 0.1 \mathrm{M} \mathrm{PBS}$ and $0.1 \mathrm{M} \mathrm{NaOH}$ at $-0.05 \mathrm{~V}$ (vs. RHE). Panels (e) and (f) are reproduced with permission. ${ }^{228} \mathrm{Copyright}$ 2018, Nature Communication. (g) STEM image of $\mathrm{Pd}_{0.2} \mathrm{Cu}_{0.8} / \mathrm{rGO}$ (inset: particle size distribution). (h) $\mathrm{NH}_{3}$ yield rates and $\mathrm{FE}$ of the $\mathrm{Pd}_{0.2} \mathrm{Cu}_{0.8} / \mathrm{rGO}$ composite at different potentials in $0.1 \mathrm{M} \mathrm{KOH}$. Panels (g) and (h) are reproduced with permission. ${ }^{229}$ Copyright 2018, Wiley. (i) SEM image of Ru SAs/ $\mathrm{N}$ - C. (j) yield rate of $\mathrm{NH}_{3}$ production at different applied potentials on Ru SAs/N-C and Ru NPs/N-C. Panels (i) and (j) are reproduced with permission. ${ }^{230}$ Copyright 2018, Wiley. (k) TEM image of $\mathrm{Rh}_{2} \mathrm{Sb}$ SNRs. (l) FE in comparison to $\mathrm{Rh}_{2} \mathrm{Sb} \mathrm{RNRs} / \mathrm{C}, \mathrm{Rh}_{2} \mathrm{Sb} \mathrm{SNRs} / \mathrm{C}$, and Rh NPs/C at different potentials. Panels $(\mathrm{k})$ and $(\mathrm{l})$ are reproduced with permission. ${ }^{231}$ Copyright 2020, Wiley. $(\mathrm{m})$ TEM image of the Ag nanosheet, and its corresponding (n) $\mathrm{NH}_{3}$ yield rate and $\mathrm{FE}$ at different potentials. Panels $(\mathrm{m})$ and $(\mathrm{n})$ are reproduced with permission. ${ }^{232}$ Copyright 2018, Royal Society of Chemistry. (o) SEM image of Ag NPs-rGO, and its corresponding (p) $\mathrm{NH}_{3}$ yield rate and FE at different potentials. Panels (o) and (p) are reproduced with permission. ${ }^{233}$ Copyright 2020 , Springer Nature Switzerland AG. Part of Springer Nature.

electrocatalysts, the HER impedes the NRR by decreasing the number of catalyst active sites due to the increased ${ }^{*} \mathrm{H}$ coverage. This fact considerably affects the energy barrier for the first $\mathrm{N}_{2}$ hydrogenation and desorption of ${ }^{*} \mathrm{NH}_{2} \cdot{ }^{240}$ One of such modalities to promote the NRR activity of Ru-based electrocatalysts is the anchoring of $\mathrm{Ru}$ atoms on appropriate support structures such as N-doped carbon. For instance, Geng and co-workers demonstrated that $\mathrm{Ru}$ atoms anchored on
$\mathrm{N}$-doped carbon ( $\mathrm{Ru}$ SAs/N-C) attained a remarkably high $\mathrm{NH}_{3}$ yield rate of $120.9 \mu \mathrm{g} \mathrm{h}^{-1} \mathrm{mg}^{-1}$ cat. and $29.6 \% \mathrm{FE}$ (Fig. 5(i) and (j)). ${ }^{230}$ The reason behind this is that with the aid of the porous and defective $\mathrm{N}$-doped carbon, the dispersion of isolated $\mathrm{Ru}$ atoms throughout the $\mathrm{Ru} \mathrm{SAs} / \mathrm{N}-\mathrm{C}$ structure promoted the adequate coordination of $\mathrm{Ru}$ atoms by $\mathrm{N}$ atoms. Similarly, atomic $\mathrm{Ru}$ doped in $\mathrm{Mo}_{2} \mathrm{CTX}$ MXene remarkably promoted the electrochemical $\mathrm{N}_{2}$-to- $\mathrm{NH}_{3}$ conversion. ${ }^{241}$ 
Rh-Based electrocatalysts. Based on several theoretical investigations, rhodium (Rh) can be considered as a promising NRR electrocatalyst as indicated by its position at the top of the volcano plot. So far, experimental studies have demonstrated various Rh nanostructures to be suitable for the electroreduction of $\mathrm{N}_{2}$ to $\mathrm{NH}_{3}$. This includes structures such as nanosheets, ${ }^{242}$ nanoparticles, ${ }^{231}$ nanowires ${ }^{243}$ and nanorods. ${ }^{231}$ For instance, Hou and co-workers recently reported that ultrathin $\mathrm{Rh}$ nanosheet nanoassemblies (Rh NNs) demonstrated an excellent NRR catalytic activity with a yield rate of $23.88 \mu \mathrm{g} \mathrm{h}^{-1} \mathrm{mg}^{-1}$ cat at $-0.2 \mathrm{~V}$ (vs. RHE). Moreover, $\mathrm{Rh}$ NNs displayed good $\mathrm{NH}_{3}$ selectivity without the formation of $\mathrm{N}_{2} \mathrm{H}_{4}$. The remarkable activity is attributed to the unique ultrathin two-dimensional nanosheet structure (ca. $1 \mathrm{~nm}$ ), modified electronic construction and high SSA. $^{242}$

To further improve the catalytic activity, schemes to modulate the surface roughness of the catalysts were considered. Adopting a facile hydrothermal approach, Zhang and co-workers synthesized a surface-rough $\mathrm{Rh}_{2} \mathrm{Sb}$ nanorod on carbon $\left(\mathrm{Rh}_{2} \mathrm{Sb}\right.$ RNRs/C) and compared its NRR performance with that of a surface-smooth $\mathrm{Rh}_{2} \mathrm{Sb}$ nanorod $\left(\mathrm{Rh}_{2} \mathrm{Sb} \mathrm{SNRs} / \mathrm{C}\right)$ and $\mathrm{Rh}$ NPs/C. The $\mathrm{NH}_{3}$ yield rates attained by these catalysts are $228.85 \pm$ $12.96 \mu \mathrm{g} \mathrm{h}^{-1} \mathrm{mg}_{\mathrm{Rh}}^{-1}, 63.07 \pm 4.45 \mu \mathrm{g} \mathrm{h}^{-1} \mathrm{mg}^{-1}{ }_{\mathrm{Rh}}$ and $22.82 \pm$ $1.49 \mu \mathrm{g} \mathrm{h}^{-1} \mathrm{mg}_{\mathrm{Rh}}^{-1}$, respectively, at $-0.45 \mathrm{~V}$ (vs. RHE), with $10 \mathrm{~h}$ stability witnessed by $\mathrm{Rh}_{2} \mathrm{Sb}$ RNRs/C (Fig. 5(k) and (l)). ${ }^{231}$ The superior catalytic activity by $\mathrm{Rh}_{2} \mathrm{Sb}$ RNRs was attributed to the high-index facets which enhanced the adsorption and activation of $\mathrm{N}_{2}$.

Ag-Based electrocatalysts. Among all the noble metals utilized as catalyst systems, silver $(\mathrm{Ag})$ is the most promising candidate given that it is more abundant and offers the lowest cost relative to its catalytic activities. On this account, inquiry into its catalytic ability in the electrochemical synthesis of $\mathrm{NH}_{3}$ has gained some attention. Our group conducted a pioneering study in this regard and revealed that $\mathrm{Ag}$ nanosheets are an efficient electrocatalyst for $\mathrm{N}_{2}$ fixation to $\mathrm{NH}_{3}$ with remarkable stability and selectivity under ambient conditions. When tested in $0.1 \mathrm{M} \mathrm{HCl}$, the $\mathrm{Ag}$ nanosheets achieved a yield rate of $4.62 \times$ $10^{-11} \mathrm{~mol} \mathrm{~s}^{-1} \mathrm{~cm}^{-2}$ and $4.8 \% \mathrm{FE}$ at $-0.60 \mathrm{~V}$ ( $v s$. RHE) with $24 \mathrm{~h}$ stability (Fig. 5(m) and (n)). ${ }^{232}$

To further improve the NRR efficiency, measures to retard the HER are necessary. On this account, Ji and co-workers revealed that the adsorption of halide anions on the surface of porous Ag effectively assisted the suppression of HER. In this study, the authors fabricated a nanoporous bromide-derived Ag film on $\mathrm{Ag}$ foil (BD-Ag/AF) with adsorbed $\mathrm{Br}$ - anions by means of in situ electrochemical reduction of the $\mathrm{AgBr}$ film on $\mathrm{Ag}$ foil. When tested in $0.1 \mathrm{M} \mathrm{Na}_{2} \mathrm{SO}_{4}, \mathrm{BD}-\mathrm{Ag} / \mathrm{AF}$ attained an improved FE efficiency of $7.36 \%$ in comparison to $0.38 \%$ of the porous $\mathrm{Ag}$ film alone, and a yield rate of $2.07 \times 10^{-11} \mathrm{~mol} \mathrm{~s}^{-1} \mathrm{~cm}^{-2}$ at $-0.6 \mathrm{~V}$ (vs. RHE) with $20 \mathrm{~h}$ stability. ${ }^{244}$

Despite the remarkable stability, the harsh self-aggregation of small-sized $\mathrm{Ag}$ nanoparticles affects their activity in addition to decreasing the electronic conductivity. To this effect, employing conductive substrates such as RGO to boost the catalyst's conductive features while simultaneously enabling particle dispersion has been proven to enhance the activity of the $\mathrm{N}_{2}$ to- $\mathrm{NH}_{3}$ conversion. For instance, $\mathrm{Li}$ and co-workers fabricated a Ag nanoparticles-reduced graphene oxide hybrid (Ag NPs-rGO) as a high-efficiency electrocatalyst for the NRR. When tested in $0.1 \mathrm{M} \mathrm{Na}_{2} \mathrm{SO}_{4}, \mathrm{Ag}$ NPs-rGO achieved an $\mathrm{NH}_{3}$ yield rate of $18.86 \mu \mathrm{g} \mathrm{h}^{-1} \mathrm{mg}^{-1}$ cat. and $3.60 \% \mathrm{FE}$ at $-0.7 \mathrm{~V}$ ( $v s$. RHE) (Fig. 5(o) and (p)), outperforming the Ag NPs under the same conditions (yield $=9.43 \mu \mathrm{g} \mathrm{h}^{-1} \mathrm{mg}^{-1}$ cat. and $\mathrm{FE}=2.25 \%$ ). ${ }^{233}$

\subsubsection{Non-noble metal based electrocatalysts}

Metallic substance. Similar to noble metal based electrocatalysts, non-noble metals and their compounds have also shown good NRR activity and are considered as potential replacements for noble metal electrocatalysts. Molybdenum (Mo) is one of the many metallic elements with a strong affinity for $\mathrm{N}_{2}$ adsorption, which is a preliminary characteristic for the NRR. The (110)-oriented Mo nanofilm has been proven to be an efficient catalyst under ambient conditions. Electrochemically, the (110)-oriented Mo nanofilm yielded $\mathrm{NH}_{3}$ at a rate of $1.89 \mu \mathrm{g} \mathrm{h}^{-1} \mathrm{~cm}^{-2}$ and an $\mathrm{FE}$ of $0.72 \%$ at $-0.49 \mathrm{~V}$ (vs. RHE) and $0.14 \mathrm{~V}$ (vs. RHE), respectively. ${ }^{245}$ The low $\mathrm{NH}_{3}$ yield was attributed to the strong Mo-N bonding making it difficult for the desorption of $\mathrm{NH}_{3}$. To circumvent this, it is recommended to introduce higher electronegative non-metal atoms (such as $\mathrm{N}$ ) into the Mo lattice which could potentially assist the reduction of electron charge transfer between the Mo and N-adatoms/admolecules. ${ }^{205,246}$

Because of the unique electronic structure and sluggish HER activity, bismuth (Bi) based materials demonstrate a high NRR performance. The ammonia yield of the fragmented $\mathrm{Bi}^{0}$ nanoparticles was found to be $3.25 \pm 0.08 \mu \mathrm{g} \mathrm{cm} \mathrm{cm}^{-2} \mathrm{~h}^{-1}$ at $-0.7 \mathrm{~V} v$ s. RHE with a faradaic efficiency of $12.11 \pm 0.84 \%$ at $-0.6 \mathrm{~V} v$ s. RHE. ${ }^{247}$ Compared with $\mathrm{Bi}^{0}$ nanoparticles, the three dimensional amorphous BiNi alloy showed an enhanced NRR activity. The $\mathrm{NH}_{3}$ yield rate of this structure was $17.5 \mu \mathrm{g} \mathrm{h}{ }^{-1} \mathrm{mg}_{\text {cat }}{ }^{-1}$ with a faradaic efficiency of $13.8 \%$ at $-0.6 \mathrm{~V} v s$. RHE. These two works indicate the significance of the electronic and geometric structure of the electrocatalysts in NRR. ${ }^{248}$

Another highly desirable metal material for the electrochemical $\mathrm{N}_{2}$-to- $\mathrm{NH}_{3}$ conversion is iron (Fe) given its important role as a catalyst system. ${ }^{249} \mathrm{~A}$ typical example is its function as an earth-abundant and low-cost catalyst for $\mathrm{NH}_{3}$ production in the industrial Haber-Bosch process. ${ }^{250}$ In terms of biological $\mathrm{N}_{2}$ fixation, $\mathrm{Fe}$ is also present in all three forms of nitrogenase enzymes (MoFe-, VFe-, and FeFe-nitrogenase). ${ }^{251}$ Founded on this, several investigative studies have focused on developing Fe-based catalyst systems that can support the electrosynthesis of $\mathrm{NH}_{3}$. First, theoretical evidence has revealed that $\mathrm{Fe}$ is one of the most promising NRR electrocatalysts among the available TMs. $^{201}$ Further studies have demonstrated the associated mechanism as the NRR pathway on Fe-based electrocatalysts such as $\mathrm{Fe}_{2} \mathrm{O}_{3}$ with the first protonation step being the ratedetermining step. ${ }^{252}$ Similar to all other metallic substances, Fe-based metal electrocatalysts display low $\mathrm{NH}_{3}$ yield resulting from passivated electrocatalytic activity from aggregated Fespecies generated during the NRR. ${ }^{202,203}$ 
Metal hydroxides and oxides. As previously mentioned, Fe has shown a tremendous application as a catalyst system. Likewise, iron oxide based materials such as $\mathrm{Fe}_{2} \mathrm{O}_{3}$ have also presented good catalytic characteristics. ${ }^{253,254}$ Experimental studies on $\mathrm{Fe}_{2} \mathrm{O}_{3}$ NPs over a Ni cathode have demonstrated a substantial $\mathrm{NH}_{3}$ yield rate with a FE of nearly $35 \%$, but at a high temperature of $200{ }^{\circ} \mathrm{C}^{253}$ Moreover, it was established that the yield rate of $\mathrm{NH}_{3}$ is dependent on the $\mathrm{Fe}_{2} \mathrm{O}_{3}$ NPs. For instance, over a 100fold increase in yield was witnessed within the same time frame when the $\mathrm{Fe}_{2} \mathrm{O}_{3}$ particle size was reduced from $\sim 70 \mu \mathrm{m}$ to 1-3 $\mu \mathrm{m}$. However, for a further decrease to 10-30 nm, the NRR was too rapid and violent to be evaluated. ${ }^{254}$

In addition, the use of conductive supports has also demonstrated to improve the catalytic utilization of $\gamma-\mathrm{Fe}_{2} \mathrm{O}_{3}$ NPs. Anchoring $\gamma-\mathrm{Fe}_{2} \mathrm{O}_{3}$ NPs on porous CP resulted in an $\mathrm{NH}_{3}$ yield rate of $0.9503 \mu \mathrm{g} \mathrm{h}^{-1} \mathrm{mg}^{-1},{ }^{255}$ which is triple the yield from the Fe catalyst $\left(0.3044 \mu \mathrm{g} \mathrm{h}^{-1} \mathrm{mg}^{-1}\right) \cdot{ }^{188}$ Likewise with other TMs, the enhanced activity is related to the improved interface between the NPs and the carbon surface, which offers unique carbon sites for $\mathrm{N}_{2}$ fixation. ${ }^{256}$ Moreover, modulating the loading of the catalyst content on the support is suggested for optimal tuning of the $\mathrm{NH}_{3}$ formation rate. ${ }^{257}$

The nature of the electrolyte is another factor of great importance for the effective performance of NRR electrocatalysts. Generally, $\mathrm{N}_{2}$ fixation in a molten salt is associated with high temperatures requiring a high energy input. On this account, investigative studies have demonstrated the catalytic efficacy of Fe-based catalysts in different electrolytes such as $\mathrm{N}_{2}$ saturated ionic liquids ([C4mpyr], $[\mathrm{eFAP}]$ and $\left.\left[\mathrm{P}_{6,6,6,14}\right][\mathrm{eFAP}]\right)$ (Fig. 6(a) and (b) $)^{258}$ or $\mathrm{N}_{2}$-saturated alkaline electrolytes (Fig. 6(c) and (d)). ${ }^{255}$ In these studies, the high solubility of $\mathrm{N}_{2}$ in the ionic liquids enabled the high NRR activity.

The reaction of $\mathrm{Fe}_{2} \mathrm{O}_{3}$ in an alkaline aqueous solution is represented by reaction (41). ${ }^{259}$ In this case, the generated $\mathrm{Fe}(\mathrm{OH})_{2}$ could lead to the passivation of $\mathrm{Fe}_{2} \mathrm{O}_{3}$, which affects the activity on the surface of $\mathrm{Fe}_{2} \mathrm{O}_{3} \cdot{ }^{260}$ However, based on reaction (42), $\mathrm{Fe}(\mathrm{OH})_{2}$ can also be converted to $\mathrm{FeOOH}$ in an alkaline solution.

$$
\begin{gathered}
\mathrm{Fe}_{2} \mathrm{O}_{3}+3 \mathrm{H}_{2} \mathrm{O}+2 \mathrm{e}^{-} \rightarrow 2 \mathrm{Fe}(\mathrm{OH})_{2}+2 \mathrm{OH}^{-} \\
2 \mathrm{Fe}(\mathrm{OH})_{2}+2 \mathrm{OH}^{-} \rightarrow \mathrm{FeOOH}+\mathrm{H}_{2} \mathrm{O}+\mathrm{e}^{-}
\end{gathered}
$$

Recent findings have shown that $\beta$-FeOOH nanorods can also facilitate NRR but in $0.5 \mathrm{M} \mathrm{LiClO}_{4}$ solution under atmospheric conditions with a high $\mathrm{NH}_{3}$ yield rate of $23.32 \mu \mathrm{g} \mathrm{h}^{-1} \mathrm{mg}^{-1}$ cat. and an FE of $6.7 \%$ at $-0.75 \mathrm{~V}$ (vs. RHE) and $-0.70 \mathrm{~V}$ ( $v s$. RHE), respectively. ${ }^{261}$ Similarly, our group demonstrated a higher $\mathrm{NH}_{3}$ yield rate of $27.3 \mu \mathrm{g} \mathrm{h}^{-1} \mathrm{mg}_{\text {cat. }}{ }^{-1}$ and $14.6 \% \mathrm{FE}$ at $-0.4 \mathrm{~V}$ (vs. RHE) while using $\mathrm{FeOOH}$ quantum dot decorated graphene sheets (FeOOH QDs-GSs) under ambient conditions. ${ }^{262}$ SalazarVillalpando and coworkers revealed that proton adsorption on this surface is suppressed by the presence of halide anions resulting in the boosting of catalytic activity. ${ }^{263}$ On this account, $\mathrm{Zhu}$ and co-workers assessed the catalytic characteristic of fluorine-doped $\beta$-FeOOH nanorods $(\beta-\mathrm{FeO}(\mathrm{OH}, \mathrm{F}) / \mathrm{CP})$. Under this circumstance, the presence of the $\mathrm{F}$ atom enhanced the electrocatalytic NRR performance of $\beta$-FeOOH nanorods by reducing the overpotential. Moreover, the authors concluded using DFT calculations that the lowering of the overpotential and improved activity result from the reduction in the reaction energy barrier by the $\mathrm{F}$ atom. ${ }^{264}$ Hence, this approach is highly favorable to enhance Fe-based electrocatalysts for NRR processes involving neutral solutions. Xiang and coworkers established that $\mathrm{Fe}_{2} \mathrm{O}_{3}$ catalysts can activate the NRR process in a neutral medium. ${ }^{265}$ However, it was reported that $\mathrm{Fe}_{2} \mathrm{O}_{3}$ reduced to other Fe-species such as $\mathrm{Fe}_{3} \mathrm{O}_{4}$ and $\mathrm{Fe}$ at large negative potentials. Remarkably, $\mathrm{Fe}_{3} \mathrm{O}_{4}$ possesses higher electronic conductivity than $\mathrm{Fe}_{2} \mathrm{O}_{3}$, demonstrating the potential to function as a superior catalyst system. ${ }^{266}$ Recent studies have supported this claim by presenting $\mathrm{Fe} / \mathrm{Fe}_{3} \mathrm{O}_{4}$ as a suitable NRR electrocatalyst in $0.1 \mathrm{M}$ PBS under atmospheric conditions. ${ }^{267}$ It is noteworthy that the PBS electrolyte was selected in this case due to its ability to effectively suppress the HER. ${ }^{268}$ Due to the co-occurrence of Fe and $\mathrm{Fe}_{3} \mathrm{O}_{4}$, an actual electrochemical mechanism of these $\mathrm{Fe}_{3} \mathrm{O}_{4}$ catalysts is still unclear. Subsequent studies investigating the properties of Fe-based compounds towards $\mathrm{N}_{2}$ fixation revealed that compound centers that possess high-spin polarization can better function effectively as active centers to promote nitrogen uptake and activation at low temperatures. ${ }^{269}$ Besides, studies have shown that the $\mathrm{Fe}_{3} \mathrm{O}_{4}$ nanorod is also a viable electrocatalyst for $\mathrm{NH}_{3}$ synthesis under ambient conditions. ${ }^{270}$

Owing to their strong affinity towards $\mathrm{N}$-adatoms/admolecules than $\mathrm{H}$-adatoms, $\mathrm{Ti}$ and its oxides are also highly considered as catalyst systems for electrochemical $\mathrm{NH}_{3}$ synthesis. However, when acting alone, Ti-based materials have low NRR activity owing to their poor electronic conductivity. ${ }^{271}$ To circumvent this, Ti-based catalysts are supported on conductive substrates such as RGO $\left(\mathrm{TiO}_{2}\right.$-rGO). When tested in a neutral solution $\left(0.1 \mathrm{M} \mathrm{Na}_{2} \mathrm{SO}_{4}\right)$, an ammonia yield rate of $15.13 \mu \mathrm{g} \mathrm{h}^{-1} \mathrm{mg}^{-1}$ cat. and $3.3 \% \mathrm{FE}$ at $-0.90 \mathrm{~V}$ (vs. RHE) were observed over $\mathrm{TiO}_{2}$-rGO (Fig. 6(e) and (f)). ${ }^{272}$

Moreover, C-doping has shown great potential for improving the electro-conducting state of $\mathrm{TiO}_{2}{ }^{281}$ When doped with carbon, C-doped $\mathrm{TiO}_{2}$ NPs displayed a high $\mathrm{NH}_{3}$ yield rate of $16.22 \mu \mathrm{g} \mathrm{h}^{-1} \mathrm{mg}^{-1}$ cat. and a FE of $1.84 \%$ at $-0.7 \mathrm{~V} v s$. RHE in $0.1 \mathrm{M} \mathrm{Na}_{2} \mathrm{SO}_{4}$ (Fig. 6(g) and (h)). ${ }^{273}$ Compared to the effect of C-dopant, B-dopants as an electron deficient atom could enrich the positively charged centers for $\mathrm{N}_{2}$ adsorption and activation (Fig. 6(i) and (j)). B-doped $\mathrm{TiO}_{2}$ produced $\mathrm{NH}_{3}$ at a rate of $14.4 \mu \mathrm{g} \mathrm{h}^{-1} \mathrm{mg}^{-1}$ cat. at $-0.8 \mathrm{~V}$ vs. RHE in $0.1 \mathrm{M} \mathrm{Na}_{2} \mathrm{SO}_{4},{ }^{274}$ which was slightly lower than that of C-doped $\mathrm{TiO}_{2} \mathrm{NPs}^{273}$ However, the former demonstrated a higher FE of about $3.4 \%$ when compared to the $1.84 \%$ of the latter. The variation in the activity was related to the transitioning of the semiconducting phase of $\mathrm{TiO}_{2}$ into a semi-metal state due to the appropriate B-doping resulting in the transfer of more electrons to expedite $\mathrm{N}_{2}$ activation. Also, the introduction of B-dopant resulted in the formation of O-vacant defects on the $\mathrm{TiO}_{2}$ surface which enabled the trapping of electrons at the vacant active sites for severing the $\mathrm{N} \equiv \mathrm{N}$ bond. ${ }^{274}$

In view of the presence of defects, it is conceived that the occurrence of O-vacancies of NRR electrocatalysts can result in 

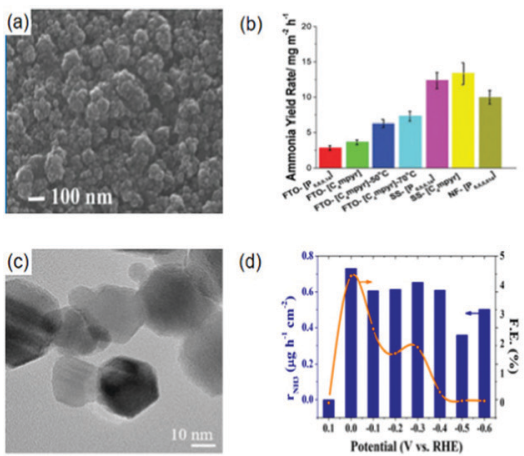

(d)
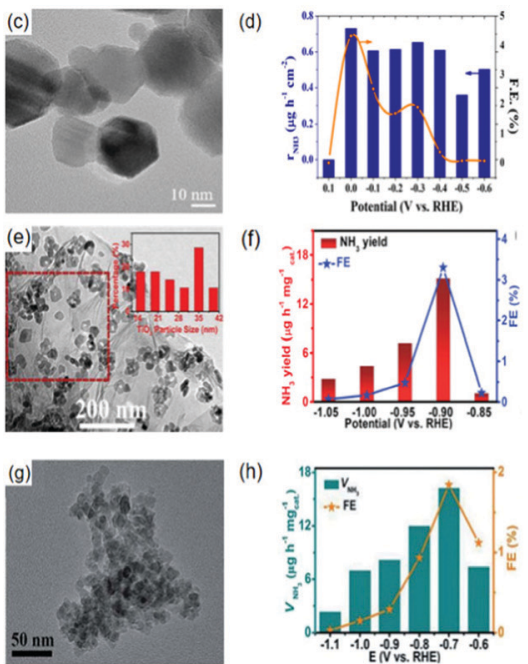

(h)

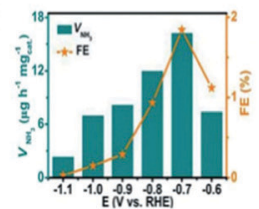

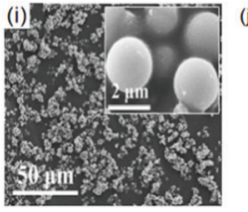
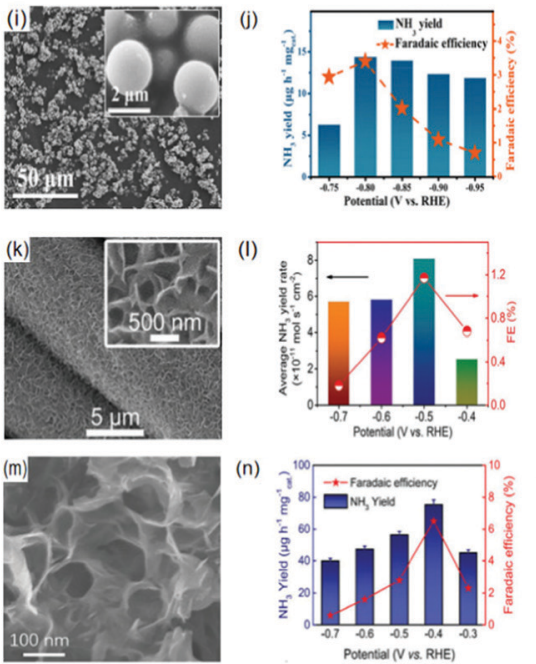

(n)
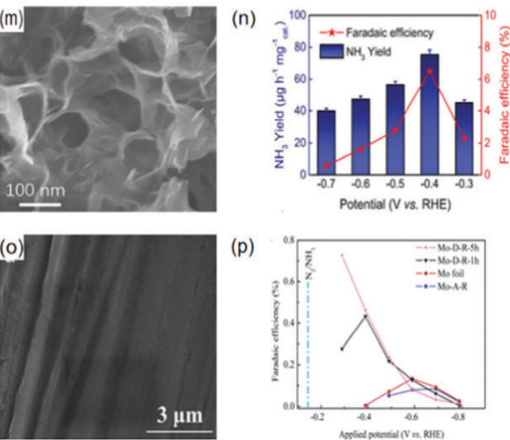
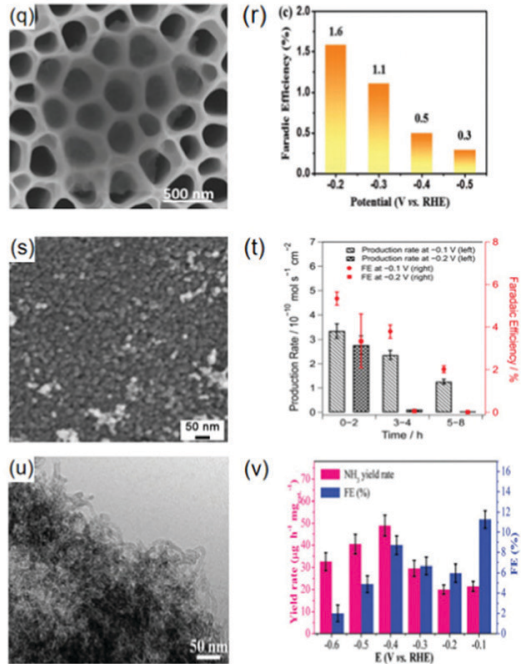

(v)
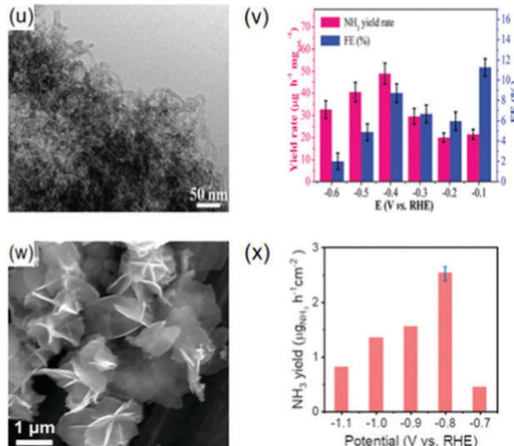

Fig. 6 (a) SEM image of the Fe-based catalyst and the corresponding (b) $\mathrm{NH}_{3}$ yield rate on different electrodes and ionic liquids at $-0.8 \mathrm{~V}$ (vs. $\mathrm{NHE}$ ). Images (a) and (b) are reproduced with permission. ${ }^{258}$ Copyright 2017, Royal Society of Chemistry. (c) TEM image of $\gamma-\mathrm{Fe}_{2} \mathrm{O}_{3} \mathrm{NPs}$, and the corresponding (d) $\mathrm{NH}_{3}$ yield rate and $\mathrm{FE}$ at different potentials in $\mathrm{N}_{2}$-saturated $0.1 \mathrm{M} \mathrm{KOH}$. Images (c) and (d) are reproduced with permission. ${ }^{255}$ Copyright 2017 , American Chemical Society. (e) TEM image of $\mathrm{TiO}_{2}-\mathrm{rGO}$ (inset: the particle size distribution of $\mathrm{TiO}_{2}$ ) and its corresponding (f) $\mathrm{NH}_{3}$ yield rate and $\mathrm{FE}$ at different potentials in $0.1 \mathrm{M} \mathrm{Na}_{2} \mathrm{SO}_{4}$. Images (e) and (f) are reproduced with permission. ${ }^{272}$ Copyright 2018, Royal Society of Chemistry. (g) TEM image of $\mathrm{C}-\mathrm{TiO}_{2} \mathrm{NP}$ and its corresponding (h) $\mathrm{NH}_{3}$ yield rate and $\mathrm{FE}$ at different potentials in $0.1 \mathrm{M} \mathrm{Na}_{2} \mathrm{SO}_{4}$. Images $(\mathrm{g})$ and (h) are reproduced with permission. ${ }^{273}$ Copyright 2019, Royal Society of Chemistry. (i) SEM image of B- $\mathrm{TiO}_{2}$ and its corresponding (j) $\mathrm{NH}_{3}$ yield rate and $\mathrm{FE}$ at different potentials in $0.1 \mathrm{M} \mathrm{Na}_{2} \mathrm{SO}_{4}$. Images (i) and (j) are reproduced with permission. ${ }^{274}$ Copyright 2019. American Chemical Society. (k) SEM image of $\mathrm{MoS}_{2} / \mathrm{CC}$ and its $\mathrm{COrresponding}$ (l) $\mathrm{NH}_{3}$ yield rate and FEs at different potentials. Images (k) and (l) are reproduced with permission. ${ }^{275}$ Copyright 2018, Wiley. (m) SEM image of Fe $\mathrm{S}_{4}$ nanosheets and their corresponding $(\mathrm{n}) \mathrm{NH}_{3}$ yield rate and FEs at different potentials. Images $(\mathrm{m})$ and $(\mathrm{n})$ are reproduced with permission. ${ }^{276}$ Copyright 2020, Wiley. (o) SEM image of the (110)-oriented Mo nanofilm and its corresponding (p) FE at different potentials. Images (o) and (p) are reproduced with permission. ${ }^{245}$ Copyright 2017, Royal Society of Chemistry. (q) SEM image of $\mathrm{Mo}_{2} \mathrm{C} / \mathrm{C}$ nanosheets and their corresponding (r) FE at different potentials. Images (q) and (r) are reproduced with permission. ${ }^{277}$ Copyright 2018, Wiley. (s) SEM image of VN nanoparticles. (t) Time-dependent production rate and faradaic efficiency at $-0.1 \mathrm{~V}$ and $-0.2 \mathrm{~V}$ for $8 \mathrm{~h}$ tests, respectively. Images (s) and (t) are reproduced with permission. ${ }^{278}$ Copyright 2018 , American Chemical Society. (u) TEM image of CoP/CNs and their corresponding (v) $\mathrm{NH}_{3}$ yield rate and FE at different potentials. Images (u) and (v) are reproduced with permission. ${ }^{279}$ Copyright 2019, Royal Society of Chemistry. (w) SEM image and (x) $\mathrm{NH}_{3}$ formation rates at different potentials of Bi NSs. Images (w) and $(x)$ are reproduced with permission. ${ }^{280}$ Copyright 2020 , Royal Society of Chemistry.

the generation of $\mathrm{H}^{+}$defects which can trap electrons for the activation of $\mathrm{N}_{2} \cdot{ }^{282}$ For instance, Zhang and co-workers highlighted the significant role of O-vacant sites in the $\mathrm{N}_{2}$-to- $\mathrm{NH}_{3}$ conversion over $\mathrm{TiO}_{2}$. Here, a yield rate of $5.62 \mu \mathrm{g} \mathrm{h} \mathrm{h}^{-1} \mathrm{~cm}^{-2}$ with $2.5 \% \mathrm{FE}$ was achieved at $-0.7 \mathrm{~V}$ vs. RHE while utilizing defective $\mathrm{TiO}_{2}$ in $0.1 \mathrm{M} \mathrm{Na}_{2} \mathrm{SO}_{4}{ }^{283}$ In other studies, the presence of $\mathrm{O}$-vacancies was credited for the increased activity over $\mathrm{TiO}_{2}$ to $7.59 \mu \mathrm{g} \mathrm{h}^{-1} \mathrm{~cm}^{-2}$ from $1.04 \mu \mathrm{g} \mathrm{h}^{-1} \mathrm{~cm}^{-2}$ for the perfect $\mathrm{TiO}_{2}$ in an acidic medium. Moreover, an increase in FE was also observed from $0.95 \%$ to $9.17 \%$ under ambient conditions. $^{284}$

Metal chalcogenides. Following the significant role of $\mathrm{S}$ and Mo in nitrogenases for $\mathrm{N}_{2}$ fixation, studies have shown that the presence of S atoms can enhance the NRR activity of Mo. ${ }^{275}$ In addition to this, the preliminary study on the electroreduction of $\mathrm{NH}_{3}$ by Furuya and Yoshiba in 1990 revealed that metal chalcogenides (comprising of sulfides and selenides) displayed best NRR activity, with ZnSe being the most suitable for the reduction of $\mathrm{N}_{2} \cdot{ }^{14}$ However, the $\mathrm{FE}$ was relatively low and was attributed to the relative competition of the HER. In this case again, suppressing the HER activity is a challenge to be confronted. Hence, the development of this set of electrocatalysts is crucial towards an effective NRR with high $\mathrm{NH}_{3}$ yield.

Recently, Sun and co-workers reported the effectiveness of $\mathrm{MoS}_{2}$ nanosheets grown on carbon cloth (Fig. 6(k)) to serve as an NRR electrocatalyst yielding $\mathrm{NH}_{3}$ at a rate of $4.945 \mu \mathrm{g} \mathrm{h}^{-1} \mathrm{~cm}^{-2}$ with $1.17 \% \mathrm{FE}$ at $-0.5 \mathrm{~V}$ (vs. RHE) in $0.1 \mathrm{M} \mathrm{Na}_{2} \mathrm{SO}_{4}$ solution (Fig. 6(l)). ${ }^{275}$ Regarding the NRR performance of $\mathrm{MoS}_{2}$, Suryanto and co-workers related the suppression of HER to the enriched $\mathrm{N}_{2}$ binding sites partly resulting from the occurrence of isolated S-vacancy defects, which served as centers for hydrogenation. ${ }^{285}$

Theoretically, Abghoui and co-workers investigated the NRR potential of several TM sulphides via a DFT computational 
study. After structural optimization, computational results revealed that $\mathrm{RuS}_{2}$ is the most active among all examined model catalysts that could catalyze the $\mathrm{N}_{2}$-to- $\mathrm{NH}_{3}$ conversion at potentials around $-0.3 \mathrm{~V}$ through the associative mechanism. NbS, CrS, TiS, and VS are also promising NRR catalyst systems with both associative and dissociative mechanisms at overpotentials ranging from 0.7 to $1.1 \mathrm{~V} .^{286}$ In addition to these sulfides, metal selenides have also demonstrated good NRR activity under ambient conditions. Recent development has fabricated selenium vacancy rich $\mathrm{ReSe}_{2}$ @carbonized bacterial cellulose as an active electrocatalyst to attain an $\mathrm{NH}_{3}$ formation rate of $28.3 \mu \mathrm{g} \mathrm{h}^{-1} \mathrm{~cm}^{-2}$ with $42.5 \% \mathrm{FE}$ at $-0.25 \mathrm{~V}$ (vs. RHE) (Fig. 6(m) and (n)). ${ }^{276}$

Metal carbides. Another suitable candidate as an efficient NRR electrocatalyst is metal carbides (also known as TM carbides, TMCs). Apparently, the adsorption and activation of $\mathrm{N}_{2}$ depend on the electronic structure of the constituting elements of the electrocatalyst. Elements with unoccupied d-orbitals are more suited to interchange electrons with $\mathrm{N}$-admolecules, which will enable $\mathrm{N}_{2}$ activation. Besides the noble metals that offer these rare abilities, TMCs are predicted to display a similar concept due to the presence of unoccupied d-orbitals. ${ }^{287}$ Coupled with this, the hybridized orbitals between the TM (sp-orbital) and the carbide (s-orbital) will facilitate more back-donation to the $\pi$ orbitals of $N_{2}$, thereby enhancing $\mathrm{N}_{2}$ fixation.

As mentioned earlier, a well-known metal carbide with high catalytic activity is molybdenum carbide $\left(\mathrm{Mo}_{2} \mathrm{C}\right)$ which has displayed strong affinity towards electron-rich compounds and activation of the HER. ${ }^{288,289}$ When compared to the conventional Mo electrocatalysts (Fig. 6(o) and (p)), ${ }^{245} \mathrm{Mo}_{2} \mathrm{C}$ nanodots displayed a significant improvement in $\mathrm{NH}_{3}$ yield and efficiency (Fig. 6(q) and (r)). In addition to the inactivation of spilled over $\mathrm{H}$-adatoms by the inlaid structure, other factors that contributed to the improved ammonia yield include enhanced $\mathrm{N}_{2}$ adsorption and activation on the enriched sizepromoted active sites and the reduction of $\mathrm{H}$-coverage on the catalyst surface. Despite the good NRR performance, the occurrence of a high HER activity was observed. ${ }^{277}$ To overcome this limitation, it was suggested that inducing C-vacancy defects is likely to fortify the metal-C ratio in order to retard the accumulation of $\mathrm{H}$-adatoms and thereby evolution of $\mathrm{H}_{2}{ }^{290}$

Recently, a new family of two-dimensional (2D) TM carbides and carbonitrides, also known as MXenes $\left(\mathrm{TM}_{n+1} \mathrm{X}_{n}(n=1-3\right.$, and $\mathrm{X}=\mathrm{C}$ and/or $\mathrm{N}$ )), have presented good catalytic activity towards the electrosynthesis of $\mathrm{NH}_{3} \cdot{ }^{262,291}$ These compounds have specific structural characteristics given that their lattice TMs are exposed on both sides of the 2D layers and mostly terminated by $\mathrm{F}, \mathrm{O}$ and/or $\mathrm{OH}$ groups with the general formula $\mathrm{TM}_{n+1} \mathrm{~N}_{m} \mathrm{X}_{n}\left(\mathrm{~N}=\mathrm{F}, \mathrm{OH}\right.$, and/or O). ${ }^{292}$ Given their nascent discovery, thorough investigative studies on their mechanism are unknown. However, it is alleged that $\mathrm{O}$ - and $\mathrm{OH}$-terminated MXenes are the most catalytically viable given their stability and remarkable charge distribution. In addition, theoretical evidence has revealed that the O-terminated MXenes are active centers for HER which can be exploited to retard the HER process. $^{291}$

Based on quantum theory, the synergistic coupling effect of TMs and the integrated C-atom resulting in the unique hybridization of their orbitals enables the TMCs to behave as catalysts with electron-enriched characteristics. ${ }^{287}$ Specifically, there is a shift in the d-band of the TM upon the integration of the TM and $\mathrm{C}$ atoms, which will facilitate its hybridization with the $\mathrm{C}$ s-orbital resulting in electron-enriched orbitals that can offer more electrons to severe the $\pi$-orbitals of $\mathrm{N}_{2} \cdot{ }^{277}$ Theoretical studies to this effect have revealed the resultant low activation energy ( 0.32 and $0.39 \mathrm{eV} v s$. SHE) for the $\mathrm{N}_{2}$-to- $\mathrm{NH}_{3}$ conversion on $\mathrm{V}_{3} \mathrm{C}_{2}$ and $\mathrm{Nb}_{3} \mathrm{C}_{2}$, respectively. ${ }^{293}$

To gain insights into the NRR mechanistic pathway on TMCs, Shao and co-workers investigated the $\mathrm{N}_{2}$-fixation mechanism on MXenes. It was reported that the overall NRR energy is decided by electron transfer between the TMC and $\mathrm{N}_{2}$. Specifically, the donation and reception of more electrons from the TMCs are likely to indicate an exothermic and endothermic reaction, respectively. Hence, for an effective $\mathrm{N}_{2}$-to- $\mathrm{NH}_{3}$ conversion, more exothermic reaction, an extended $\mathrm{N}-\mathrm{N}$ bond and substantial charge transfer are required. Based on this, mechanism-guided prediction shows that $\mathrm{Mo}_{2} \mathrm{C}$ and $\mathrm{W}_{2} \mathrm{C}$ are more suitable for electrosynthesis of $\mathrm{NH}_{3}{ }^{222}$ Subsequent experimental studies validated this claim by demonstrating that $\mathrm{Mo}_{2} \mathrm{C}$ nanorods yielded $\mathrm{NH}_{3}$ at a remarkable rate of $95.1 \mu \mathrm{g} \mathrm{h}^{-1} \mathrm{mg}^{-1}$ cat and $8.13 \% \mathrm{FE}$ at $-0.3 \mathrm{~V}$ (vs. RHE) in $0.1 \mathrm{M}$ HCl. ${ }^{294}$ Another reported TMC for $\mathrm{NH}_{3}$ synthesis is the $\mathrm{F}$ - and OH-terminated $\mathrm{Ti}_{3} \mathrm{C}_{2} \mathrm{~N}_{x}(\mathrm{~N}=\mathrm{F}, \mathrm{OH})$ nanosheets which displayed a yield rate of $20.4 \mu \mathrm{g} \mathrm{h}^{-1} \mathrm{mg}^{-1}$ cat. and $9.3 \% \mathrm{FE}$ at $-0.4 \mathrm{~V}$ (vs. RHE). ${ }^{270}$

Metal nitrides. Similar to TMCs, metal nitrides (also known as TMNs) are fabricated by integrating the $\mathrm{N}$-atom into the skeletal structure of the TM resulting in the d-band contraction with an electronic structure similar to that of noble metals. ${ }^{295}$ Owing to the large distinction in electronegativity between the $\mathrm{N}$ atom and the TMs, TMNs possess enriched acid-base active centers resulting from the enhanced distribution of electron charges. The availability of the distributed active centers makes TMNs suitable candidates for $\mathrm{NH}_{3}$ electrosynthesis.

Like the TMs, TMNs can also enable the synthesis of $\mathrm{NH}_{3}$ by the direct reduction of the incorporated $\mathrm{N}$ atom, thereby creating a N-vacancy on the surface of the TMN catalyst which can also be repaired via $\mathrm{N}_{2}$ adsorption. ${ }^{215}$ Nonetheless, this pathway may be hindered by the competitive adsorption of other species other than $\mathrm{N}_{2}$ and consequently preventing the regeneration of the catalyst. On this account, two major conditions are necessary for the design of these catalysts. These conditions should include the repair and regeneration of the vacancies and a minimal overpotential.

In recent studies, theoretical evidence has presented the preferential repair of the vacant sites by $\mathrm{N}$-atoms rather than $\mathrm{H}^{+}, \mathrm{O}^{2-}$,or $\mathrm{OH}^{-}$species owing to the strong energetics between these sites towards $\mathrm{N}$-adatoms. ${ }^{296}$ The $\mathrm{N}$-adatom adsorption energies vary with the different facets of the TMNs. Upon optimization, 
calculation results revealed that the rock-salt (RS) structures with (100) facets of the nitrides of $\mathrm{V}$ and $\mathrm{Zr}$ are the most favorable catalyst systems for high yield of $\mathrm{N}_{2}$ fixation at low overpotentials, while the nitrides of $\mathrm{V}$ and $\mathrm{Cr}$ are more suited for high efficiencies owing to low $\mathrm{H}_{2}$ generation. ${ }^{205}$ Besides, the (111) facet of the RS for $\mathrm{MnN}, \mathrm{VN}$ and $\mathrm{CrN}$ also demonstrated a low overpotential towards $\mathrm{N}_{2}$-to- $\mathrm{NH}_{3}$ conversion. Nevertheless, $\mathrm{MnN}$ is preferentially attacked by other species other than $\mathrm{N}_{2}$ and thereby its performance as a NRR electrocatalyst is hindered. ${ }^{295}$

Founded on these theoretical calculations, several experimental studies have been conducted on VN as a suitable NRR electrocatalyst with more active centers for a high yield and conversion efficiency of $\mathrm{N}_{2}$-to- $\mathrm{NH}_{3}$. These studies include the evaluation of the VN nanosheet array on Ti mesh, ${ }^{297} \mathrm{VN}$ NPS (Fig. 6(s) and (t) $)^{278}$ and VN nanowire array (on CC). ${ }^{298}$ Using the trackable ${ }^{15} \mathrm{~N}_{2}$ species as the feed gas, it was evident that the NRR route on VN followed the Mars-van Krevelen (MvK) mechanism with the lattice $\mathrm{N}$-atom partaking in the formation of ${ }^{14} \mathrm{NH}_{3}$ and ${ }^{15} \mathrm{NH}_{3}$, and subsequent healing of the vacant site thereafter created. ${ }^{278}$

Metal phosphides. Among the myriads of catalyst systems for $\mathrm{N}_{2}$-fixation under ambient conditions, transition metal phosphides (TMPs) have shown good potential in this regard given their high catalytic efficacy, earth-abundance and low-cost. In addition to this, it is stipulated that the positively charged metal sites of the TMPs serve as the active centers for $\mathrm{N}_{2}$ adsorption and activation, while the adjacent negatively charged P-motifs are sites for proton anchoring via H-bonding. This electronic modulating effect of phosphorus has attracted research attention towards its adoption to boost the intrinsic characteristics of NRR electrocatalysts.

Experimentally, Zhang and co-workers investigated the potential of CoP NPs synthesized via the pyrolysis-phosphorization method as NRR electrocatalysts under ambient conditions. Here, the authors demonstrated that the as-synthesized CoP/CNs yielded $\mathrm{NH}_{3}$ at a rate of $48.9 \mu \mathrm{g} \mathrm{h}^{-1} \mathrm{mg}^{-1}$ cat and $8.7 \% \mathrm{FE}$ at $-0.4 \mathrm{~V}$ (vs. RHE) in $0.1 \mathrm{M} \mathrm{Na}_{2} \mathrm{SO}_{4}$ with an associative distal mechanism (Fig. 6(u) and (v)). ${ }^{279}$ Recently, Zhang and co-workers also revealed the significant role of support effects in the modulation of surficial electronic characteristics of $\mathrm{Ni}_{2} \mathrm{P}$ NPs. ${ }^{280}$ In this study, $\mathrm{Ni}_{2} \mathrm{P}$ NPs supported on N,P co-doped $\mathrm{CNs}\left(\mathrm{Ni}_{2} \mathrm{P} / \mathrm{N}, \mathrm{P}-\mathrm{C}\right)$ were tested in alkaline, acidic and neutral solutions under ambient conditions. The $\mathrm{Ni}_{2} \mathrm{P} / \mathrm{N}, \mathrm{P}-\mathrm{C}$ catalyst displayed an excellent catalytic activity in all electrolytes with the highest performance witnessed in $0.1 \mathrm{M} \mathrm{KOH}$ with a yield rate of $90.1 \mu \mathrm{g} \mathrm{h}^{-1} \mathrm{mg}^{-1}$ and $19.82 \% \mathrm{FE}$ at $-0.2 \mathrm{~V}$ (vs. RHE). In $0.1 \mathrm{M} \mathrm{HCl}$, a yield rate of $34.4 \mu \mathrm{g} \mathrm{h}^{-1} \mathrm{mg}^{-1}$ and $17.21 \% \mathrm{FE}$ were witnessed (Fig. 6(w) and (x)). ${ }^{280}$ The high activity of $\mathrm{Ni}_{2} \mathrm{P} / \mathrm{N}, \mathrm{P}-\mathrm{C}$ was attributed to the appropriate modulating effect of the N,P-C substrate to trap and distribute electrons. Following the establishment of the unusual role of phosphorus in modulating the NRR activity of catalyst systems and the importance of support effects, we demonstrated the high NRR performance of the $\mathrm{FeP}_{2}$ NP-RGO hybrid. When tested in $0.5 \mathrm{M} \mathrm{LiClO}_{4}$, an $\mathrm{NH}_{3}$ yield rate of $35.26 \mu \mathrm{g} \mathrm{h}^{-1} \mathrm{mg}^{-1}$ cat. and a high $\mathrm{FE}$ of $21.99 \%$ at $-0.4 \mathrm{~V}$ (vs. RHE) were witnessed. ${ }^{299}$ Theoretical evidence shows that the $\mathrm{FeP}_{2}$ offers enriched active sites, higher $\mathrm{N}_{2}$ adsorption energy and a retarding effect for the HER than FeP.

3.2.3. Metal-free electrocatalysts. As mentioned earlier, TMs are suitable catalytic candidates for electrochemical NRR due to their intrinsic electronic structure with electron interchangeability between the $\sigma$ and $\pi$ orbitals of the $\mathrm{N}_{2}$ molecule. Despite this, most TMs are incompetent to strongly bond with $\mathrm{N}_{2}$, and hence cannot aid towards the desirable level of $\mathrm{N}_{2}$ activation. Moreover, it is argued that the electrons in the d-orbital assist the interaction with $\mathrm{H}$ which promotes the HER. From an environmental standpoint, the probable release of TMs to the environment during the catalytic process partly dissuades the potential application of this set of catalysts. Under these circumstances, metal-free materials are highly attractive as electrochemical catalysts for $\mathrm{N}_{2}$-to- $\mathrm{NH}_{3}$ conversion. ${ }^{300}$ Relative to TMs, metal-free electrocatalysts are enriched with valence electrons and show weak $\mathrm{H}_{2}$ adsorption capacity, which render them suitable for $\mathrm{N}_{2}$ fixation. ${ }^{161}$

Carbon-based electrocatalysts. Carbon-based electrocatalysts are one of the favorable alternatives for metal-based NRR electrocatalysts owing to their low HER selectivity, ${ }^{301,302}$ unfavorable $\mathrm{H}_{2}$ formation at the $\mathrm{C}$ edge site ${ }^{303}$ and high electrical conductivity and electrochemical stability. ${ }^{300}$ Also, these unique electrocatalysts easily offer to back-donate their copious $\pi$ electrons to the $\pi$ orbitals of $\mathrm{N}_{2}$ leading to the activation of $\mathrm{N}$-admolecules. Nonetheless, pristine carbon-based materials still have a low NRR catalytic activity owing to the inert nature of the $\pi$ electrons. To circumvent these shortcomings, approaches to modulate the NRR activity of carbon-based electrocatalysts could include defect introduction $^{304}$ and heteroatom doping. ${ }^{305,306}$ Numerous studies have revealed that the activation of $\mathrm{N}$-admolecules on a defect-rich carbon structure is easily enabled on the defective sites. To promote these defective sites on the surface of carbon-based materials, N, B, O and S-dopants are the most commonly used dopants. However, a recent study revealed a remarkably high NRR activity $\left(\mathrm{NH}_{3}\right.$ yield rate $=32.33 \mu \mathrm{g} \mathrm{h}^{-1} \mathrm{mg}^{-1}$ cat. and $\mathrm{FE}$ of $20.82 \%$ at $0.65 \mathrm{~V}$ vs. RHE) on P-doped graphene in $0.5 \mathrm{M} \mathrm{LiClO}_{4}$ under ambient conditions (Fig. 7(a) and (b)). It was revealed that the P-dopant fostered graphene re-stacking which created more defect sites in the structure. ${ }^{306}$

$N$-Dopant. To activate the $\pi$ electrons of carbon-based electrocatalysts, the lone-pair electrons of the $\mathrm{N}$-atom readily conjugate with $\pi$ electrons, thereby making it an effective approach to enhance the NRR activity on carbon-based materials. ${ }^{307}$ In other words, $\mathrm{N}$-doped carbon materials could generate additional Lewis pairs that can readily activate $\mathrm{N}_{2}$ and $\mathrm{H}_{2}$ molecules. ${ }^{308}$ Coupled with this, $\mathrm{N}$-doped carbon materials are easily polarizable such that they promote adsorption of $\mathrm{N}_{2}$ and electron/mass transfer. ${ }^{186}$

Recently, experimental studies have involved incorporating $\mathrm{N}$-dopant into C-based structures that exhibit remarkable NRR activity. Song and co-workers demonstrated an $\mathrm{NH}_{3}$ yield rate $97.18 \pm 7.13 \mathrm{mg} \mathrm{h}^{-1} \mathrm{~cm}^{-2}$ with $11.56 \pm 0.85 \% \mathrm{FE}$ at $-1.19 \mathrm{~V}$ 


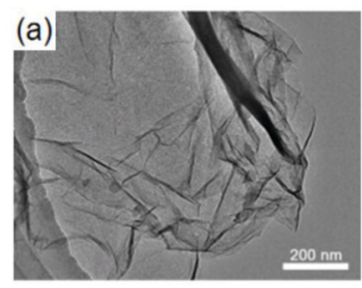

(c)
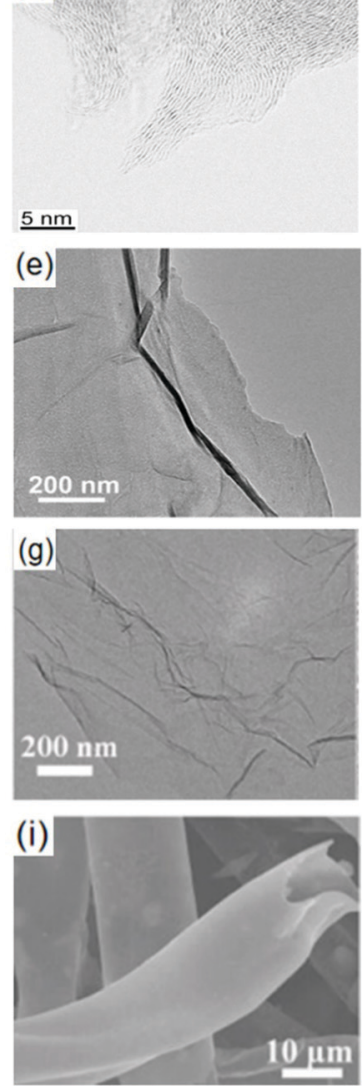

(b)

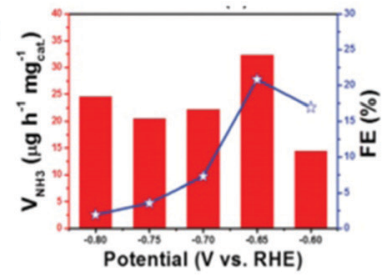

(d)

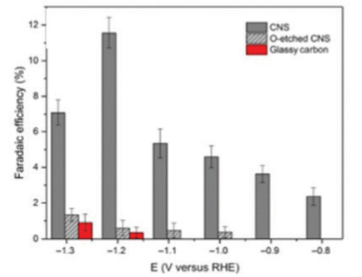

(f)

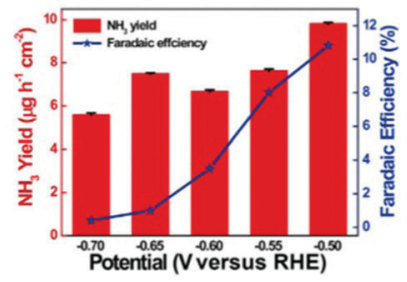

(h)

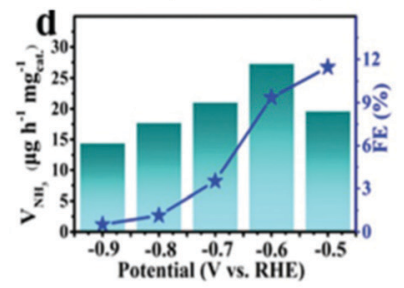

(j)

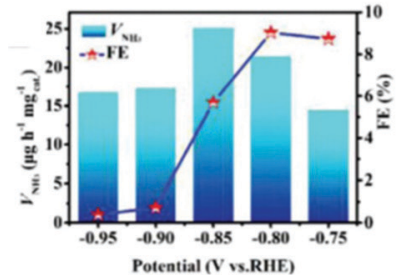

Fig. 7 (a) TEM image of P-doped graphene and its corresponding (b) $\mathrm{NH}_{3}$ yield rate and FE at different potentials. Panels (a) and (b) are reproduced with permission. ${ }^{306}$ Copyright 2020, Royal Society of Chemistry. (c) Aberration-corrected STEM image of $\mathrm{N}$-doped carbon nanospikes and their corresponding (d) FE at different potentials. Panels (c) and (d) are reproduced with permission. ${ }^{301}$ Copyright 2018, American Association for the Advancement of Science. (e) TEM image of B-doped graphene and its corresponding (f) $\mathrm{NH}_{3}$ yield rate and FE at different potentials. Panels (e) and (f) are reproduced with permission. ${ }^{316}$ Copyright 2018, Elsevier. (g) TEM image of S-doped graphene and its corresponding (h) $\mathrm{NH}_{3}$ yield rate and FE at different potentials. Panels $(\mathrm{g})$ and $(\mathrm{h})$ are reproduced with permission. ${ }^{323}$ Copyright 2019, Royal Society of Chemistry. (i) TEM image of O-doped hollow carbon microtubes and their corresponding (j) $\mathrm{NH}_{3}$ yield rate and FE at different potentials. Panels (i) and (j) are reproduced with permission. ${ }^{324}$ Copyright 2019, Royal Society of Chemistry.

(vs. RHE) over N-doped carbon nanospikes in $0.25 \mathrm{M} \mathrm{LiClO}_{4}$ (Fig. 7(c) and (d)). ${ }^{301}$ The sharp spike structure of the electrocatalyst provided a dense distribution of electrons at its tips, which promoted the dissolution of $\mathrm{N}_{2}$.

Liu and co-workers further revealed that the NRR performance of the $\mathrm{N}$-doped carbon material can be regulated by adjusting the pyridinic and pyrrolic $\mathrm{N}$ content in $\mathrm{N}$-doped porous carbon (NPC). ${ }^{186}$ Most importantly, the pyridinic $\mathrm{N}$ atom in NPC partakes in the formation of $\mathrm{NH}_{3}$ resulting in the generation of $\mathrm{N}$-vacancies that can serve as active centers for further activation of $\mathrm{N}$-admolecules. ${ }^{309}$

However, this is most effective in alkaline solutions as NPC undergoes severe HER in acidic electrolytes. ${ }^{310}$ Other reported $\mathrm{N}$-doped carbon-based NRR catalysts include polymeric carbon nitride (PCN) with an enhanced spatial electron transfer due to the induced N-vacancies. ${ }^{311,312}$

B-Dopant. Similar to N-dopants, B-dopants have also shown great capacity to activate the $\pi$ electrons of carbon-based electrocatalysts. Moreover, boron is a notable single atom catalyst for the electrochemical $\mathrm{N}_{2}$-to- $\mathrm{NH}_{3}$ transformation. In addition to their successful NRR performance, ${ }^{313,314}$ B-dopants like $\mathrm{N}$-dopants have equally attracted great attention as a means to modulate the NRR activity of carbon-based electrocatalysts. $^{315,316}$

Boron is an electron deficient atom with four valence electrons in the sp-orbitals, which bonds uniquely with the electronic structure of $\mathrm{C}^{317}$ The hybridized electronic structure between these two atoms results in the generation of unoccupied orbitals that can accept lone-pair electrons from $\mathrm{N}_{2}$. Simultaneously, the occupied 2p-orbitals can back donate electrons to the $\pi$ orbitals of $\mathrm{N}$-admolecules. Above all, Batoms can retard the HER by prohibiting the binding of Lewis acids in an acidic medium. ${ }^{316,318,319}$

Despite the good electronic structure, this set of electrocatalysts still suffer from high HER activity and instability of adsorbed $\mathrm{N}_{2}$, therefore requiring appropriate schemes to strengthen it. ${ }^{315}$ In this regard, optimizing the content of B-dopant has been shown to mitigate this shortcoming. For example, B-doped graphene exhibited an optimum NRR activity at a B-dopant content of $6.2 \%$ with a yield rate of $9.8 \mu \mathrm{g} \mathrm{h}^{-1} \mathrm{~cm}^{-2}$ and a high $\mathrm{FE}$ of $10.8 \%$ at $-0.5 \mathrm{~V}$ (vs. RHE) in $0.05 \mathrm{M} \mathrm{H}_{2} \mathrm{SO}_{4}$ solution (Fig. 7(e) and (f)). ${ }^{316}$ As mentioned earlier, $\mathrm{S}$ plays a significant role in the biological synthesis of $\mathrm{NH}_{3}$ by means of nitrogenase enzymes. ${ }^{320}$ Nonetheless, the low electrical conductivity of $S^{321}$ impedes its application in the electrocatalytic NRR. To circumvent this, the adoption of conductive supports such as RGO has been proposed as a suitable mechanism for boosting the electrical conductivity of S. For example, the S-doped dotsgraphene nanohybrid demonstrated a good $\mathrm{NH}_{3}$ synthesis rate of $28.56 \mu \mathrm{g} \mathrm{h}^{-1} \mathrm{mg}^{-1}$ cat. and $7.07 \% \mathrm{FE}$ at $-0.85 \mathrm{~V}$ (vs. RHE) in $0.5 \mathrm{M} \mathrm{LiClO}_{4}{ }^{322}$ Similar to other dopants, the $\mathrm{S}$ atom also possesses a modulating effect to tune the NRR activity for carbonbased materials. A typical example of this effect is portrayed in the NRR performance of graphene in $0.1 \mathrm{M} \mathrm{HCl}$ under ambient conditions. Prior to S-doping, the NRR activity on graphene resulted in an $\mathrm{NH}_{3}$ yield rate of $6.25 \mu \mathrm{g} \mathrm{h}^{-1} \mathrm{mg}^{-1}$ cat. and a low $\mathrm{FE}$ of $0.52 \%$. However, after S-doping, a significant boost in the activity was observed with an $\mathrm{NH}_{3}$ yield rate of $27.3 \mu \mathrm{g} \mathrm{h}^{-1} \mathrm{mg}^{-1}$ cat. and a high FE of $11.5 \%$ under similar conditions (Fig. $7(\mathrm{~g})$ and $(\mathrm{h}))^{323}$

O-Dopant. Similar to $\mathrm{S}$ atoms, $\mathrm{O}$ atoms possess the same electronic structure and have displayed good potential to 
improve the electrocatalytic activity of carbon-based materials. In the same manner as $\mathrm{S}$ atoms, O-doping can trigger the activity of the inert $\pi$ electrons of the carbon-based materials via $\mathrm{O}-\mathrm{C}$ interactions. An example of this is demonstrated over an amorphous O-doped carbon nanosheet (CN) with a high $\mathrm{NH}_{3}$ yield rate of $20.15 \mu \mathrm{g} \mathrm{h}^{-1} \mathrm{mg}^{-1}$ cat. and a $\mathrm{FE}$ of $4.97 \%$ at $-0.6 \mathrm{~V}$ (vs. RHE) in $0.1 \mathrm{M} \mathrm{HCl}^{300}$ Likewise, O-doped hollow carbon microtubes revealed a superior NRR performance with a yield of $25.12 \mu \mathrm{g} \mathrm{h}^{-1} \mathrm{mg}^{-1}$ cat. and 9.1\% FE at $-0.85 \mathrm{~V}$ (vs. RHE) and $-0.80 \mathrm{~V}$ (vs. RHE) in $0.1 \mathrm{M} \mathrm{HCl}$, respectively (Fig. 7(i) and (j)). ${ }^{324}$

Elementals and their derivatives. TMs are highly recommended for NRR electrocatalysis but their selectivity towards ammonia synthesis is low. Other alternatives to replace this set of catalyst systems are the elementals and their compounds owing to their low-coordinated atoms. ${ }^{277}$ Moreover, elemental catalysts are reported to significantly retard the HER based on the structural effect as the H-motifs are preferentially adsorbed on specific sites on the catalyst. ${ }^{325}$ Supported by this ensemble effect, the suppression of the HER can be controlled.

A notable elemental catalyst for electrochemical reduction of $\mathrm{N}_{2}$ is boron (B) and its compounds particularly boron nitride (BN), which have been proven to offer good NRR activity as electrocatalysts, ${ }^{313,314}$ despite theoretical studies suggesting that $\mathrm{B}-\mathrm{N}$ pairs in h-BN are inactive towards $\mathrm{N}_{2}$-to- $\mathrm{NH}_{3}$ conversion. ${ }^{326}$ For instance, Zhang and co-workers experimentally demonstrated the electrocatalytic efficacy of the $\mathrm{B}$ nanosheet to attain an $\mathrm{NH}_{3}$ yield rate of $13.22 \mu \mathrm{g} \mathrm{h}^{-1} \mathrm{mg}_{\text {cat }}{ }^{-1}$ and $4.04 \% \mathrm{FE}$ in $0.1 \mathrm{M} \mathrm{Na}_{2} \mathrm{SO}_{4} \cdot{ }^{327}$ Also, at high $\mathrm{B}$ concentration, the boron carbide $\left(\mathrm{B}_{4} \mathrm{C}\right)$ nanosheet attained a high $\mathrm{NH}_{3}$ yield rate of $26.57 \mu \mathrm{g} \mathrm{h}^{-1} \mathrm{~cm}^{-2}$ and a high $\mathrm{FE}$ of $15.95 \%$ at $-0.75 \mathrm{~V}$ (vs. RHE). Fortunately, the catalyst displayed remarkable stability and selectivity towards $\mathrm{NH}_{3}$ formation. ${ }^{328}$

Similarly, theoretical evidence has demonstrated the possibility of a monolayer phosphorus (P) catalyst system to catalyze $\mathrm{N}_{2}$-to- $\mathrm{NH}_{3}$ conversion. ${ }^{329}$ From an experimental perspective, few-layered black $\mathrm{P}$ (BP) nanosheets were used to produce $\mathrm{NH}_{3}$ with a high yield rate of $31.37 \mu \mathrm{g} \mathrm{h}^{-1} \mathrm{mg}^{-1}$ cat. and $5.07 \% \mathrm{FE}$ at $-0.7 \mathrm{~V}$ (vs. RHE) and $-0.6 \mathrm{~V}$ (vs. RHE), respectively. In addition, the authors also revealed that the active sites for the adsorption and activation of $\mathrm{N}_{2}$ were more favorable on the zigzag and diff-zigzag edges of the BP nanosheets. On these nanosheets, computational analysis indicated that only the edges of the catalyst structure could facilitate electron donation during the NRR, which limited the performance of the catalyst. $^{330}$ To circumvent this, anchoring a single-atom Fe on the monolayer $\mathrm{P}$ was shown to vary the charge distribution and promote electron interchangeability at the edge of the catalyst system. $^{329}$

In summary, advancements in the catalyst systems to enable efficient electrochemical $\mathrm{N}_{2}$-to- $\mathrm{NH}_{3}$ conversion under ambient conditions have been substantial. An ideal catalyst system should facilitate the adsorption and activation of $\mathrm{N}_{2}$ in order to promote the NRR kinetics. Where necessary, tailoring the catalysts' electronic structure (by defect engineering, heteroatom doping, surface functionalization and interface engineering) can enhance their intrinsic NRR characteristics. More specifically, enriching the NRR active sites/centers (size and shape modification, utilizing supports with high surface area and conductivity, and anchoring single-atoms on the catalyst system) while suppressing that of the HER is the most direct means of enhancing the $\mathrm{N}_{2}$ reduction activity. ${ }^{331}$ Generally, the HER has a lower overpotential enabling it to be preferentially selected over $\mathrm{NH}_{3}$ formation; hence, the need for HER-retarding strategies such as the use of functional composite catalysts is recommended. ${ }^{332,333}$ In addition, the electrolyte selection, stability improvement and cost-competitiveness are still the important research aspects deserving attention in order to develop competitive NRR technologies towards practicality. For a brief overview, Table 2 summarizes the recent NRR electrocatalysts and their catalytic activity, providing insights into the chemical understanding of efficient electrocatalysts for NRR.

\section{Valuable fuels and chemicals via $\mathrm{CO}_{2}$ reduction reaction}

Conversion of $\mathrm{CO}_{2}$ as part of carbon capture and utilization (CCU) technologies has received increased interest in the past couple of decades, especially in view of favourable prospects related to positive impact on the global climate change and renewable electricity production. ${ }^{334,335}$ Research in this field has mainly focused on fundamental and mechanistic conversion of $\mathrm{CO}_{2}$ to valuable fuels and chemicals through a variety of technologies including biochemical, ${ }^{336}$ thermochemical, ${ }^{337}$ photochemical $^{338}$ and electrochemical ${ }^{339}$ reduction of $\mathrm{CO}_{2}$. Among these technologies, electrocatalytic $\mathrm{CO}_{2}$ reduction reaction (CRR) has attracted greater attention by virtue of its mild operating conditions and great potential to scale up. ${ }^{340-343}$ Although significant progress is experienced in this field, there are still major challenges, which hinder the understanding of CRR. ${ }^{344}$

Pioneering studies on CRR involving different metals was initiated by Hori and co-workers more than thirty years ago. ${ }^{11,345}$ However, large-scale implementation of CRR technology is still at its infant stage because, in contrast to fundamental CRR studies, the research to understand CRR from an industrial perspective and efforts to develop an industrial/ commercial $\mathrm{CO}_{2}$ electrolyzer are scarce. Due to the commercial limitations, CRR still suffers from lack of mechanistic understanding of the kinetics and thermodynamic challenges. Specifically, the $\mathrm{C}=\mathrm{O}$ double bond of the $\mathrm{CO}_{2}$ molecule possesses a high bonding energy $\left(750 \mathrm{~kJ} \mathrm{~mol}^{-1}\right)$ when compared to the binding energies of the $\mathrm{C}-\mathrm{C}$ bond $\left(336 \mathrm{~kJ} \mathrm{~mol}^{-1}\right), \mathrm{C}-\mathrm{H}$ bond $\left(411 \mathrm{~kJ} \mathrm{~mol}^{-1}\right)$ and $\mathrm{C}-\mathrm{O}$ bond $\left(327 \mathrm{~kJ} \mathrm{~mol}^{-1}\right)$ of conventional hydrocarbons. Hence, in the absence of an external support, it is energetically unfavourable to dissociate $\mathrm{CO}_{2}$ to generate organic compounds. ${ }^{346}$ It is therefore necessary to utilize catalyst systems to lower the energy barrier, stabilize major intermediates and facilitate reaction kinetics.

Moreover, based on the utilized catalyst system and operating condition, a wide range of reduced products can be generated 
from the CRR including carbon monoxide (CO), formate/formic acids $\left(\mathrm{HCOO}^{-} / \mathrm{HCOOH}\right)$, methane $\left(\mathrm{CH}_{4}\right)$, methanol $\left(\mathrm{CH}_{3} \mathrm{OH}\right)$, ethane $\left(\mathrm{C}_{2} \mathrm{H}_{4}\right)$, ethanol $\left(\mathrm{C}_{2} \mathrm{H}_{5} \mathrm{OH}\right)$ and so on. Principally, this is established by the reaction mechanism at a given condition and most importantly, the working electrocatalyst. ${ }^{347,348}$ In essence, it is a considerable challenge to mechanistically tune the reaction to enhance a particular product selectivity. ${ }^{349}$ Furthermore, this shortcoming is compounded by the kinetically competitive HER, as was also notable in the NRR and water splitting process. As an outcome, the rational design and development of catalyst systems for the electroreduction of $\mathrm{CO}_{2}$ are highly necessary.

\subsection{Electrochemistry of $\mathrm{CO}_{2}$ reduction reaction (CRR)}

4.1.1. Fundamentals of CRR. CRR is a multi-step transformation process consisting of two-, four-, six-, or eight-electron reaction pathways (Fig. $8(\mathrm{a}))^{350}$ to primarily synthesize formate $\left(\mathrm{HCOO}^{-}\right)$or formic acid $(\mathrm{HCOOH})($ Fig. $8(\mathrm{~b}))$, carbon monoxide (CO) (Fig. 8(c)), methane $\left(\mathrm{CH}_{4}\right)$, ethylene $\left(\mathrm{C}_{2} \mathrm{H}_{4}\right)$, ethanol $\left(\mathrm{C}_{2} \mathrm{H}_{5} \mathrm{OH}\right)$ (Fig. 8(d)), etc. ${ }^{341}$ The reaction kinetics is governed by the electrocatalyst, operating condition (pressure, temperature and $\mathrm{pH}$ ) and electrolytic solution, irrespective of the thermodynamics involved. Primarily, the operating conditions influence the kinetics by altering the $\mathrm{CO}_{2}$ solubility in the electrolytic solution, which is mainly favoured at high $\mathrm{CO}_{2}$ partial pressure and low temperatures. In view of all these, the CRR occurs at the interface of the electrocatalyst-containing electrode and the $\mathrm{CO}_{2}$-saturated aqueous solution. The electrocatalyst, preferably constituted of earth-abundant materials, and the electrolytic solution play an essential role in establishing the CRR mechanism and kinetics. Thus far, several research studies are focused on developing high-performance and less expensive electrocatalysts, particularly heterogeneous electrocatalysts, to optimize the CRR efficiency based on the reaction mechanism and kinetics. ${ }^{361,362}$

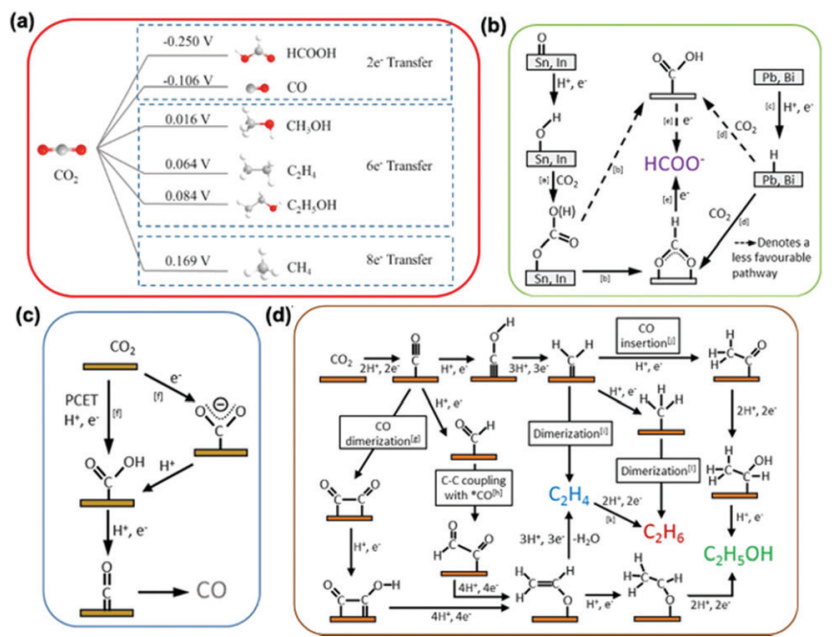

Fig. 8 (a) $\mathrm{CO}_{2}$ reduction processes and the corresponding standard redox potentials, $E^{0}$ (vs. SHE, V) for aqueous solutions. Reproduced with permission. ${ }^{350}$ Copyright 2014, Elsevier. CRR mechanisms to generate (b) formate, (c) $\mathrm{CO}$, and (d) $\mathrm{C}_{2} \mathrm{H}_{4}, \mathrm{C}_{2} \mathrm{H}_{6}$ and $\mathrm{C}_{2} \mathrm{H}_{5} \mathrm{OH}$, initiated from $\mathrm{CO}$ adsorption. Reproduced with permission. ${ }^{341}$ Copyright 2018, Elsevier.
Conventionally, the process of heterogeneous electrocatalysis entails $\mathrm{CO}_{2}$ adsorption on the electrocatalyst surface, electron/mass transport to severe the $\mathrm{C}-\mathrm{O}$ bond with the generation of $\mathrm{C}-\mathrm{H}$ bonds, structural transformation and desorption of the reduced products from the electrocatalyst surface and subsequent diffusion into the electrolytic solution. ${ }^{363}$ The employed electrocatalyst and the applied electrode potential bias are one of the major factors that influence these processes and promote product selectivity.

From a thermodynamic point of view, a generally accepted CRR mechanism ( $\mathrm{pH} 7$ in aqueous solution (vs. SHE), $25{ }^{\circ} \mathrm{C}, 1$ atm, and $1 \mathrm{M}$ concentration of other solutes) for the primary products is illustrated below:

$$
\begin{aligned}
& \mathrm{CO}_{2}+2 \mathrm{H}^{+}+2 \mathrm{e}^{-} \rightarrow \mathrm{CO}+\mathrm{H}_{2} \mathrm{O}, E^{0}=-0.52 \mathrm{~V} \\
& \mathrm{CO}_{2}+2 \mathrm{H}^{+}+2 \mathrm{e}^{-} \rightarrow \mathrm{HCOOH}, E^{0}=-0.61 \mathrm{~V} \\
& \mathrm{CO}_{2}+4 \mathrm{H}^{+}+4 \mathrm{e}^{-} \rightarrow \mathrm{HCHO}+\mathrm{H}_{2} \mathrm{O}, \quad E^{0}=-0.51 \mathrm{~V} \\
& \mathrm{CO}_{2}+8 \mathrm{H}^{+}+8 \mathrm{e}^{-} \rightarrow \mathrm{CH}_{4}+2 \mathrm{H}_{2} \mathrm{O}, \quad E^{0}=-0.24 \mathrm{~V} \\
& \mathrm{CO}_{2}+12 \mathrm{H}^{+}+12 \mathrm{e}^{-} \rightarrow \mathrm{C}_{2} \mathrm{H}_{4}+4 \mathrm{H}_{2} \mathrm{O}, \quad E^{0}=-0.34 \mathrm{~V} \\
& \mathrm{CO}_{2}+6 \mathrm{H}^{+}+6 \mathrm{e}^{-} \rightarrow \mathrm{CH}_{3} \mathrm{OH}+\mathrm{H}_{2} \mathrm{O}, \quad E^{0}=-0.38 \mathrm{~V} \\
& 2 \mathrm{H}^{+}+2 \mathrm{e}^{-} \rightarrow \mathrm{H}_{2}, \quad E^{0}=-0.42 \mathrm{~V} \\
& \mathrm{CO}_{2}+\mathrm{e}^{-} \rightarrow \mathrm{CO}_{2}^{-}, \quad E^{0}=-1.9 \mathrm{~V}
\end{aligned}
$$

It is evident from reactions (43)-(48) that the CRR equilibrium potentials are analogous to that for the HER (reaction (49)). Realistically, this also corresponds with the formation of $\mathrm{H}_{2}$ as the principal by-product for the CRR process in aqueous solutions. Moreover, owing to the diminutive distinction between the thermodynamic potentials of each product (reaction (43)(48)), it is apparent that the selectivity of desired products is challenging. This is further compounded by the enormous energy (electrode potential) requirement to drive the CRR process, as indicated in reaction (50). ${ }^{364}$

This reaction (reaction (50)) is the first CRR step and incites the large overpotential required to activate the reaction processes. In this step, a key intermediate $\mathrm{CO}_{2}{ }^{--}$is formed by the first electron transfer to a $\mathrm{CO}_{2}$ molecule with a large overpotential of $-1.90 \mathrm{~V}$ ( $v s$. SHE) due to the energy required to bend the linear $\mathrm{CO}_{2}$ molecule to a radical anion. ${ }^{346}$ Subsequently, the formed $\mathrm{CO}_{2}{ }^{\bullet-}$ radical instantaneously reacts with several $\mathrm{H}^{+}$-coupled multiple-electron-transfer reactions to yield the reduced products. However, in practice, these intermediate reactions can be hindered as the OER occurs simultaneously with the CRR at the anode. To circumvent this, the cathode and anode compartments in the $\mathrm{CO}_{2}$ electrolytic cell are separated by means of an ion exchange membrane to avoid the oxidation of CRR products but promote the corresponding ion transfer. $^{365}$

4.1.2. Recent theoretical insight into CRR. The electroreduction of $\mathrm{CO}_{2}$ generally results in the synthesis of three basic products: $\mathrm{HCOOH} / \mathrm{HCOO}^{-}$, $\mathrm{CO}$ and other higher hydrocarbons such as $\mathrm{CH}_{4}, \mathrm{C}_{2} \mathrm{H}_{4}$, and $\mathrm{C}_{2} \mathrm{H}_{5} \mathrm{OH} .^{347,366}$ Based on this 
classification, theoretical insights into understanding the CRR mechanism are dependent on the specific reduced products.

Pertaining to the formation of formates or formic acids, theoretical investigations into the CRR over post-transition metals have been widely conducted. ${ }^{367,368}$ With regard to formate formation, studies have revealed that the oxide layer of metal oxides plays a significant role in the formate production, as illustrated in Fig. 8(b). ${ }^{369}$ Spectroscopic analysis supported by DFT calculations suggests that the initial steps of formate production arise from the formation of surface-bound carbonate or bicarbonate intermediates from the adsorption of $\mathrm{CO}_{2}$ on the surficial O-motifs or OH-motifs, respectively. ${ }^{367,370}$ Sequentially, the formed bicarbonate species is reduced to either ${ }^{*} \mathrm{COOH}$ or ${ }^{*} \mathrm{OCHO}$, with the latter being more thermodynamically favourable. ${ }^{371,372}$

Unlike some metals, the generation of formates does not occur via surface-bound carbonates/bicarbonates. A notable example is $\mathrm{Pb}$ electrodes where DFT calculations have suggested that formate formation proceeds by means of direct hydrogenation of $\mathrm{CO}_{2}$ by $\mathrm{H}$-adatoms. ${ }^{368,373}$ Likewise, theoretical and experimental investigation on Bi-based electrocatalysts confirms the absence of surface-bound carbonates toward the formation of formates over $\mathrm{Bi}$ dendrites. ${ }^{374}$ However, both studies agree that the ${ }^{*} \mathrm{OCHO}$ intermediate is more thermodynamically viable for formate generation. Moreover, Yoo and co-workers demonstrated using DFT calculations on several modelled metal surfaces that ${ }^{*} \mathrm{COOH}$ and ${ }^{*} \mathrm{H}$ are highly correlated in their free energies. Unlike the correlation between ${ }^{*} \mathrm{OCHO}$ and ${ }^{*} \mathrm{H}$, it is unlikely that the formation of formic acid will occur without the HER occurring along with. Based on the findings, the authors predicted that $\mathrm{Ag}$ and $\mathrm{Pb}$ are the most promising monometallic electrocatalysts with high $\mathrm{FE}$ for the production of $\mathrm{HCOOH}$ via CRR. ${ }^{375}$

Regarding the electrosynthesis of CO using CRR, several studies depict this reaction to proceed via the ${ }^{*} \mathrm{COOH}$ intermediate. ${ }^{340}$ In this case, two possible pathways are proposed. The first involves the single step proton-coupled electron transfer (PCET), while the second route is the formation of the $\mathrm{CO}_{2}{ }^{-}$- radical via single electron transfer and subsequent protonation to ${ }^{*} \mathrm{COOH} .{ }^{376}$ Fig. 8(c) illustrates the two mechanistic routes. Irrespective of the mechanistic route, theoretical studies have shown a good correlation in the binding energies between ${ }^{*} \mathrm{COOH}$ and ${ }^{*} \mathrm{CO}$, and ${ }^{*} \mathrm{H}$ and ${ }^{*} \mathrm{CO}$ on some metals, ${ }^{377}$ and between ${ }^{*} \mathrm{COOH}$ and ${ }^{*} \mathrm{CO}$, and ${ }^{*} \mathrm{COOH}$ and ${ }^{*} \mathrm{H}$ over a variety of other metals. $^{375}$ On this account, it is necessary that the development of electrocatalysts should consider schemes to optimize the surficial stabilization of ${ }^{*} \mathrm{COOH}$ without promoting the HER and influencing CO desorption. In this regard, further studies have revealed that the unsaturated coordinative sites such as the edges and corners are more promising sites for CO generation via $\mathrm{CRR}^{377,378}$ Experimental validation of this concept is demonstrated in the mechanistic studies on $\mathrm{Ag}^{379}$ and $\mathrm{Au} .{ }^{380}$ Here, the nanostructured catalyst systems possessed more active sites with unsaturated coordination than their metal foil analogues.

About the electrosynthesis of $\mathrm{C}_{2}$ products and other hydrocarbons via CRR (Fig. 8(d)), investigative studies on $\mathrm{Cu}$ and its compounds have been widely conducted. This has resulted in numerous proposed mechanisms, particularly for the different reduced products. ${ }^{381-388}$ Owing to this, it is unlikely to completely discuss each mechanistic insight without derailing from the scope of this study. Hence, this study will only review the theoretical insight for the electrosynthesis of $\mathrm{C}_{2} \mathrm{H}_{4}$. Kindly refer to the relevant literature above for further insights into other reduced products.

One of such mechanistic routes is the dimerization of ${ }^{*} \mathrm{CO}$, which was suggested to occur over the $\mathrm{Cu}(100)$ electrode. In this respect, studies have demonstrated both experimentally and computationally that the formation of $\mathrm{C}_{2} \mathrm{H}_{4}$ occurs shortly after the rate of $\mathrm{CO}$ generation has peaked. In addition, it was revealed that the onset potential for the generation of $\mathrm{C}_{2} \mathrm{H}_{4}$ is more negative when compared to that for CO. These findings were common on three different $\mathrm{Cu}(100,111$ and 110) surfaces. ${ }^{389}$ Subsequently, computational studies have revealed that ${ }^{*} \mathrm{CO}$ dimerisation is more favourable on the $\mathrm{Cu}(100)$ facets despite the commonality in the reaction dynamics. ${ }^{390}$ Moreover, $\mathrm{C}_{2} \mathrm{H}_{4}$ formation is hindered with an increase in CO coverage, which lowers the energy barrier. Compared to the $\mathrm{Cu}(111)$ facet, $\mathrm{Cu}(100)$ exhibited a favourable potential to synthesize $\mathrm{C}_{2}$ products from $\mathrm{CRR}$ due to the presence of under-coordinated $\mathrm{Cu}$ sites, which is likely to enable the $\mathrm{C}-\mathrm{C}$ coupling effect.

\subsection{Electrocatalysts of CRR}

It is established that the major parameters that hinder the industrial implementation of CRR are the selectivity, activity and stability, ${ }^{391}$ and each of these factors can be improved by focusing on the nature of the electrocatalyst, electrolytic solution and operating conditions. ${ }^{344}$ So far, the adopted CRR electrocatalysts include both heterogeneous and homogeneous metal based electrocatalysts consisting of earth-abundant TMs. ${ }^{361,362}$ Moreover, carbon-based electrocatalysts have also shown great potential as an efficient catalyst system for the electroreduction of $\mathrm{CO}_{2}$ to different reduced products. ${ }^{392}$

4.2.1 Metal electrocatalysts. Over time, heterogeneous metal electrocatalysts have been widely employed for the electrochemical reduction of $\mathrm{CO}_{2}$. The nature of these electrocatalysts has varied from their bulk form to nanostructures and from single atoms to $2 \mathrm{D}$ - and $3 \mathrm{D}$-electrocatalysts. In most cases, the formation of the key intermediate $\mathrm{CO}_{2}{ }^{-}$is considered as the rate-determining step for electrochemical reduction of $\mathrm{CO}_{2}$. Hence, the employed electrocatalyst should enable the stabilization of this intermediate to attain high CRR performance.

Previously, research studies have demonstrated that depending on the reaction intermediates being formed and the final reduced products, crystalline bulk metals are classified into three: (i) earth-abundant TMs (such as $\mathrm{Zn}, \mathrm{Sn}, \mathrm{Pb}$ and $\mathrm{Bi})$ that can generate $\mathrm{HCOOH} / \mathrm{HCOO}^{-}$via the outer-sphere mechanism as a result of the weak binding with the $\mathrm{CO}_{2}{ }^{\bullet-}$ intermediate (Fig. 8(b)); (ii) noble-metal based catalyst systems such as $\mathrm{Au}, \mathrm{Ag}$ and $\mathrm{Pd}$ that have a strong affinity towards the ${ }^{*} \mathrm{COOH}$ intermediate resulting in its further reduction to generate the weakly bound ${ }^{*} \mathrm{CO}$ intermediate. Subsequently, CO is 
desorbed from the surface emerging as the main product (Fig. 8(c)); ${ }^{361}$ (iii) Cu-based electrocatalysts which have demonstrated to be the only catalyst system to bind and transform the ${ }^{*} \mathrm{CO}$ intermediate into other products. ${ }^{393-395}$ In order words, Cu is the only catalyst system with the potential to facilitate CRR involving more than two electrons $\left(2 \mathrm{e}^{-}\right)$transfer with significant FE. ${ }^{396}$ Specifically, at low and high overpotentials, $2 \mathrm{e}^{-}$transfer products (such as $\mathrm{H}_{2}, \mathrm{CO}, \mathrm{HCOOH}$ ) and multi $\mathrm{e}^{-}$transfer products (such as $\mathrm{CH}_{4}, \mathrm{C}_{2} \mathrm{H}_{4}$ ) are generated, respectively.

As witnessed in previous sections, suppressing the HER has been one of the focal points of most electrochemical reduction of small molecules. Similarly, attention towards retarding this reaction process is also necessary to achieve high selectivity of CRR products. On this account, schemes are employed to enhance the catalytic activity of catalyst systems, which can be reached by utilizing metal alloys, metal oxides, nanostructured metals and chalcogenides that offer enriched active centers for $\mathrm{CO}_{2}$ adsorption and activation. ${ }^{397-399}$ For instance, utilizing nanostructured $\mathrm{Ag}$ oxide as a CRR electrocatalyst has demonstrated a high CO selectivity of about $80 \%$ ( $0.49 \mathrm{~V}$ overpotential), which is considerably higher than the $4 \%$ selectivity of the $\mathrm{Ag}$ alone catalyst under similar conditions. The improved activity and selectivity were attributed to the strong ${ }^{*} \mathrm{COOH}$ stability on the active sites of the metal oxide. ${ }^{400}$

Most importantly, experimental evidence has shown that the surface nature, morphology and size of the catalyst system are influential towards the product distribution over the electrocatalysts. For instance, amorphous Cu NPs have been proven to have superior CRR activity and selectivity towards $\mathrm{HCOOH}$ and $\mathrm{C}_{2} \mathrm{H}_{5} \mathrm{OH}$ over the crystalline counterpart with $37 \%$ and $22 \% \mathrm{FE}$, respectively, at $-1.4 \mathrm{~V}(v s$. $\mathrm{Ag} / \mathrm{AgCl})$. The improved performance was ascribed to the enriched defective sites as a result of irregularity in the surface structure in the amorphous form (Fig. 9(a) and (b)). ${ }^{401}$ Sequel to this, Hwang and co-workers adopted a mix of $\mathrm{Cu}$ states in anodized $\mathrm{Cu}(\mathrm{AN}-\mathrm{Cu})$ as a more stable and highly selective CRR electrocatalyst for the generation of $\mathrm{C}_{2} \mathrm{H}_{4}$. The improved selectivity of $\mathrm{C}_{2} \mathrm{H}_{4}$ over $\mathrm{CH}_{4}$ was attributed to the electrochemical reduction environment enabled by the mixed valence of the $\mathrm{O}-\mathrm{Cu}$ combination catalysts (Fig. 9(c) and (d)). ${ }^{399}$ Likewise, Rosa M. et al. tuned copper's morphology and oxidation state by pulsed $\mathrm{CO}_{2}$ electrolysis and the production of $\mathrm{C}_{2+}$ products was enhanced with $76 \% \mathrm{FE}$ at $-1.0 \mathrm{~V}$ (vs. RHE). According to quasi in situ XPS results, they found that the improved efficiency of the $\mathrm{Cu}$ catalyst was due to the cooperation of $\mathrm{Cu}(\mathrm{I})$ species and continuous regeneration of defects which would promote $\mathrm{C}-\mathrm{C}$ coupling pathways. ${ }^{402}$

Relative to single metals, hybrid or alloy metals have shown to offer improved CRR performance due to the potential to modulate the binding energy of specific intermediates on the catalyst surface. ${ }^{403,404}$ For instance, Hoang and co-workers demonstrated the synthesized $\mathrm{CuAg}$ alloy film to be a more stable and efficient CRR electrocatalyst for $\mathrm{C}_{2} \mathrm{H}_{4}$ and $\mathrm{C}_{2} \mathrm{H}_{5} \mathrm{OH}$ production with a $\mathrm{FE}$ reaching nearly $60 \%$ and $25 \%$, respectively, at $-0.7 \mathrm{~V}$ (vs. RHE). ${ }^{404}$ Elsewhere, it was elucidated that the integrating $\mathrm{Ag}$ and $\mathrm{Cu}$ atoms resulted in the occurrence of compressive strain around $\mathrm{Cu}$ atoms that promoted the product selectivity. ${ }^{405}$ Given the tunable effect of $\mathrm{Cu}$ towards the bonding of CO-motifs and selective reduction of other intermediates, several Cu-based hybrids or alloys are developed for efficient CRR electrocatalysts. ${ }^{405-407}$

Most importantly, it is worth mentioning that despite the beneficial effects of these metals on the CRR performance, the activity and selectivity can be significantly influenced by the metal content. For instance, $\mathrm{Ma}$ and co-workers revealed that the product selectivity during the electroreduction of $\mathrm{CO}_{2}$ using $\mathrm{Cu}$-based alloys is highly affected by the $\mathrm{Cu}$ content. ${ }^{408}$ Therefore, an optimization of the metal content is required for an optimal performance of $\mathrm{CO}_{2}$ conversion. In addition, investigative studies towards the discovery of alloys with a suitable coupling effect in order to promote efficient and highly selective CRR performance are encouraged. To this effect, Zhang and co-workers examined the CRR activity of several Sn-based bimetallic catalysts towards the formation of $\mathrm{HCOOH}$. The experimental results demonstrated that $\mathrm{Ag}-\mathrm{Sn}$ and $\mathrm{Cu}-\mathrm{Sn}$ are the most favourable for $\mathrm{HCOOH}$ production with a $\mathrm{FE}$ of $88.3 \%$ and $87.4 \%$, respectively, when tested in $0.5 \mathrm{M} \mathrm{NaHCO}_{3} .{ }^{409}$

In addition to $\mathrm{Cu}$, other earth-abundant TMs (such as $\mathrm{Ni}$, Co, Zn, Bi and In) and their compounds have demonstrated remarkable potential as CRR electrocatalysts. ${ }^{71,339,410,411}$ For instance, Pan and co-workers demonstrated that an engineered Co- $\mathrm{N}_{5}$ catalytic site using a modified Stöber method is a highly efficient CRR electrocatalyst for CO production with $\mathrm{FE}>99 \%$ at -0.73 and $-0.79 \mathrm{~V}$ vs. RHE (Fig. 9(h)). ${ }^{412}$ Similarly, a carbonanchored N-derived $\mathrm{Zn}$ catalyst $\left(\mathrm{ZnN}_{4} / \mathrm{C}\right)$ demonstrated high CRR selectivity and stability towards CO production with a FE of $\sim 95 \%$ and $>75 \mathrm{~h}$ stability at an onset overpotential of $24 \mathrm{mV}^{413}$ And $\mathrm{FeN}_{4}$ sites in $\mathrm{Fe}-\mathrm{N}-\mathrm{C}$ catalysts were also obtained from the ZIF-8 which achieved $25 \mathrm{~mA} \mathrm{~cm}{ }^{-2}$ at $0.8 \mathrm{~V}$ (vs. RHE) and FE was above $90 \%$ for CO in a wide potential. ${ }^{414}$ For these electrocatalysts, experimental and theoretical results reveal that the $\mathrm{Zn}-\mathrm{N}_{4}, \mathrm{Co}-\mathrm{N}_{5}$ and $\mathrm{Fe}-\mathrm{N}_{4}$ catalytic sites facilitated the activation of $\mathrm{CO}_{2}$ and direct $\mathrm{COOH}^{*}$ formation. However, the product formed via In-based catalysts is different from those of $\mathrm{Fe}$, Co, and $\mathrm{Zn}$ based electrocatalysts which would convert $\mathrm{CO}_{2}$ to formate. Recently, Yin's group synthesized In-N-C via In-doped ZIF-8 and the atomically dispersed structure demonstrated a high CRR performance with a turnover frequency of $26771 \mathrm{~h}^{-1}$ at $-0.99 \mathrm{~V}$ ( $v s$. RHE) and the maximum $\mathrm{FE}$ for formate was around $80 \%$. Since In was atomically dispersed in the structure, the intermediate ${ }^{*} \mathrm{OCHO}$ was formed on isolated In sites which affected the formate formation. ${ }^{339}$

Furthermore, to enrich the distribution of active sites, enabling a large catalyst surface area can feature catalytic active centers to improve the CRR performance. On this account, a myriad of 2D materials including nanosheets and nanofilms of metals, metal oxides and chalcogenides have portrayed good CRR activity and selectivity. ${ }^{415-417}$ The versatility of $2 \mathrm{D}$ electrocatalysts originates from their unique electronic structure and stability. In addition, they offer the beneficial features of both 

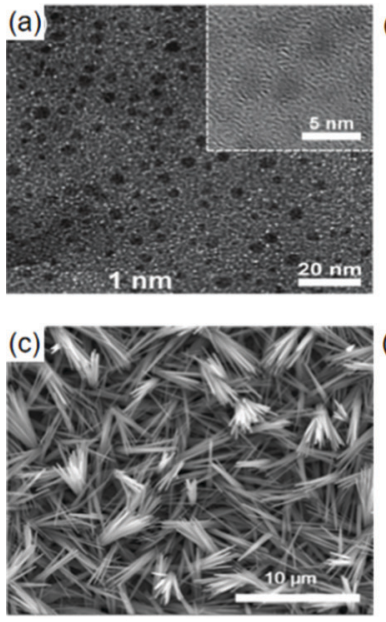

(b)

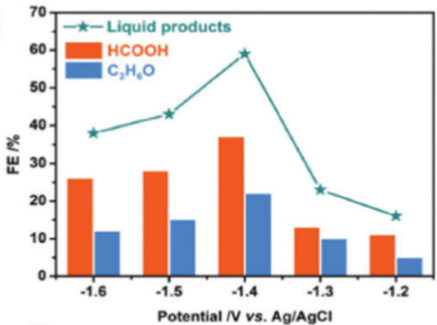

(d)

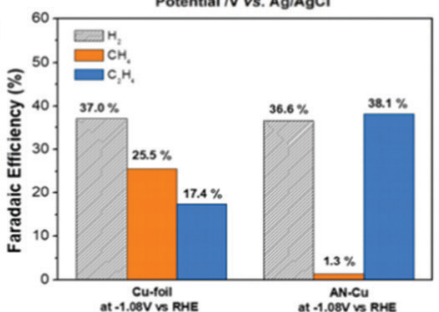

(f)
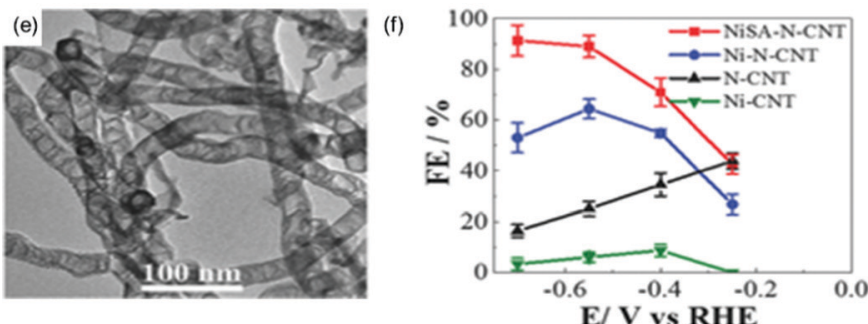

E/ V vs RHE
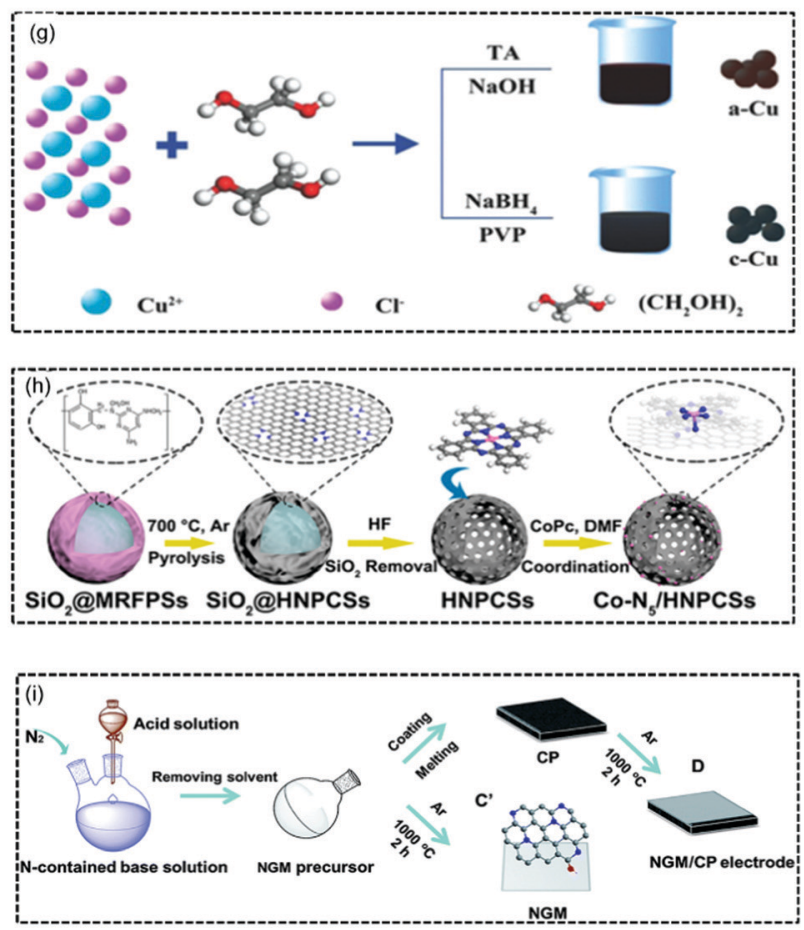

Fig. 9 (a) TEM image of amorphous Cu NPs and their (b) FE for liquid products at each given potential for $2 \mathrm{~h}$. Panels (a) and (b) are reproduced with permission. ${ }^{401}$ Copyright 2018, Wiley. (c) HRTEM image of $\mathrm{Cu}(\mathrm{AN}-\mathrm{Cu}$ ) and its (d) FE for HER and hydrocarbon selectivity in comparison to Cu-foil. Panels (c) and (d) are reproduced with permission. ${ }^{399}$ Copyright 2018, American Chemical Society. (e) TEM image of NiSA-N-CNTs. (f) Faradaic efficiency of CO for NiSA-N-CNTs, Ni-N-CNTs, N-CNTs, and Ni-CNTs at $-0.28,-0.40,-0.55$, and $-0.70 \mathrm{~V}$. Panels (e) and (f) are reproduced with permission. ${ }^{413}$ Copyright 2018, Wiley. (g) Schematic illustration of the formation process of a-Cu and c-Cu. ${ }^{401}$ Copyright 2018, Wiley. (h) Schematic illustration of the formation of Co- $\mathrm{N}_{5} / \mathrm{HNPCSs}{ }^{412}$ Copyright 2018, American Chemical Society. (i) Schematic illustration of the procedures to prepare NGM and NGM/CP electrodes. ${ }^{348}$ Copyright 2016, Royal Society of Chemistry.

heterogeneous and molecular electrocatalysts. ${ }^{418,419}$ For instance, Gao and co-workers evaluated the HCOO formation potential during CRR on two different Co-based catalytic sites. The authors revealed that surface Co atoms on the atomically thin layers displayed a higher activity and selectivity at lower overpotentials than surface Co atoms on the bulk samples. The improved activity was due to the partial oxidation of the atomic layers leading to a stable current density of $10 \mathrm{~mA} \mathrm{~cm} \mathrm{~cm}^{-2}$ and a $\mathrm{FE}$ of $90 \%$ at $0.24 \mathrm{~V}$ (vs. RHE) with $40 \mathrm{~h}$ stability. ${ }^{386}$ And very recently, Cao et al. fabricated thin bismuthene (Bi-ene) with a few layers which displayed a high selectivity with $\mathrm{FE}$ of nearly $100 \%$ from $-0.83 \mathrm{~V}$ to $-1.18 \mathrm{~V}$ (vs. RHE). Based on DFT analysis and the result of in situ ATR-IR spectra, the product formate was finally obtained from the $\mathrm{OCHO}^{*}$ intermediate. ${ }^{417}$

Moreover, tailoring the intrinsic electronic structure of the electrocatalyst can benefit the CRR selectivity. Here, $\mathrm{Xu}$ and co-workers revealed that the partial charge delocalization in the MoSeS monolayer resulted in the tuning of the d-band electronic structure by the lengthened Mo-Se and shortened Mo-S bonds. This alteration in the electronic structure favoured the stabilization of the $\mathrm{COOH}^{*}$ intermediate and facilitated the $\mathrm{CO}$ desorption step, which was considered as the rate-determining step. Based on this finding, the MoSeS monolayer achieved a remarkably high $\mathrm{FE}$ of $45.2 \%$ for the CRR towards CO formation, when compared to independent chalcogenides, $\mathrm{MoSe}_{2}$ and $\mathrm{MoS}_{2}$ monolayers, with $30.5 \%$ and $16.6 \%$, respectively at $-1.15 \mathrm{~V} v s$. RHE. ${ }^{420}$

4.2.2. Molecular electrocatalysts. The application of molecular electrocatalysts for CRR has been recently investigated with emphasis on noble metals (such as $\mathrm{Pd}, \mathrm{Re}$ and $\mathrm{Ru}$ ) and earth-abundant TMs (such as Co, Fe, Mn and Ni). ${ }^{339,362,421,422}$ Here, the CRR process is triggered by the potential-induced change in the oxidation state of the metals or the incorporated ligands. ${ }^{344}$ However, this depends on the metal-ligand cooperativity, which has been interestingly favoured by the metals mentioned above. Hence, the metal-ligand interaction has become a fundamental factor for regulating the catalytic behavior of molecular electrocatalysts. Several molecular electrocatalysts have been developed for the CRR process. Recent developments on this, their applications and mechanistic investigations are reviewed in the literature. ${ }^{361}$ Despite the wide range of molecular electrocatalysts developed for the production of $\mathrm{CO}$ and $\mathrm{HCOOH} / \mathrm{HCOO}^{-}$, extensive studies focusing on the efficient production of the multi $\mathrm{e}^{-}$-transfer $\left(>2 \mathrm{e}^{-}\right)$products such as $\mathrm{HCHO}, \mathrm{CH}_{3} \mathrm{OH}$, and $\mathrm{CH}_{4}$ are limited. An example of a commonly applied molecular electrocatalyst for the electroreduction of $\mathrm{CO}_{2}$ is the polypyridyl TM complexes. ${ }^{421}$ Generally, this set of electrocatalysts possess high CRR efficiency towards CO 
generation with more promising activity in aprotic solvents. Besides, given the low solubility of $\mathrm{CO}_{2}$ in aqueous solutions these brands of electrocatalysts are seldom used in aqueous electrolytes. $^{423}$

Another major disadvantage of molecular electrocatalysts is the poor recyclability and stability of the catalyst systems. ${ }^{424}$ Exploiting recent advancements in ligand mobilization, newly developed hybrid catalysts anchored on conductive supports have demonstrated more extended CRR stability. ${ }^{425,426}$ For instance, Wang and co-workers appended the $\mathrm{Co}^{\text {II }}$ quaterpyridine complex [Co(qpy) $]^{2+}$ on the surface of multi-walled CNTs to catalyze the electroreduction of $\mathrm{CO}_{2}$ to generate $\mathrm{CO}$ in water at $\mathrm{pH}$ 7.3. Experimental results revealed that the hybrid complex attained $100 \%$ selectivity and $100 \% \mathrm{FE}$ with a current density of $0.94 \mathrm{~mA} \mathrm{~cm} \mathrm{~cm}^{-2}$ at $-0.35 \mathrm{~V}$ (vs. RHE). Moreover, a current density of $9.3 \mathrm{~mA} \mathrm{~cm} \mathrm{~cm}^{-2}$ at an overpotential of only $340 \mathrm{mV}$ was sustained for 89095 catalytic cycles. ${ }^{426}$

In addition to the activity and selectivity, it is also pertinent to have an in-depth understanding of the mechanistic CRR pathway on each molecular electrocatalyst. A detailed insight into this is well documented in the literature. ${ }^{421}$ Generally, the CRR mechanism can be investigated by studying the formation of intermediates using quantum chemical simulations along with electroanalytical tools and spectroscopy (such as Fourier transform infrared spectroscopy (FTIR)-spectroelectrochemistry (SEC) and UV-vis-SEC). ${ }^{361,421}$

4.2.3. Carbon-based electrocatalysts. Given the advantages of carbon-based electrocatalysts as stipulated in previous sections, this set of catalytic materials are also considered for catalysing the electrochemical reduction of $\mathrm{CO}_{2} \cdot{ }^{355,427}$ Among the most prominent features of these electrocatalysts, the enabled assembly of the $\mathrm{C}$ atoms into diverse structures and dimensions with high chemical and mechanical strength differentiates them from others. ${ }^{348}$ On this account, common carbon materials with intrinsic properties to support the CRR include CNTs, carbon nanofibers (CNFs), nanoporous carbon, graphene, diamond and graphene dots. More importantly, these catalysts are also doped with heteroatoms such as $\mathrm{N},{ }^{428,429} \mathrm{~B},{ }^{430,431} \mathrm{P},{ }^{432,433} \mathrm{~S}^{434}$ and $\mathrm{F}^{403}$ to further enhance the CRR activity and selectivity. ${ }^{392}$

The electrocatalytic performance of the heteroatom-doped carbon-based catalyst systems partially depends on the electronic structure of the heteroatoms in comparison to the positively charged $\mathrm{C}$ atoms.

In this case, the selectivity of a specific reduced product strongly depends on the active site's affinity towards the corresponding intermediate motifs. This is quite different from the conventional pristine carbon materials. Specifically, CRR reduced products involving the $2 \mathrm{e}^{-}$-transfer $\left(\mathrm{CO}\right.$ and $\mathrm{HCOO}^{-}$) are typically generated over the carbon-based electrocatalyst. However, other products involving multiple $\mathrm{e}^{-}$-transfer are also formed over the carbon-based electrocatalyst but with a particular composition and/or morphology.

Concerning heteroatom N-dopants, the activation of the CRR over carbon-based electrocatalysts occurs on the N-dopant. ${ }^{435-437}$ Sun and co-workers revealed that the N-motif on the $\mathrm{N}$-doped carbon electrode enhanced the catalytic activity of the graphenelike carbon with high selectivity for $\mathrm{CH}_{4}(93.5 \%)$ at $-1.4 \mathrm{~V} v$ s. SHE (Fig. 9(i)). ${ }^{348}$ Similarly, Duan and co-authors revealed by both theoretical and experimental studies that the pyridinic N-dopant is the most active site for the electro-reduction of $\mathrm{CO}_{2}$ to $\mathrm{CO}^{392}$ More importantly, incorporating $\mathrm{N}$-species into the carbon framework of the electrocatalyst has also been demonstrated to favour specific product selectivity. Wu and co-workers synthesized $\mathrm{C}_{2} \mathrm{H}_{4}$ for the first time as the major product from the CRR over a metalfree electrocatalyst. Here, $31 \% \mathrm{FE}$ was achieved at a potential of $-0.75 \mathrm{~V}$ ( $v s$. RHE) over N-doped graphene quantum dots (NGQDs) in $1 \mathrm{M} \mathrm{KOH}^{438}$

Another example involves the generation of $\mathrm{C}_{2} \mathrm{H}_{5} \mathrm{OH}$ from the electroreduction of $\mathrm{CO}_{2}$ over metal-free $\mathrm{N}$-doped mesoporous carbon with a high $\mathrm{FE}$ of $77 \%$ at $-0.56 \mathrm{~V}$ ( $v s$. RHE). As mentioned earlier, the morphology of the electrocatalyst also plays a significant role in the CRR catalytic activity. In this study, Song and co-workers also demonstrated that the cylindrical structure of the $\mathrm{N}$-doped mesoporous carbon enhanced the $\mathrm{C}-\mathrm{C}$ coupling effect, which resulted in the high selectivity for $\mathrm{C}_{2} \mathrm{H}_{5} \mathrm{OH}$ while suppressing the $\mathrm{CO}$ formation with the potential range of -0.4 to $-1.0 \mathrm{~V}$. Also, experimental verification depicts that the cylindrical construct of the electrocatalyst aided the easy transport of electrons which is responsible for the enhanced $\mathrm{C}-\mathrm{C}$ coupling effect. ${ }^{439}$ Besides N-dopants, other common dopants employed to improve the CRR performance of carbon-based electrocatalysts include $\mathrm{B},{ }^{430,440} \mathrm{P},{ }^{432,433} \mathrm{~S}^{434}$ and $\mathrm{F}^{403}$ Detailed description of the CRR activity of these catalysts and other notable CRR electrocatalysts is summarized in Table 3.

Aside from the metal-free dopants, metal-doping on the carbon-based electrocatalysts has also been studied. ${ }^{441-443}$ For instance, Cheng and co-workers investigated a class of TMs that were atomically dispersed on N-doped CNTs (MSA-N-CNTs, where $\mathrm{M}=\mathrm{Ni}$, Co, NiCo, CoFe, and NiPt) using a new multistep pyrolysis approach. Among these materials, NiSA-N-CNTs demonstrated the most suitable CRR activity and selectivity towards CO production, achieving a turnover frequency (TOF) of $11.7 \mathrm{~s}^{-1}$ and an FE of $89 \%$ at $-0.55 \mathrm{~V}$ ( $v s$. RHE), with FE being two orders of magnitude higher than that of Ni nanoparticles supported on CNTs (Fig. 9(e) and (f)). ${ }^{413}$

Moreover, it was shown that the introduction of metal-based atoms could enhance both the efficiency and selectivity of the CRR process towards the production of $\mathrm{C}_{2}$ hydrocarbons. Jiao and co-workers proposed a molecular scaffolding approach for synthesizing a carbon-based complex with synergistic active sites to promote the $\mathrm{CRR}$. It was underscored that $\mathrm{Cu}$, probed on graphitic carbon nitride $\left(\mathrm{g}-\mathrm{C}_{3} \mathrm{~N}_{4}\right)$, served as a molecular scaffold to regulate the electronic structure of $\mathrm{Cu}$. Compared to the $\mathrm{Cu}(111)$ surface, the prepared $\mathrm{Cu}-\mathrm{C}_{3} \mathrm{~N}_{4}$ complex is enriched with active centers that enabled $\mathrm{CO}_{2}$ activation and further reduction to generate $\mathrm{C}_{2}$ products. Theoretical evidence relates the good catalytic performance of the complex to the strong affinity of C-bound and O-bound intermediates to $\mathrm{Cu}$ and $\mathrm{g}-\mathrm{C}_{3} \mathrm{~N}_{4}$, respectively. ${ }^{447}$

In summary, CRR reduces $\mathrm{CO}_{2}$ which is different from NRR that reduces $\mathrm{N}_{2}$, but the perspective strategies as 
Table 3 Summary of the performances of CRR electrocatalysts

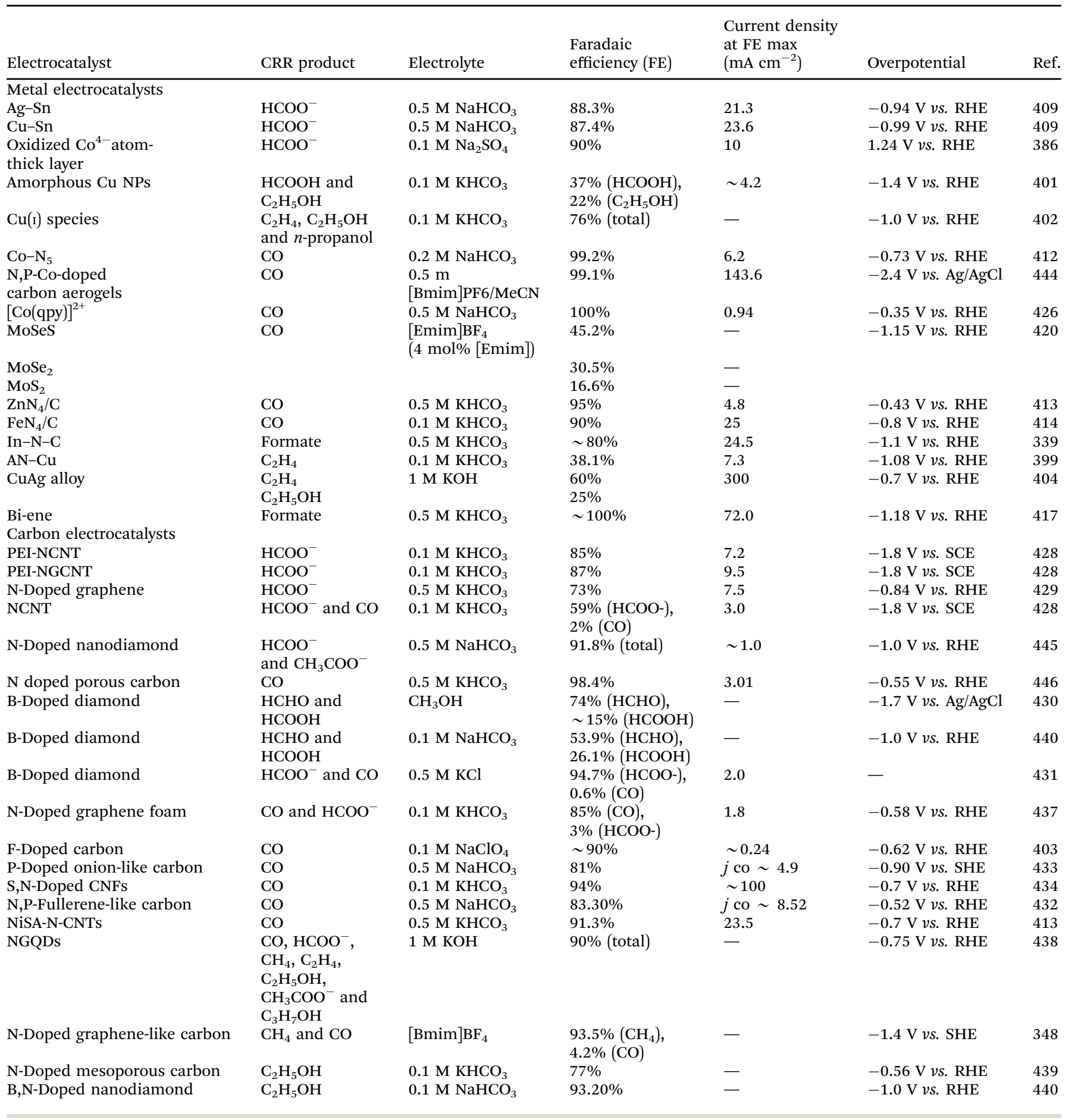

discussed above for NRR are still laterally referenceable for CRR. Additionally, the unique characteristic for CRR lies in its product diversity, including $\mathrm{C}_{1}, \mathrm{C}_{2}$ and $\mathrm{C}_{3+}$ carbon based products. This proffers unlimited research space, while bringing with complex challenges at the same time. From this point, the in situ microscopy and spectroscopy techniques will offer the robust research tools for CRR to better explore the intermediates during redox reactions and hence to enable the control on customized reaction pathways for target carbon products.

\section{Conclusion and outlook}

In recent years, the prospective goal to develop sustainable pathways for fuels and chemicals has given rise to the 
advancements in electrocatalyst design and development. In this review, we extensively discussed the recent advances in electrocatalysis-enabled transformation of earth-abundant water, nitrogen and carbon dioxide with the sustainable goal of establishing environmentally sensible circulation of energy and materials. ${ }^{44,448,449}$

First, the fundamental principles, mechanistic pathways, recent theoretical concepts and the energetics underlying each electrocatalytic reaction (e.g. water splitting, $\mathrm{N}_{2}$ reduction reaction and $\mathrm{CO}_{2}$ reduction reaction) were initially studied to reveal the common and distinct challenges. Second, the performances of known catalyst systems pertaining to each reaction were discussed, reflecting on the different outcomes and mechanistic understanding of catalyst design principles for developing enhanced electrocatalysts. Specifically, the current status of available metal and metal-free electrocatalysts for the reactions based on a common set of figures of merit, namely yield rate, faradaic efficiency, overpotential, current density, and stability, was explored in detail. In addition, it was found that the practical implementation of each reaction and its electrocatalysts known to date is hindered by the occurrence of different adsorbed intermediates and the accompanying energies, which alters the catalytic activity and stability. For instance, the poor performance of a myriad of NRR electrocatalysts is due to the occurrence of HER which competitively consumes abundant electrons and protons to form $\mathrm{H}_{2}$ against the formation of $\mathrm{NH}_{3}$. This circumstance intensified the challenge in the limited depth of material knowledge and scale-up dynamics of current catalyst-development strategies. Accordingly, a new strategy in the development of catalyst systems is required to counter these constraints with a special emphasis on modulating the catalytic efficiency, selectivity and stability. Moreover, another design strategy is to construct a self-supported 3D catalyst architecture that promotes larger number of active sites and improves electrical contact. This approach may include doping, chemical functionalization, alloying or defect introduction, as well as the synthesis method and conditions. Furthermore, the need to elucidate the kinetics and reaction barriers at the electrolyteelectrode interface, together with the modalities of electron/ proton transfer cannot be over-exaggerated. In this regard, one of the main conclusions is that an integrated scheme is required to strengthen both experimental and theoretical insightful tools towards the design, synthesis, characterization and testing of practical catalyst systems. In spite of significant progress made as discussed above, there are still many challenges ahead in the development of electrocatalysts for overall water splitting, $\mathrm{CO}_{2}$ reduction and $\mathrm{N}_{2}$ reduction reactions and further efforts are also required to elucidate other factors that can expedite the advancements. Prospective research studies in this regard can focus on the following points.

(1) An in-depth understanding of the related mechanism for each reaction is critically sought. This would in turn provide a knowledge-driven scheme for the design and development of efficient catalysts by optimizing computational studies towards the reaction mechanism. Specifically, investigation into the mechanism can guide structural modification, electronic reconfiguration and prevention of catalyst active site degradation during cycling. In addition, theoretical calculations can discern at an atomic level the competitive interactions hindering high yield in each reaction, e.g. the competitive interaction between NRR and HER in the electrocatalytic synthesis of ammonia. Most reported computational studies are based on simplified models and hence lack accurate prediction of the actual kinetics and reaction mechanisms under given operating conditions.

(2) Morphology-engineered catalyst systems have demonstrated high performance efficacy and stability in the considered electrocatalytic processes. ${ }^{450,451}$ On this account, the catalyst with abundant active sites can be fabricated into specific configurations (such as layers, 3D, nanowires and nanotubes) in order to improve the catalyst's physiochemical properties. By doing so, the porosity and number of accessible active sites are increased, hence facilitating species adsorption, activation and electron diffusion. For instance, heteroatom doping and modulation of the composition of the catalyst alter the electronic structure of active sites so as to optimize the intrinsic activity of bifunctional HER-OER catalysts. In the case of NRR, the catalytic activity is highly dependent on the transfer of electrons, which was observed to be more effective in some special structures such as sharp spikes known to concentrate the electric field at the spikes. Hence, it is necessary to optimize the structure with respect to the morphology to end up in enhanced catalysis.

(3) Generally, extensive integration of computational and experimental studies in the design of catalyst systems is lacking, particularly in the fabrication methods for the engineered catalysts. Most reported studies in this regard are theory-based with little or no detailing of experimental schemes to effectuate the newly developed catalyst active sites. Ideally, to integrate all mechanistic information demands a rigorous standardization of experimental setups and procedures, in-depth understanding beyond surficial catalyst interactions and a multi-scale modeling involving all these aspects.

(4) Thorough knowledge-based studies on the above outlooks are essential mainly for the development of novel or improved electrocatalysts. Thereupon, the development of functional composites with better catalytic activity and stability is not far-fetched. ${ }^{452}$ For instance, various heteroatom-doped functional carbon-based materials have displayed exceptional potential towards overall water splitting due to their tunable structure, available active sites and durability in alkaline/acidic electrolytes. Another example is the adoption of the zeolitic imidazolate encapsulated catalyst, which has the potential to suppress HER in the NRR process by absorbing H-atoms. In summary, the optimization of these advanced functional materials is vital for the practical application of these processes.

(5) In addition to the optimization of composite catalysts, measures can be adopted to expedite this process via accelerating catalyst discovery. Due to advancements in machine learning and material genome databases, accelerating catalyst discovery by high-throughput assessment and non-supervised analytical 
techniques such as AI algorithms, aided with the identification of key synthetic parameters, is realistic. ${ }^{453,454}$ Moreover, the state-ofthe-art computer-aided robotic and automated facilities enable autonomous catalyst synthesis, characterization and performance evaluation, which could significantly boost the discovery of advanced catalysts for electrochemical conversion of water, nitrogen, carbon dioxide and the other molecules. ${ }^{455}$ This critical review with more than 500 references along with groups of expertise helped to lay the foundation in this research field.

(6) Although optimizing the operating cell was not covered in detail in this study, it is worth mentioning here for future research studies. The practical application of the electrocatalytic conversion of earth-abundant molecules goes beyond the understanding of the surficial interactions on the electrocatalysts. Knowledge of the electrolytic cells and optimal operating conditions is equally vital. For instance, $\mathrm{NH}_{3}$ is theoretically reduced and detected at the cathode. However, this is not the case practically as a significant amount of $\mathrm{NH}_{3}$ is observed to crossover to the anode, which could be oxidized and subsequently reduce the yield of $\mathrm{NH}_{3}$. Therefore, the need for the design and optimization of the cell is necessary to circumvent such occurrences.

(7) In view of the technical advancement in this field, researchers underscore the importance of developing more resolute legal and techno-economic frameworks that can successfully promote sustainable solutions by internalizing environmental costs. Overall, a level of parallelism between the technological, economic and legal aspects of these technologies at the initial stage of development and coordinated efforts based on a long-term view should be established. This is valuable for the practical exploitation and commercial extension of these electrochemical conversion processes. However, despite the promising laboratory-based efficiencies, particularly for the most important reaction (HER), the processes are still long way away from practical application. Recent advancements lack feasibility tests on a pilot scale for the highly efficient electrocatalysts designed to date. In addition, it is envisaged that even with the accelerated advancement in electrocatalyst development, conventionally manufactured electrocatalysts may likely exhibit a better economy of scale, hence discouraging the implementation of sensible catalyst schemes.

\section{Conflicts of interest}

There are no conflicts to declare.

\section{Acknowledgements}

This work was supported by the National Natural Science Foundation of China (52173234, 22102208), Shenzhen-Hong Kong-Macau Technology Research Programme (Type C, SGDX2020110309300301), Shenzhen Science and Technology Program (JCY20210324102008023), Shenzhen Excellent Science and Technology Innovation Talent Training Project - Outstanding Youth Project (RCJC20200714114435061), the ANU Futures
Scheme (Q4601024), CCF-Tencent Open Fund and Functional Materials Interfaces Genome (FIG) project.

\section{References}

1 S. Chen, S. S. Thind and A. Chen, Electrochem. Commun., 2016, 63, 10-17.

2 Z. J. Schiffer and K. Manthiram, Joule, 2019, 1, 10-14.

3 Z. W. Seh, J. Kibsgaard, C. F. Dickens, I. Chorkendorff, J. K. Nørskov and T. F. Jaramillo, Science, 2017, 355, eaad4998.

4 F. Naseem, P. Lu, J. Zeng, Z. Lu, Y. H. Ng, H. Zhao, Y. Du and Z. Yin, ACS Nano, 2020, 14, 7734-7759.

5 J. Hwang, R. R. Rao, L. Giordano, Y. Katayama, Y. Yu and Y. Shao-Horn, Science, 2017, 358, 751-756.

6 A. J. Martín and J. Pérez-Ramírez, Joule, 2019, 3, 2602-2621.

7 H. Lu, J. Tournet, K. Dastafkan, Y. Liu, Y. H. Ng, S. K. Karuturi, C. Zhao and Z. Yin, Chem. Rev., 2021, 121, 10271-10366.

8 W. Kreuter and H. Hofmann, Int. J. Hydrogen Energy, 1998, 23, 661-666.

9 J. Jordan and P. T. Smith, Proc. Chem. Soc., 1960, 246-247.

10 S. Meshitsuka, M. Ichikawa and K. Tamaru, J. Chem. Soc., Chem. Commun., 1974, 158-159.

11 Y. Hori, A. Murata and R. Takahashi, J. Chem. Soc., Faraday Trans. 1, 1989, 85, 2309-2326.

12 H. Yoshio, K. Katsuhei and S. Shin, Chem. Lett., 1985, 15, 897-898.

13 H. Yoshio, K. Katsuhei, M. Akira and S. Shin, Chem. Lett., 1986, 897-898.

14 N. Furuya and H. Yoshiba, J. Electroanal. Chem. Interfa. Electrochem., 1990, 291, 269-272.

15 J. Liu, D. Zhu, Y. Zheng, A. Vasileff and S.-Z. Qiao, ACS Catal., 2018, 8, 6707-6732.

16 M. S. Dresselhaus and I. L. Thomas, Nature, 2001, 414, 332-337. 17 J. A. Turner, Science, 2004, 305, 972.

18 Q. Liu, Z. Pu, A. M. Asiri, A. H. Qusti, A. O. Al-Youbi and X. Sun, J. Nanopart. Res., 2013, 15, 2057.

19 J. Tian, N. Cheng, Q. Liu, W. Xing and X. Sun, Angew. Chem., Int. Ed., 2015, 54, 5493-5497.

20 M. G. Walter, E. L. Warren, J. R. McKone, S. W. Boettcher, Q. Mi, E. A. Santori and N. S. Lewis, Chem. Rev., 2010, 110, 6446-6473.

21 M. Gong, D.-Y. Wang, C.-C. Chen, B.-J. Hwang and H. Dai, Nano Res., 2016, 9, 28-46.

22 Y. Wu, M. Chen, Y. Han, H. Luo, X. Su, M.-T. Zhang, X. Lin, J. Sun, L. Wang, L. Deng, W. Zhang and R. Cao, Angew. Chem., Int. Ed., 2015, 54, 4870-4875.

23 A. Fujishima and K. Honda, Nature, 1972, 238, 37-38.

24 J. Wang, W. Cui, Q. Liu, Z. Xing, A. M. Asiri and X. Sun, Adv. Mater., 2016, 28, 215-230.

25 S. Zhang, S. E. Saji, Z. Yin, H. Zhang, Y. Du and C.-H. Yan, Adv. Mater., 2021, 33, 2005988.

26 J. Wang, X. Yue, Y. Yang, S. Sirisomboonchai, P. Wang, X. Ma, A. Abudula and G. Guan, J. Alloys Compd., 2020, 819, 153346. 
27 Y. Ji, L. Yang, X. Ren, G. Cui, X. Xiong and X. Sun, ACS Sustainable Chem. Eng., 2018, 6, 9555-9559.

28 B. M. Hunter, H. B. Gray and A. M. Müller, Chem. Rev., 2016, 116, 14120-14136.

29 J. Zhang, H. Zhou, X. Liu, J. Zhang, T. Peng, J. Yang, Y. Huang and S. Mu, J. Mater. Chem. A, 2016, 4, 15870-15879.

30 C. G. Morales-Guio, L.-A. Stern and X. Hu, Chem. Soc. Rev., 2014, 43, 6555-6569.

31 X. Zou and Y. Zhang, Chem. Soc. Rev., 2015, 44, 5148-5180.

32 S. Trasatti, J. Electroanal. Chem. Int. Electrochem., 1972, 39, 163-184.

33 S. Anantharaj, S. R. Ede, K. Sakthikumar, K. Karthick, S. Mishra and S. Kundu, ACS Catal., 2016, 6, 8069-8097.

34 Y. Shi and B. Zhang, Chem. Soc. Rev., 2016, 45, 1529-1541.

35 X. Chang, T. Wang, Z.-J. Zhao, P. Yang, J. Greeley, R. Mu, G. Zhang, Z. Gong, Z. Luo, J. Chen, Y. Cui, G. A. Ozin and J. Gong, Angew. Chem., Int. Ed., 2018, 57, 15415-15419.

36 T. Shinagawa, A. T. Garcia-Esparza and K. Takanabe, Sci. Rep., 2015, 5, 13801.

37 Y. Yan, B. Y. Xia, B. Zhao and X. Wang, J. Mater. Chem. A, 2016, 4, 17587-17603.

38 N. T. Suen, S. F. Hung, Q. Quan, N. Zhang, Y. J. Xu and H. M. Chen, Chem. Soc. Rev., 2017, 46, 337-365.

39 J. Rossmeisl, Z. W. Qu, H. Zhu, G. J. Kroes and J. K. Nørskov, J. Electroanal. Chem., 2007, 607, 83-89.

40 I. C. Man, H.-Y. Su, F. Calle-Vallejo, H. A. Hansen, J. I. Martínez, N. G. Inoglu, J. Kitchin, T. F. Jaramillo, J. K. Nørskov and J. Rossmeisl, ChemCatChem, 2011, 3, 1159-1165.

41 R. L. Doyle and M. E. G. Lyons, Photoelectrochemical Solar Fuel Production: From Basic Principles to Advanced Devices, Springer International Publishing, Cham, 2016, pp. 41-104.

42 Y. Matsumoto and E. Sato, Mater. Chem. Phys., 1986, 14, 397-426.

43 J. Tymoczko, V. Colic, A. Ganassin, W. Schuhmann and A. S. Bandarenka, Catal. Today, 2015, 244, 96-102.

44 X. Liu and L. Dai, Nat. Rev. Mater., 2016, 1, 16064.

45 R. Paul, L. Zhu, H. Chen, J. Qu and L. Dai, Adv. Mater., 2019, 31, 1806403.

46 H. Wang, H.-W. Lee, Y. Deng, Z. Lu, P.-C. Hsu, Y. Liu, D. Lin and Y. Cui, Nat. Commun., 2015, 6, 7261.

47 K. Liu, F. Wang, P. He, T. A. Shifa, Z. Wang, Z. Cheng, X. Zhan and J. He, Adv. Energy Mater., 2018, 8, 1703290.

48 Y.-N. Zhu, C.-Y. Cao, W.-J. Jiang, S.-L. Yang, J.-S. Hu, W.G. Song and L.-J. Wan, J. Mater. Chem. A, 2016, 4, 18470-18477.

49 X. Miao, D. Qu, D. Yang, B. Nie, Y. Zhao, H. Fan and Z. Sun, Adv. Mater., 2018, 30, 1704740.

50 J. Mao, J. Iocozzia, J. Huang, K. Meng, Y. Lai and Z. Lin, Energy Environ. Sci., 2018, 11, 772-799.

51 L. Li, P. Wang, Q. Shao and X. Huang, Chem. Soc. Rev., 2020, 49, 3072-3106.

52 Z. Zhou, Z. Pei, L. Wei, S. Zhao, X. Jian and Y. Chen, Energy Environ. Sci., 2020, 13, 3185-3206.

53 B. Kim, T. Kim, K. Lee and J. Li, ChemElectroChem, 2020, 7, 3578-3589.
54 S. Anantharaj and V. Aravindan, Adv. Energy Mater., 2020, 10, 1902666.

55 R. Wu, B. Xiao, Q. Gao, Y. R. Zheng, X. S. Zheng, J. F. Zhu, M. R. Gao and S. H. Yu, Angew. Chem., Int. Ed. Engl., 2018, 57, 15445-15449.

56 J. Yu, Y. Dai, Q. He, D. Zhao, Z. Shao and M. Ni, Mater. Rep.: Energy, 2021, 1, 100024.

57 J. Tian, Q. Liu, A. M. Asiri and X. Sun, J. Am. Chem. Soc., 2014, 136, 7587-7590.

58 Y. Lee, J. Suntivich, K. J. May, E. E. Perry and Y. Shao-Horn, J. Phys. Chem. Lett., 2012, 3, 399-404.

59 Y. Xu and B. Zhang, Chem. Soc. Rev., 2014, 43, 2439-2450.

60 Y. Li, Y. Sun, Y. Qin, W. Zhang, L. Wang, M. Luo, H. Yang and S. Guo, Adv. Energy Mater., 2020, 10, 1903120.

61 J. Feng, F. Lv, W. Zhang, P. Li, K. Wang, C. Yang, B. Wang, Y. Yang, J. Zhou, F. Lin, G.-C. Wang and S. Guo, Adv. Mater., 2017, 29, 1703798.

62 J. Park, S. Choi, A. Oh, H. Jin, J. Joo, H. Baik and K. Lee, Nanoscale Horiz., 2019, 4, 727-734.

63 M. Jahan, Z. Liu and K. P. Loh, Adv. Funct. Mater., 2013, 23, 5363-5372.

64 Z. Xing, Q. Liu, A. M. Asiri and X. Sun, Adv. Mater., 2014, 26, 5702-5707.

65 M. S. Xie, B. Y. Xia, Y. Li, Y. Yan, Y. Yang, Q. Sun, S. H. Chan, A. Fisher and X. Wang, Energy Environ. Sci., 2016, 9, 1687-1695.

66 Y. Zhu, H.-C. Chen, C.-S. Hsu, T.-S. Lin, C.-J. Chang, S.C. Chang, L.-D. Tsai and H. M. Chen, ACS Energy Lett., 2019, 4, 987-994.

67 A. Lasia and A. Rami, J. Electroanal. Chem. Interfacial Electrochem., 1990, 294, 123-141.

68 Y. Liang, X. Sun, A. M. Asiri and Y. He, Nanotechnology, 2016, 27, 12LT01.

69 B. C. M. Martindale and E. Reisner, Adv. Energy Mater., 2016, 6, 1502095.

70 C. Tang, N. Cheng, Z. Pu, W. Xing and X. Sun, Angew. Chem., Int. Ed., 2015, 54, 9351-9355.

71 C. Zhao, X. Dai, T. Yao, W. Chen, X. Wang, J. Wang, J. Yang, S. Wei, Y. Wu and Y. Li, J. Am. Chem. Soc., 2017, 139, 8078-8081.

72 N. Yang, C. Tang, K. Wang, G. Du, A. M. Asiri and X. Sun, Nano Res., 2016, 9, 3346-3354.

73 L. Yang, H. Qi, C. Zhang and X. Sun, Nanotechnology, 2016, 27, 23LT01.

74 T. Liu, L. Xie, J. Yang, R. Kong, G. Du, A. M. Asiri, X. Sun and L. Chen, ChemElectroChem, 2017, 4, 1840-1845.

75 W. Lu, T. Liu, L. Xie, C. Tang, D. Liu, S. Hao, F. Qu, G. Du, Y. Ma, A. M. Asiri and X. Sun, Small, 2017, 13, 1700805.

76 T. Liu, Y. Liang, Q. Liu, X. Sun, Y. He and A. M. Asiri, Electrochem. Commun., 2015, 60, 92-96.

77 P. Jiang, Q. Liu, Y. Liang, J. Tian, A. M. Asiri and X. Sun, Angew. Chem., Int. Ed., 2014, 53, 12855-12859.

78 F. Gloaguen, J. D. Lawrence and T. B. Rauchfuss, J. Am. Chem. Soc., 2001, 123, 9476-9477.

79 S.-K. Lee, S. D. George, W. E. Antholine, B. Hedman, K. O. Hodgson and E. I. Solomon, J. Am. Chem. Soc., 2002, 124, 6180-6193. 
80 D. Gao, R. Liu, J. Biskupek, U. Kaiser, Y.-F. Song and C. Streb, Angew. Chem., Int. Ed., 2019, 58, 4644-4648.

81 S. Chao, Z. Bai, Q. Cui, H. Yan, K. Wang and L. Yang, Carbon, 2015, 82, 77-86.

82 X. Zou, X. Huang, A. Goswami, R. Silva, B. R. Sathe, E. Mikmeková and T. Asefa, Angew. Chem., Int. Ed., 2014, 53, 4372-4376.

83 X. Lu and C. Zhao, Nat. Commun., 2015, 6, 6616.

84 R. D. L. Smith, M. S. Prévot, R. D. Fagan, S. Trudel and C. P. Berlinguette, J. Am. Chem. Soc., 2013, 135, 11580-11586.

85 C. Tang, A. M. Asiri, Y. Luo and X. Sun, ChemNanoMat, 2015, 1, 558-561.

86 C. Tang, Z. Pu, Q. Liu, A. M. Asiri and X. Sun, Electrochim. Acta, 2015, 153, 508-514.

87 L.-L. Feng, G. Yu, Y. Wu, G.-D. Li, H. Li, Y. Sun, T. Asefa, W. Chen and X. Zou, J. Am. Chem. Soc., 2015, 137, 14023-14026.

88 W. Zhou, X.-J. Wu, X. Cao, X. Huang, C. Tan, J. Tian, H. Liu, J. Wang and H. Zhang, Energy Environ. Sci., 2013, 6, 2921-2924.

89 Z. Pu, Y. Luo, A. M. Asiri and X. Sun, ACS Appl. Mater. Interfaces, 2016, 8, 4718-4723.

90 J. Shi, J. Hu, Y. Luo, X. Sun and A. M. Asiri, Catal. Sci. Technol., 2015, 5, 4954-4958.

91 C. Wang, M. Zhu, Z. Cao, P. Zhu, Y. Cao, X. Xu, C. Xu and Z. Yin, Appl. Catal., B, 2021, 291, 120071.

92 Z. Fang, L. Peng, H. Lv, Y. Zhu, C. Yan, S. Wang, P. Kalyani, $\mathrm{X}$. Wu and G. Yu, ACS Nano, 2017, 11, 9550-9557.

93 J. Yang, C. Lei, H. Wang, B. Yang, Z. Li, M. Qiu, X. Zhuang, C. Yuan, L. Lei, Y. Hou and X. Feng, Nanoscale, 2019, 11, 17571-17578.

94 C. Wei, C. Liu, L. Gao, Y. Sun, Q. Liu, X. Zhang and J. Guo, J. Alloys Compd., 2019, 796, 86-92.

95 W. Fang, D. Liu, Q. Lu, X. Sun and A. M. Asiri, Electrochem. Commun., 2016, 63, 60-64.

96 D. Kong, J. J. Cha, H. Wang, H. R. Lee and Y. Cui, Energy Environ. Sci., 2013, 6, 3553-3558.

97 M. A. Lukowski, A. S. Daniel, F. Meng, A. Forticaux, L. Li and S. Jin, J. Am. Chem. Soc., 2013, 135, 10274-10277.

98 T. Wang, J. Zhuo, K. Du, B. Chen, Z. Zhu, Y. Shao and M. Li, Adv. Mater., 2014, 26, 3761-3766.

99 D. Kong, H. Wang, J. J. Cha, M. Pasta, K. J. Koski, J. Yao and Y. Cui, Nano Lett., 2013, 13, 1341-1347.

100 W. F. Chen, C. H. Wang, K. Sasaki, N. Marinkovic, W. Xu, J. T. Muckerman, Y. Zhu and R. R. Adzic, Energy Environ. Sci., 2013, 6, 943-951.

101 T. A. Shifa, F. Wang, K. Liu, K. Xu, Z. Wang, X. Zhan, C. Jiang and J. He, Small, 2016, 12, 3802-3809.

102 Y. Sun, C. Liu, D. C. Grauer, J. Yano, J. R. Long, P. Yang and C. J. Chang, J. Am. Chem. Soc., 2013, 135, 17699-17702.

103 A. Ambrosi, C. K. Chua, A. Bonanni and M. Pumera, Chem. Rev., 2014, 114, 7150-7188.

104 S. Peng, L. Li, X. Han, W. Sun, M. Srinivasan, S. G. Mhaisalkar, F. Cheng, Q. Yan, J. Chen and S. Ramakrishna, Angew. Chem., Int. Ed., 2014, 53, 12594-12599.
105 C. Zequine, S. Bhoyate, F. Wang, X. Li, K. Siam, P. K. Kahol and R. K. Gupta, J. Alloys Compd., 2019, 784, 1-7.

106 Z. Peng, D. Jia, A. M. Al-Enizi, A. A. Elzatahry and G. Zheng, Adv. Energy Mater., 2015, 5, 1402031.

107 D. Kong, H. Wang, Z. Lu and Y. Cui, J. Am. Chem. Soc., 2014, 136, 4897-4900.

108 Q. Liu, J. Shi, J. Hu, A. M. Asiri, Y. Luo and X. Sun, ACS Appl. Mater. Interfaces, 2015, 7, 3877-3881.

109 Y.-F. Xu, M.-R. Gao, Y.-R. Zheng, J. Jiang and S.-H. Yu, Angew. Chem., Int. Ed., 2013, 52, 8546-8550.

110 M.-R. Gao, J.-X. Liang, Y.-R. Zheng, Y.-F. Xu, J. Jiang, Q. Gao, J. Li and S.-H. Yu, Nat. Commun., 2015, 6, 5982.

111 M.-R. Gao, Y.-F. Xu, J. Jiang, Y.-R. Zheng and S.-H. Yu, J. Am. Chem. Soc., 2012, 134, 2930-2933.

112 M.-R. Gao, X. Cao, Q. Gao, Y.-F. Xu, Y.-R. Zheng, J. Jiang and S.-H. Yu, ACS Nano, 2014, 8, 3970-3978.

113 H. Liang, F. Meng, M. Cabán-Acevedo, L. Li, A. Forticaux, L. Xiu, Z. Wang and S. Jin, Nano Lett., 2015, 15, 1421-1427.

114 D. Liu, Q. Lu, Y. Luo, X. Sun and A. M. Asiri, Nanoscale, 2015, 7, 15122-15126.

115 J. Zhang, T. Wang, D. Pohl, B. Rellinghaus, R. Dong, S. Liu, X. Zhuang and X. Feng, Angew. Chem., Int. Ed., 2016, 55, 6702-6707.

116 F. Du, L. Shi, Y. Zhang, T. Li, J. Wang, G. Wen, A. Alsaedi, T. Hayat, Y. Zhou and Z. Zou, Appl. Catal., B, 2019, 253, 246-252.

117 Y. V. Kaneti, Y. Guo, N. L. W. Septiani, M. Iqbal, X. Jiang, T. Takei, B. Yuliarto, Z. A. Alothman, D. Golberg and Y. Yamauchi, Chem. Eng. J., 2021, 405, 126580.

118 N. L. W. Septiani, Y. V. Kaneti, K. B. Fathoni, K. Kani, A. E. Allah, B. Yuliarto, Nugraha, H. K. Dipojono, Z. A. Alothman, D. Golberg and Y. Yamauchi, Chem. Mater., 2020, 32, 7005-7018.

119 P. Bhanja, Y. Kim, B. Paul, J. Lin, S. M. Alshehri, T. Ahamad, Y. V. Kaneti, A. Bhaumik and Y. Yamauchi, ChemCatChem, 2020, 12, 2091-2096.

120 N. L. W. Septiani, Y. V. Kaneti, K. B. Fathoni, Y. Guo, Y. Ide, B. Yuliarto, X. Jiang, Nugraha, H. K. Dipojono, D. Golberg and Y. Yamauchi, J. Mater. Chem. A, 2020, 8, 3035-3047.

121 M. W. Kanan and D. G. Nocera, Science, 2008, 321, 1072.

122 M. W. Kanan, Y. Surendranath and D. G. Nocera, Chem. Soc. Rev., 2009, 38, 109-114.

123 S. Cobo, J. Heidkamp, P.-A. Jacques, J. Fize, V. Fourmond, L. Guetaz, B. Jousselme, V. Ivanova, H. Dau, S. Palacin, M. Fontecave and V. Artero, Nat. Mater., 2012, 11, 802-807.

124 L. Song, S. Zhang, X. Wu and K. Wang, Vacuum, 2015, 112, 12-16.

125 J. A. Cecilia, A. Infantes-Molina, E. Rodríguez-Castellón and A. Jiménez-López, Appl. Catal., B, 2009, 92, 100-113.

126 W. Li, D. Xiong, X. Gao and L. Liu, Chem. Commun., 2019, 55, 8744-8763.

127 A. Dutta and N. Pradhan, J. Phys. Chem. Lett., 2017, 8, 144-152.

128 J. Xu, J. Li, D. Xiong, B. Zhang, Y. Liu, K.-H. Wu, I. Amorim, W. Li and L. Liu, Chem. Sci., 2018, 9, 3470-3476. 
129 E. J. Popczun, C. G. Read, C. W. Roske, N. S. Lewis and R. E. Schaak, Angew. Chem., Int. Ed., 2014, 53, 5427-5430.

130 Z. Huang, Z. Chen, Z. Chen, C. Lv, M. G. Humphrey and C. Zhang, Nano Energy, 2014, 9, 373-382.

131 J. F. Callejas, C. G. Read, E. J. Popczun, J. M. McEnaney and R. E. Schaak, Chem. Mater., 2015, 27, 3769-3774.

132 Z. Huang, Z. Chen, Z. Chen, C. Lv, H. Meng and C. Zhang, ACS Nano, 2014, 8, 8121-8129.

133 L. Feng, H. Vrubel, M. Bensimon and X. Hu, Phys. Chem. Chem. Phys., 2014, 16, 5917-5921.

134 T. Liu, D. Liu, F. Qu, D. Wang, L. Zhang, R. Ge, S. Hao, Y. Ma, G. Du, A. M. Asiri, L. Chen and X. Sun, Adv. Energy Mater., 2017, 7, 1700020.

135 Z. D. Wei, A. Z. Yan, Y. C. Feng, L. Li, C. X. Sun, Z. G. Shao and P. K. Shen, Electrochem. Commun., 2007, 9, 2709-2715.

136 L.-A. Stern, L. Feng, F. Song and X. Hu, Energy Environ. Sci., 2015, 8, 2347-2351.

137 C. Tang, R. Zhang, W. Lu, Z. Wang, D. Liu, S. Hao, G. Du, A. M. Asiri and X. Sun, Angew. Chem., Int. Ed., 2017, 56, 842-846.

138 M. Ledendecker, S. Krick Calderón, C. Papp, H.-P. Steinrück, M. Antonietti and M. Shalom, Angew. Chem., Int. Ed., 2015, 54, 12361-12365.

139 G.-F. Chen, T. Y. Ma, Z.-Q. Liu, N. Li, Y.-Z. Su, K. Davey and S.-Z. Qiao, Adv. Funct. Mater., 2016, 26, 3314-3323.

140 Q. Liu, J. Tian, W. Cui, P. Jiang, N. Cheng, A. M. Asiri and X. Sun, Angew. Chem., Int. Ed., 2014, 53, 6710-6714.

141 Y. Yan, B. Y. Xia, X. Ge, Z. Liu, A. Fisher and X. Wang, Chem. - Eur. J., 2015, 21, 18062-18067.

142 C. G. Read, J. F. Callejas, C. F. Holder and R. E. Schaak, ACS Appl. Mater. Interfaces, 2016, 8, 12798-12803.

143 T. A. Shifa, K. Yusupov, G. Solomon, A. Gradone, R. Mazzaro, E. Cattaruzza and A. Vomiero, ACS Catal., 2021, 11, 4520-4529.

144 D. J. Ham and J. S. Lee, Energies, 2009, 2, 873-899.

145 W.-F. Chen, J. T. Muckerman and E. Fujita, Chem. Commun., 2013, 49, 8896-8909.

146 P. Chen, K. Xu, Z. Fang, Y. Tong, J. Wu, X. Lu, X. Peng, H. Ding, C. Wu and Y. Xie, Angew. Chem., Int. Ed., 2015, 54, 14710-14714.

147 K. Xu, P. Chen, X. Li, Y. Tong, H. Ding, X. Wu, W. Chu, Z. Peng, C. Wu and Y. Xie, J. Am. Chem. Soc., 2015, 137, 4119-4125.

148 Q. Zhang, Y. Wang, Y. Wang, A. M. Al-Enizi, A. A. Elzatahry and G. Zheng, J. Mater. Chem. A, 2016, 4, 5713-5718.

149 B. Cao, G. M. Veith, J. C. Neuefeind, R. R. Adzic and P. G. Khalifah, J. Am. Chem. Soc., 2013, 135, 19186-19192.

150 W.-F. Chen, K. Sasaki, C. Ma, A. I. Frenkel, N. Marinkovic, J. T. Muckerman, Y. Zhu and R. R. Adzic, Angew. Chem., Int. Ed., 2012, 51, 6131-6135.

151 X. Jia, Y. Zhao, G. Chen, L. Shang, R. Shi, X. Kang, G. I. N. Waterhouse, L.-Z. Wu, C.-H. Tung and T. Zhang, Adv. Energy Mater., 2016, 6, 1502585.

152 M. C. Weidman, D. V. Esposito, Y.-C. Hsu and J. G. Chen, J. Power Sources, 2012, 202, 11-17.

153 E. C. Weigert, D. V. Esposito and J. G. Chen, J. Power Sources, 2009, 193, 501-506.
154 L. Liao, S. Wang, J. Xiao, X. Bian, Y. Zhang, M. D. Scanlon, X. Hu, Y. Tang, B. Liu and H. H. Girault, Energy Environ. Sci., 2014, 7, 387-392.

155 H. Vrubel and X. Hu, Angew. Chem., Int. Ed., 2012, 51, 12703-12706.

156 B. Wang, X. Wu, X. Zhang, G. Pang and S. Li, New J. Chem., 2020, 44, 1147-1156.

157 J.-S. Li, L.-X. Kong, Z. Wu, S. Zhang, X.-Y. Yang, J.-Q. Sha and G.-D. Liu, Carbon, 2019, 145, 694-700.

158 Z.-Y. Wu, W.-B. Ji, B.-C. Hu, H.-W. Liang, X.-X. Xu, Z.-L. Yu, B.-Y. Li and S.-H. Yu, Nano Energy, 2018, 51, 286-293.

159 C. Tang and S.-Z. Qiao, Matter, 2019, 1, 1454-1455.

160 J. Zhang, L. Qu, G. Shi, J. Liu, J. Chen and L. Dai, Angew. Chem., Int. Ed., 2016, 55, 2230-2234.

161 Y. Zheng, Y. Jiao, Y. Zhu, L. H. Li, Y. Han, Y. Chen, A. Du, M. Jaroniec and S. Z. Qiao, Nat. Commun., 2014, 5, 3783.

162 R. Wu, J. Zhang, Y. Shi, D. Liu and B. Zhang, J. Am. Chem. Soc., 2015, 137, 6983-6986.

163 J. Lai, S. Li, F. Wu, M. Saqib, R. Luque and G. Xu, Energy Environ. Sci., 2016, 9, 1210-1214.

164 S. Chen, J. Duan, M. Jaroniec and S. Z. Qiao, Angew. Chem., Int. Ed., 2013, 52, 13567-13570.

165 X. Sun, F. Liu, X. Chen, C. Li, J. Yu and M. Pan, Electrochim. Acta, 2019, 307, 206-213.

166 Y. Sun, B. Huang, Y. Li, Y. Xing, M. Luo, N. Li, Z. Xia, Y. Qin, D. Su, L. Wang and S. Guo, Chem. Mater., 2019, 31, 8136-8144.

167 J. Ding, Q. Shao, Y. Feng and X. Huang, Nano Energy, 2018, 47, 1-7.

168 P. W. Menezes, C. Panda, S. Garai, C. Walter, A. Guiet and M. Driess, Angew. Chem., Int. Ed., 2018, 57, 15237-15242.

169 X. Wang, F. Li, W. Li, W. Gao, Y. Tang and R. Li, J. Mater. Chem. A, 2017, 5, 17982-17989.

170 Y. Liu, S. Jiang, S. Li, L. Zhou, Z. Li, J. Li and M. Shao, Appl. Catal., B, 2019, 247, 107-114.

171 Y. Guo, X. Zhou, J. Tang, S. Tanaka, Y. V. Kaneti, J. Na, B. Jiang, Y. Yamauchi, Y. Bando and Y. Sugahara, Nano Energy, 2020, 75, 104913.

172 Y. Gong, Z. Yang, Y. Lin, T. Zhou, J. Li, F. Jiao and W. Wang, Dalton Trans., 2019, 48, 7025.

173 F. Li, R. Xu, Y. Li, F. Liang, D. Zhang, W.-F. Fu and X.-J. Lv, Carbon, 2019, 145, 521-528.

174 X. Wang, W. Li, D. Xiong and L. Liu, J. Mater. Chem. A, 2016, 4, 5639-5646.

175 A. Chunduri, S. Gupta, O. Bapat, A. Bhide, R. Fernandes, M. K. Patel, V. Bambole, A. Miotello and N. Patel, Appl. Catal., B, 2019, 259, 118051.

176 P. W. Menezes, A. Indra, I. Zaharieva, C. Walter, S. Loos, S. Hoffmann, R. Schlögl, H. Dau and M. Driess, Energy Environ. Sci., 2019, 12, 988-999.

177 R. Zhang, J. Huang, G. Chen, W. Chen, C. Song, C. Li and K. Ostrikov, Appl. Catal., B, 2019, 254, 414-423.

178 A. Wu, Y. Xie, H. Ma, C. Tian, Y. Gu, H. Yan, X. Zhang, G. Yang and H. Fu, Nano Energy, 2018, 44, 353-363.

179 J. Xing, Y. Li, S. Guo, T. Jin, H. Li, Y. Wang and L. Jiao, Electrochim. Acta, 2019, 298, 305-312. 
180 M. A. R. Anjum, M. H. Lee and J. S. Lee, ACS Catal., 2018, 8, 8296-8305.

181 L. Hui, Y. Xue, B. Huang, H. Yu, C. Zhang, D. Zhang, D. Jia, Y. Zhao, Y. Li, H. Liu and Y. Li, Nat. Commun., 2018, 9, 5309.

182 R. Matsuoka, R. Sakamoto, K. Hoshiko, S. Sasaki, H. Masunaga, K. Nagashio and H. Nishihara, J. Am. Chem. Soc., 2017, 139, 3145-3152.

183 G. Li, Y. Li, H. Liu, Y. Guo, Y. Li and D. Zhu, Chem. Commun., 2010, 46, 3256-3258.

184 H.-Y. Si, Q.-X. Deng, L.-C. Chen, L. Wang, X.-Y. Liu, W.-S. Wu, Y.-H. Zhang, J.-M. Zhou and H.-L. Zhang, J. Alloys Compd., 2019, 794, 261-267.

185 R. F. Service, Science, 2014, 345, 610.

186 Y. Liu, Y. Su, X. Quan, X. Fan, S. Chen, H. Yu, H. Zhao, Y. Zhang and J. Zhao, ACS Catal., 2018, 8, 1186-1191.

187 I. Dybkjaer, Ammonia, catalysis and manufacture ed. A. Nielsen, Springer, Heidelberg, 1995, pp. 199-308.

188 R. Zhao, H. Xie, L. Chang, X. Zhang, X. Zhu, X. Tong, T. Wang, Y. Luo, P. Wei, Z. Wang and X. Sun, EnergyChem, 2019, 1, 100011.

189 C. J. M. van der Ham, M. T. M. Koper and D. G. H. Hetterscheid, Chem. Soc. Rev., 2014, 43, 5183-5191.

190 H. J. M. van Grinsven, L. Bouwman, K. G. Cassman, H. M. van Es, M. L. McCrackin and A. H. W. Beusen, J. Environ. Qual., 2015, 44, 356-367.

191 R. Qin, P. Liu, G. Fu and N. Zheng, Small Methods, 2018, 2, 1700286.

192 Q. Li, L. He, C. Sun and X. Zhang, J. Phys. Chem. C, 2017, 121, 27563-27568.

193 J. Zhao and Z. Chen, J. Am. Chem. Soc., 2017, 139, 12480-12487.

194 B. H. R. Suryanto, H.-L. Du, D. Wang, J. Chen, A. N. Simonov and D. R. MacFarlane, Nat. Catal., 2019, 2, 290-296.

195 D. Wang, L. M. Azofra, M. Harb, L. Cavallo, X. Zhang, B. H. R. Suryanto and D. R. MacFarlane, ChemSusChem, 2018, 11, 3416-3422.

196 M. A. Shipman and M. D. Symes, Catal. Today, 2017, 286, 57-68.

197 X. Guo, H. Du, F. Qu and J. Li, J. Mater. Chem. A, 2019, 7, 3531-3543.

198 T. Spatzal, M. Aksoyoglu, L. Zhang, S. L. A. Andrade, E. Schleicher, S. Weber, D. C. Rees and O. Einsle, Science, 2011, 334, 940.

199 L. C. Seefeldt, B. M. Hoffman and D. R. Dean, Annu. Rev. Biochem., 2009, 78, 701-722.

200 K. Kugler, B. Ohs, M. Scholz and M. Wessling, Phys. Chem. Chem. Phys., 2014, 16, 6129-6138.

201 E. Skúlason, T. Bligaard, S. Gudmundsdóttir, F. Studt, J. Rossmeisl, F. Abild-Pedersen, T. Vegge, H. Jónsson and J. K. Nørskov, Phys. Chem. Chem. Phys., 2012, 14, 1235-1245.

202 P. Wang, F. Chang, W. Gao, J. Guo, G. Wu, T. He and P. Chen, Nat. Chem., 2017, 9, 64-70.

203 G. Ertl, Catal. Rev., 1980, 21, 201-223.

204 D. Senthil Raja, H.-W. Lin and S.-Y. Lu, Nano Energy, 2019, 57, 1-13.
205 Y. Abghoui, A. L. Garden, V. F. Hlynsson, S. Björgvinsdóttir, H. Ólafsdóttir and E. Skúlason, Phys. Chem. Chem. Phys., 2015, 17, 4909-4918.

206 Á. B. Höskuldsson, Y. Abghoui, A. B. Gunnarsdóttir and E. Skúlason, ACS Sustainable Chem. Eng., 2017, 5, 10327-10333.

207 L. Huang, J. Wu, P. Han, A. M. Al-Enizi, T. M. Almutairi, L. Zhang and G. Zheng, Small Methods, 2019, 3, 1800386.

208 M. Zhu, Q. Shao, Y. Qian and X. Huang, Nano Energy, 2019, 56, 330-337.

209 J. Han, Z. Liu, Y. Ma, G. Cui, F. Xie, F. Wang, Y. Wu, S. Gao, Y. Xu and X. Sun, Nano Energy, 2018, 52, 264-270.

210 W. Kong, Z. Liu, J. Han, L. Xia, Y. Wang, Q. Liu, X. Shi, Y. $\mathrm{Wu}, \mathrm{Y} . \mathrm{Xu}$ and X. Sun, Inorg. Chem. Front., 2019, 6, 423-427.

211 C. Ling, Y. Ouyang, Q. Li, X. Bai, X. Mao, A. Du and J. Wang, Small Methods, 2019, 3, 1800376.

212 Y. Abghoui, A. L. Garden, J. G. Howalt, T. Vegge and E. Skúlason, ACS Catal., 2016, 6, 635-646.

213 H.-L. Du, T. R. Gengenbach, R. Hodgetts, D. R. MacFarlane and A. N. Simonov, ACS Sustainable Chem. Eng., 2019, 7, 6839-6850.

214 C. J. H. Jacobsen, Chem. Commun., 2000, 1057-1058.

215 C. D. Zeinalipour-Yazdi, J. S. J. Hargreaves and C. R. A. Catlow, J. Phys. Chem. C, 2015, 119, 28368-28376.

216 L. F. Greenlee, J. N. Renner and S. L. Foster, ACS Catal., 2018, 8, 7820-7827.

217 S. E. Saji, H. Lu, Z. Lu, A. Carroll and Z. Yin, Small Methods, 2021, 5, 2000694.

218 W. Guo, K. Zhang, Z. Liang, R. Zou and Q. Xu, Chem. Soc. Rev., 2019, 48, 5658-5716.

219 C. Tang and S.-Z. Qiao, Joule, 2019, 3, 1573-1575.

220 S. Z. Andersen, V. Čolić, S. Yang, J. A. Schwalbe, A. C. Nielander, J. M. McEnaney, K. Enemark-Rasmussen, J. G. Baker, A. R. Singh, B. A. Rohr, M. J. Statt, S. J. Blair, S. Mezzavilla, J. Kibsgaard, P. C. K. Vesborg, M. Cargnello, S. F. Bent, T. F. Jaramillo, I. E. L. Stephens, J. K. Nørskov and I. Chorkendorff, Nature, 2019, 570, 504-508.

221 S. Z. Andersen, M. J. Statt, V. J. Bukas, S. G. Shapel, J. B. Pedersen, K. Krempl, M. Saccoccio, D. Chakraborty, J. Kibsgaard, P. C. K. Vesborg, J. Nørskov and I. Chorkendorff, Energy Environ. Sci., 2020, 13, 4291-4300.

222 M. Shao, Y. Shao, W. Chen, K. L. Ao, R. Tong, Q. Zhu, I. N. Chan, W. F. Ip, X. Shi and H. Pan, Phys. Chem. Chem. Phys., 2018, 20, 14504-14512.

223 D. Bao, Q. Zhang, F.-L. Meng, H.-X. Zhong, M.-M. Shi, Y. Zhang, J.-M. Yan, Q. Jiang and X.-B. Zhang, Adv. Mater., 2017, 29, 1604799.

224 J. H. Montoya, C. Tsai, A. Vojvodic and J. K. Nørskov, ChemSusChem, 2015, 8, 2180-2186.

225 Á. Logadóttir and J. K. Nørskov, J. Catal., 2003, 220, 273-279.

226 H. Wang, H. Yu, Z. Wang, Y. Li, Y. Xu, X. Li, H. Xue and L. Wang, Small, 2019, 15, 1804769.

227 M. Nazemi and M. A. El-Sayed, J. Phys. Chem. Lett., 2018, 9, 5160-5166. 
228 J. Wang, L. Yu, L. Hu, G. Chen, H. Xin and X. Feng, Nat. Commun., 2018, 9, 1795.

229 M.-M. Shi, D. Bao, S.-J. Li, B.-R. Wulan, J.-M. Yan and Q. Jiang, Adv. Energy Mater., 2018, 8, 1800124.

230 Z. Geng, Y. Liu, X. Kong, P. Li, K. Li, Z. Liu, J. Du, M. Shu, R. Si and J. Zeng, Adv. Mater., 2018, 30, 1870301.

231 N. Zhang, L. Li, J. Wang, Z. Hu, Q. Shao, X. Xiao and X. Huang, Angew. Chem., 2020, 59, 8066-8071.

232 H. Huang, L. Xia, X. Shi, A. M. Asiri and X. Sun, Chem. Commun., 2018, 54, 11427-11430.

233 X. Li, H. Xie and J. Mao, J. Mater. Sci., 2020, 55, 5203-5210.

234 F. Akagi, T. Matsuo and H. Kawaguchi, Angew. Chem., Int. Ed., 2007, 46, 8778-8781.

235 T. Shima, S. Hu, G. Luo, X. Kang, Y. Luo and Z. Hou, Science, 2013, 340, 1549.

236 Y. Kobayashi, Y. Tang, T. Kageyama, H. Yamashita, N. Masuda, S. Hosokawa and H. Kageyama, J. Am. Chem. Soc., 2017, 139, 18240-18246.

237 C. J. H. Jacobsen, S. Dahl, B. S. Clausen, S. Bahn, A. Logadottir and J. K. Nørskov, J. Am. Chem. Soc., 2001, 123, 8404-8405.

238 C. I. Ezeh, X. Yang, J. He, C. Snape and X. M. Cheng, Ultrason. Sonochem., 2018, 42, 48-56.

239 R. Schlögl, Angew. Chem., Int. Ed., 2003, 42, 2004-2008.

240 S. Back and Y. Jung, Phys. Chem. Chem. Phys., 2016, 18, 9161-9166.

241 W. Peng, M. Luo, X. Xu, K. Jiang, M. Peng, D. Chen, T. S. Chan and Y. Tan, Adv. Energy Mater., 2020, 10, 2001364.

242 J. Hou, M. Yang and J. Zhang, Nanoscale, 2020, 12, 6900-6920.

243 A. P. Leontiev, O. A. Brylev and K. S. Napolskii, Electrochim. Acta, 2015, 155, 466-473.

244 L. Ji, X. Shi, A. M. Asiri, B. Zheng and X. Sun, Inorg. Chem., 2018, 57, 14692-14697.

245 D. Yang, T. Chen and Z. Wang, J. Mater. Chem. A, 2017, 5, 18967-18971.

246 I. Matanović, F. H. Garzon and N. J. Henson, Phys. Chem. Chem. Phys., 2014, 16, 3014-3026.

247 D. Yao, C. Tang, L. Li, B. Xia, A. Vasileff, H. Jin, Y. Zhang and S. Z. Qiao, Adv. Energy Mater., 2020, 10, 2001289.

248 Z. Fang, P. Wu, Y. Qian and G. Yu, Angew. Chem., Int. Ed., 2021, 60, 4275-4281.

249 T. J. Del Castillo, N. B. Thompson and J. C. Peters, J. Am. Chem. Soc., 2016, 138, 5341-5350.

250 J. S. Anderson, J. Rittle and J. C. Peters, Nature, 2013, 501, 84-87.

251 T. M. Buscagan, P. H. Oyala and J. C. Peters, Angew. Chem., Int. Ed., 2017, 56, 6921-6926.

252 M.-T. Nguyen, N. Seriani and R. Gebauer, Phys. Chem. Chem. Phys., 2015, 17, 14317-14322.

253 S. Licht, B. Cui, B. Wang, F.-F. Li, J. Lau and S. Liu, Science, 2014, 345, 637.

254 F.-F. Li and S. Licht, Inorg. Chem., 2014, 53, $10042-10044$.

255 J. Kong, A. Lim, C. Yoon, J. H. Jang, H. C. Ham, J. Han, S. Nam, D. Kim, Y.-E. Sung, J. Choi and H. S. Park, ACS Sustainable Chem. Eng., 2017, 5, 10986-10995.
256 S. Chen, S. Perathoner, C. Ampelli, C. Mebrahtu, D. Su and G. Centi, Angew. Chem., Int. Ed., 2017, 56, 2699-2703.

257 S. Chen, S. Perathoner, C. Ampelli, C. Mebrahtu, D. Su and G. Centi, ACS Sustainable Chem. Eng., 2017, 5, 7393-7400.

258 F. Zhou, L. M. Azofra, M. Ali, M. Kar, A. N. Simonov, C. McDonnell-Worth, C. Sun, X. Zhang and D. R. MacFarlane, Energy Environ. Sci., 2017, 10, 2516-2520.

259 B. T. Hang and D. H. Thang, J. Alloys Compd., 2016, 655, 44-49.

260 X. Cui, C. Tang, X.-M. Liu, C. Wang, W. Ma and Q. Zhang, Chem. - Eur. J., 2018, 24, 18494-18501.

261 X. Zhu, Z. Liu, Q. Liu, Y. Luo, X. Shi, A. M. Asiri, Y. Wu and X. Sun, Chem. Commun., 2018, 54, 11332-11335.

262 X. Zhu, J. Zhao, L. Ji, T. Wu, T. Wang, S. Gao, A. A. Alshehri, K. A. Alzahrani, Y. Luo, Y. Xiang, B. Zheng and X. Sun, Nano Res., 2020, 13, 209-214.

263 K. Ogura, J. R. Ferrell, A. V. Cugini, E. S. Smotkin and M. D. Salazar-Villalpando, Electrochim. Acta, 2010, 56, 381-386.

264 X. Zhu, Z. Liu, H. Wang, R. Zhao, H. Chen, T. Wang, F. Wang, Y. Luo, Y. Wu and X. Sun, Chem. Commun., 2019, 55, 3987-3990.

265 X. Xiang, Z. Wang, X. Shi, M. Fan and X. Sun, ChemCatChem, 2018, 10, 4530-4535.

266 X. Tang, R. Jia, T. Zhai and H. Xia, ACS Appl. Mater. Interfaces, 2015, 7, 27518-27525.

267 L. Hu, A. Khaniya, J. Wang, G. Chen, W. E. Kaden and X. Feng, ACS Catal., 2018, 8, 9312-9319.

268 X. P. Wang, Y. Hu and J. Chen, Int. Arch. Photogramm. Remote Sens. Spatial Inf. Sci., 2018, XLII-3, 1795-1798.

269 X.-F. Li, Q.-K. Li, J. Cheng, L. Liu, Q. Yan, Y. Wu, X.-H. Zhang, Z.-Y. Wang, Q. Qiu and Y. Luo, J. Am. Chem. Soc., 2016, 138, 8706-8709.

270 J. Zhao, L. Zhang, X.-Y. Xie, X. Li, Y. Ma, Q. Liu, W.-H. Fang, X. Shi, G. Cui and X. Sun, J. Mater. Chem. A, 2018, 6, 24031-24035.

271 W. Li, Y. Bai, F. Li, C. Liu, K.-Y. Chan, X. Feng and X. Lu, J. Mater. Chem., 2012, 22, 4025-4031.

272 X. Zhang, Q. Liu, X. Shi, A. M. Asiri, Y. Luo, X. Sun and T. Li, J. Mater. Chem. A, 2018, 6, 17303-17306.

273 K. Jia, Y. Wang, Q. Pan, B. Zhong, Y. Luo, G. Cui, X. Guo and X. Sun, Nanoscale Adv., 2019, 1, 961-964.

274 Y. Wang, K. Jia, Q. Pan, Y. Xu, Q. Liu, G. Cui, X. Guo and X. Sun, ACS Sustainable Chem. Eng., 2019, 7, 117-122.

275 L. Zhang, X. Ji, X. Ren, Y. Ma, X. Shi, Z. Tian, A. M. Asiri, L. Chen, B. Tang and X. Sun, Adv. Mater., 2018, 30, 1800191.

276 F. Lai, W. Zong, G. He, Y. Xu, H. Huang, B. Weng, D. Rao, J. A. Martens, J. Hofkens, I. P. Parkin and T. Liu, Angew. Chem., Int. Ed., 2020, 59, 13320-13327.

277 H. Cheng, L.-X. Ding, G.-F. Chen, L. Zhang, J. Xue and H. Wang, Adv. Mater., 2018, 30, 1803694.

278 X. Yang, J. Nash, J. Anibal, M. Dunwell, S. Kattel, E. Stavitski, K. Attenkofer, J. G. Chen, Y. Yan and B. Xu, J. Am. Chem. Soc., 2018, 140, 13387-13391.

279 S. Zhang, W. Gong, Y. Lv, H. Wang, M. Han, G. Wang, T. Shi and H. Zhang, Chem. Commun., 2019, 55, 12376-12379. 
280 M. Yuan, H. Zhang, D. Gao, H. He, Y. Sun, P. Lu, S. Dipazir, Q. Li, L. Zhou, S. Li, Z. Liu, J. Yang, Y. Xie, H. Zhao and G. Zhang, J. Mater. Chem. A, 2020, 8, 2691-2700.

281 J. Shao, W. Sheng, M. Wang, S. Li, J. Chen, Y. Zhang and S. Cao, Appl. Catal., B, 2017, 209, 311-319.

282 I. A. Amar, R. Lan, J. Humphreys and S. Tao, Catal. Today, 2017, 286, 51-56.

283 R. Zhang, X. Ren, X. Shi, F. Xie, B. Zheng, X. Guo and X. Sun, ACS Appl. Mater. Interfaces, 2018, 10, 28251-28255.

284 L. Yang, T. Wu, R. Zhang, H. Zhou, L. Xia, X. Shi, H. Zheng, Y. Zhang and X. Sun, Nanoscale, 2019, 11, 1555-1562.

285 B. H. R. Suryanto, D. Wang, L. M. Azofra, M. Harb, L. Cavallo, R. Jalili, D. R. G. Mitchell, M. Chatti and D. R. MacFarlane, ACS Energy Lett., 2019, 4, 430-435.

286 Y. Abghoui, S. B. Sigtryggsson and E. Skúlason, ChemSusChem, 2019, 12, 4265-4273.

287 R. Michalsky, Y.-J. Zhang, A. J. Medford and A. A. Peterson, J. Phys. Chem. C, 2014, 118, 13026-13034.

288 X. Xu, F. Nosheen and X. Wang, Chem. Mater., 2016, 28, 6313-6320.

289 Y. Shi, Y. Yang, Y.-W. Li and H. Jiao, ACS Catal., 2016, 6, 6790-6803.

290 I. Matanovic and F. H. Garzon, Phys. Chem. Chem. Phys., 2018, 20, 14679-14687.

291 G. Gao, A. P. O'Mullane and A. Du, ACS Catal., 2017, 7, 494-500.

292 M. Naguib, M. Kurtoglu, V. Presser, J. Lu, J. Niu, M. Heon, L. Hultman, Y. Gogotsi and M. W. Barsoum, Adv. Mater., 2011, 23, 4248-4253.

293 L. M. Azofra, N. Li, D. R. MacFarlane and C. Sun, Energy Environ. Sci., 2016, 9, 2545-2549.

294 X. Ren, J. Zhao, Q. Wei, Y. Ma, H. Guo, Q. Liu, Y. Wang, G. Cui, A. M. Asiri, B. Li, B. Tang and X. Sun, ACS Cent. Sci., 2019, 5, 116-121.

295 Y. Abghoui and E. Skúlason, Catal. Today, 2017, 286, 69-77.

296 Y. Abghoui and E. Skúlason, J. Phys. Chem. C, 2017, 121, 6141-6151.

297 R. Zhang, Y. Zhang, X. Ren, G. Cui, A. M. Asiri, B. Zheng and X. Sun, ACS Sustainable Chem. Eng., 2018, 6, 9545-9549.

298 X. Zhang, R.-M. Kong, H. Du, L. Xia and F. Qu, Chem. Commun., 2018, 54, 5323-5325.

299 X. Zhu, T. Wu, L. Ji, Q. Liu, Y. Luo, G. Cui, Y. Xiang, Y. Zhang, B. Zheng and X. Sun, Chem. Commun., 2020, 56, 731-734.

300 H. Huang, L. Xia, R. Cao, Z. Niu, H. Chen, Q. Liu, T. Li, X. Shi, A. M. Asiri and X. Sun, Chem. - Eur. J., 2019, 25, 1914-1917.

301 Y. Song, D. Johnson, R. Peng, D. K. Hensley, P. V. Bonnesen, L. Liang, J. Huang, F. Yang, F. Zhang, R. Qiao, A. P. Baddorf, T. J. Tschaplinski, N. L. Engle, M. C. Hatzell, Z. Wu, D. A. Cullen, H. M. Meyer, B. G. Sumpter and A. J. Rondinone, Sci. Adv., 2018, 4, e1700336.

302 D. Yu, E. Nagelli, F. Du and L. Dai, J. Phys. Chem. Lett., 2010, 1, 2165-2173.
303 Y. Jiao, Y. Zheng, K. Davey and S.-Z. Qiao, Nat. Energy, 2016, 1, 16130.

304 W. Li, T. Wu, S. Zhang, Y. Liu, C. Zhao, G. Liu, G. Wang, H. Zhang and H. Zhao, Chem. Commun., 2018, 54, 11188-11191.

305 D. S. Su, S. Perathoner and G. Centi, Chem. Rev., 2013, 113, 5782-5816.

306 T. Wu, X. Li, X. Zhu, S. Mou, Y. Luo, X. Shi, A. M. Asiri, Y. Zhang, B. Zheng, H. Zhao and X. Sun, Chem. Commun., 2020, 56, 1831-1834.

307 F. Pan, H. Zhang, K. Liu, D. Cullen, K. More, M. Wang, Z. Feng, G. Wang, G. Wu and Y. Li, ACS Catal., 2018, 8, 3116-3122.

308 D. W. Stephan, Acc. Chem. Res., 2015, 48, 306-316.

309 C. Zhao, S. Zhang, M. Han, X. Zhang, Y. Liu, W. Li, C. Chen, G. Wang, H. Zhang and H. Zhao, ACS Energy Lett., 2019, 4, 377-383.

310 S. Mukherjee, D. A. Cullen, S. Karakalos, K. Liu, H. Zhang, S. Zhao, H. Xu, K. L. More, G. Wang and G. Wu, Nano Energy, 2018, 48, 217-226.

311 C. Lv, Y. Qian, C. Yan, Y. Ding, Y. Liu, G. Chen and G. Yu, Angew. Chem., Int. Ed., 2018, 57, 10246-10250.

312 G. Peng, J. Wu, M. Wang, J. Niklas, H. Zhou and C. Liu, Nano Lett., 2020, 20, 2879-2885.

313 X. Mao, S. Zhou, C. Yan, Z. Zhu and A. Du, Phys. Chem. Chem. Phys., 2019, 21, 1110-1116.

314 J. Zhao, X. Ren, X. Li, D. Fan, X. Sun, H. Ma, Q. Wei and D. Wu, Nanoscale, 2019, 11, 4231-4235.

315 Z. Geng, Y. Liu, X. Kong, P. Li, K. Li, Z. Liu, J. Du, M. Shu, R. Si and J. Zeng, Adv. Mater., 2018, 30, 1803498.

316 X. Yu, P. Han, Z. Wei, L. Huang, Z. Gu, S. Peng, J. Ma and G. Zheng, Joule, 2018, 2, 1610-1622.

317 F. Ma, Y. Jiao, G. Gao, Y. Gu, A. Bilic, Z. Chen and A. Du, Nano Lett., 2016, 16, 3022-3028.

318 C. Liu, Q. Li, C. Wu, J. Zhang, Y. Jin, D. R. MacFarlane and C. Sun, J. Am. Chem. Soc., 2019, 141, 2884-2888.

319 C. Hering-Junghans, Angew. Chem., Int. Ed., 2018, 57, 6738-6740.

320 B. M. Hoffman, D. Lukoyanov, Z.-Y. Yang, D. R. Dean and L. C. Seefeldt, Chem. Rev., 2014, 114, 4041-4062.

321 Y. Fu and A. Manthiram, RSC Adv., 2012, 2, 5927-5929.

322 H. Chen, X. Zhu, H. Huang, H. Wang, T. Wang, R. Zhao, H. Zheng, A. M. Asiri, Y. Luo and X. Sun, Chem. Commun., 2019, 55, 3152-3155.

323 L. Xia, J. Yang, H. Wang, R. Zhao, H. Chen, W. Fang, A. M. Asiri, F. Xie, G. Cui and X. Sun, Chem. Commun., 2019, 55, 3371-3374.

324 T. Wu, P. Li, H. Wang, R. Zhao, Q. Zhou, W. Kong, M. Liu, Y. Zhang, X. Sun and F. Gong, Chem. Commun., 2019, 55, 2684-2687.

325 C. Choi, S. Back, N.-Y. Kim, J. Lim, Y.-H. Kim and Y. Jung, ACS Catal., 2018, 8, 7517-7525.

326 C. Chen, D. Yan, Y. Wang, Y. Zhou, Y. Zou, Y. Li and S. Wang, Small, 2019, 15, 1805029.

327 X. Zhang, T. Wu, H. Wang, R. Zhao, H. Chen, T. Wang, P. Wei, Y. Luo, Y. Zhang and X. Sun, ACS Catal., 2019, 9, 4609-4615. 
328 W. Qiu, X.-Y. Xie, J. Qiu, W.-H. Fang, R. Liang, X. Ren, X. Ji, G. Cui, A. M. Asiri, G. Cui, B. Tang and X. Sun, Nat. Commun., 2018, 9, 3485.

329 Z. Wei, Y. Zhang, S. Wang, C. Wang and J. Ma, J. Mater. Chem. A, 2018, 6, 13790-13796.

330 L. Zhang, L.-X. Ding, G.-F. Chen, X. Yang and H. Wang, Angew. Chem., 2019, 131, 2638-2642.

331 X. Zhu, S. Mou, Q. Peng, Q. Liu, Y. Luo, G. Chen, S. Gao and X. Sun, J. Mater. Chem. A, 2020, 8, 1545-1556.

332 G.-F. Chen, X. Cao, S. Wu, X. Zeng, L.-X. Ding, M. Zhu and H. Wang, J. Am. Chem. Soc., 2017, 139, 9771-9774.

333 Y. Yang, S.-Q. Wang, H. Wen, T. Ye, J. Chen, C.-P. Li and M. Du, Angew. Chem., Int. Ed., 2019, 58, 15362-15366.

334 D. T. Whipple and P. J. A. Kenis, J. Phys. Chem. Lett., 2010, 1, 3451-3458.

335 J. Artz, T. E. Müller, K. Thenert, J. Kleinekorte, R. Meys, A. Sternberg, A. Bardow and W. Leitner, Chem. Rev., 2018, 118, 434-504.

336 A. M. Appel, J. E. Bercaw, A. B. Bocarsly, H. Dobbek, D. L. DuBois, M. Dupuis, J. G. Ferry, E. Fujita, R. Hille, P. J. A. Kenis, C. A. Kerfeld, R. H. Morris, C. H. F. Peden, A. R. Portis, S. W. Ragsdale, T. B. Rauchfuss, J. N. H. Reek, L. C. Seefeldt, R. K. Thauer and G. L. Waldrop, Chem. Rev., 2013, 113, 6621-6658.

337 S. Roy, A. Cherevotan and S. C. Peter, ACS Energy Lett., 2018, 3, 1938-1966.

338 J. Wu, Y. Huang, W. Ye and Y. Li, Adv. Sci., 2017, 4, 1700194. 339 P. Lu, X. Tan, H. Zhao, Q. Xiang, K. Liu, X. Zhao, X. Yin, X. Li, X. Hai, S. Xi, A. T. S. Wee, S. J. Pennycook, X. Yu, M. Yuan, J. Wu, G. Zhang, S. C. Smith and Z. Yin, ACS Nano, 2021, 15, 5671-5678.

340 R. Kortlever, J. Shen, K. J. P. Schouten, F. Calle-Vallejo and M. T. M. Koper, J. Phys. Chem. Lett., 2015, 6, 4073-4082.

341 L. R. L. Ting and B. S. Yeo, Curr. Opin. Electrochem., 2018, 8, 126-134.

342 L. Wang, W. Chen, D. Zhang, Y. Du, R. Amal, S. Qiao, J. Wu and Z. Yin, Chem. Soc. Rev., 2019, 48, 5310-5349.

343 R. Zhao, P. Ding, P. Wei, L. Zhang, Q. Liu, Y. Luo, T. Li, S. Lu, X. Shi, S. Gao, A. M. Asiri, Z. Wang and X. Sun, Adv. Funct. Mater., 2021, 31, 2009449.

344 Y. Y. Birdja, E. Pérez-Gallent, M. C. Figueiredo, A. J. Göttle, F. Calle-Vallejo and M. T. M. Koper, Nat. Energy, 2019, 4, 732-745.

345 H. Yoshio, K. Katsuhei, M. Akira and S. Shin, Chem. Lett., 1986, 897-898.

346 E. Benson, C. P. Kubiak, A. J. Sathrum and J. M. Smieja, Chem. Soc. Rev., 2009, 38, 89-99.

347 J. Qiao, Y. Liu, F. Hong and J. Zhang, Chem. Soc. Rev., 2014, 43, 631-675.

348 X. Sun, X. Kang, Q. Zhu, J. Ma, G. Yang, Z. Liu and B. Han, Chem. Sci., 2016, 7, 2883-2887.

349 O. S. Bushuyev, P. De Luna, C. T. Dinh, L. Tao, G. Saur, J. van de Lagemaat, S. O. Kelley and E. H. Sargent, Joule, 2018, 2, 825-832.

350 R. J. Lim, M. Xie, M. A. Sk, J.-M. Lee, A. Fisher, X. Wang and K. H. Lim, Catal. Today, 2014, 233, 169-180.
351 Z. Wang, Y. Li, H. Yu, Y. Xu, H. Xue, X. Li, H. Wang and L. Wang, ChemSusChem, 2018, 11, 3480-3485.

352 M.-M. Shi, D. Bao, B.-R. Wulan, Y.-H. Li, Y.-F. Zhang, J.-M. Yan and Q. Jiang, Adv. Mater., 2017, 29, 1606550.

353 S.-J. Li, D. Bao, M.-M. Shi, B.-R. Wulan, J.-M. Yan and Q. Jiang, Adv. Mater., 2017, 29, 1700001.

354 M. Wang, S. Liu, T. Qian, J. Liu, J. Zhou, H. Ji, J. Xiong, J. Zhong and C. Yan, Nat. Commun., 2019, 10, 341.

355 Y. Yao, Q. Feng, S. Zhu, J. Li, Y. Yao, Y. Wang, Q. Wang, M. Gu, H. Wang, H. Li, X.-Z. Yuan and M. Shao, Small Methods, 2019, 3, 1800324.

356 R. Manjunatha, A. Karajić, V. Goldstein and A. Schechter, ACS Appl. Mater. Interfaces, 2019, 11, 7981-7989.

357 P. Wei, H. Xie, X. Zhu, R. Zhao, L. Ji, X. Tong, Y. Luo, G. Cui, Z. Wang and X. Sun, ACS Sustainable Chem. Eng., 2020, 8, 29-33.

358 X. Zhao, X. Lan, D. Yu, H. Fu, Z. Liu and T. Mu, Chem. Commun., 2018, 54, 13010-13013.

359 R. Zhang, L. Ji, W. Kong, H. Wang, R. Zhao, H. Chen, T. Li, B. Li, Y. Luo and X. Sun, Chem. Commun., 2019, 55, 5263-5266.

360 L. Xia, X. Wu, Y. Wang, Z. Niu, Q. Liu, T. Li, X. Shi, A. M. Asiri and X. Sun, Small Methods, 2019, 3, 1800251.

361 R. Francke, B. Schille and M. Roemelt, Chem. Rev., 2018, 118, 4631-4701.

362 Y. Wang, J. Liu, Y. Wang, A. M. Al-Enizi and G. Zheng, Small, 2017, 13, 1701809.

363 R. Schlögl, Angew. Chem., Int. Ed., 2015, 54, 3465-3520.

364 J. Schneider, H. Jia, J. T. Muckerman and E. Fujita, Chem. Soc. Rev., 2012, 41, 2036-2051.

365 E. V. Kondratenko, G. Mul, J. Baltrusaitis, G. O. Larrazábal and J. Pérez-Ramírez, Energy Environ. Sci., 2013, 6, 3112-3135.

366 J. Albo, M. Alvarez-Guerra, P. Castaño and A. Irabien, Green Chem., 2015, 17, 2304-2324.

367 M. F. Baruch, J. E. Pander, J. L. White and A. B. Bocarsly, ACS Catal., 2015, 5, 3148-3156.

368 J. E. Pander, M. F. Baruch and A. B. Bocarsly, ACS Catal., 2016, 6, 7824-7833.

369 A. Dutta, A. Kuzume, M. Rahaman, S. Vesztergom and P. Broekmann, ACS Catal., 2015, 5, 7498-7502.

370 C. Cui, J. Han, X. Zhu, X. Liu, H. Wang, D. Mei and Q. Ge, J. Catal., 2016, 343, 257-265.

371 J. T. Feaster, C. Shi, E. R. Cave, T. Hatsukade, D. N. Abram, K. P. Kuhl, C. Hahn, J. K. Nørskov and T. F. Jaramillo, ACS Catal., 2017, 7, 4822-4827.

372 J. L. White and A. B. Bocarsly, J. Electrochem. Soc., 2016, 163, H410-H416.

373 S. Back, J.-H. Kim, Y.-T. Kim and Y. Jung, Phys. Chem. Chem. Phys., 2016, 18, 9652-9657.

374 J. H. Koh, D. H. Won, T. Eom, N.-K. Kim, K. D. Jung, H. Kim, Y. J. Hwang and B. K. Min, ACS Catal., 2017, 7, 5071-5077.

375 J. S. Yoo, R. Christensen, T. Vegge, J. K. Nørskov and F. Studt, ChemSusChem, 2016, 9, 358-363.

376 N. J. Firet and W. A. Smith, ACS Catal., 2017, 7, 606-612. 
377 S. Back, M. S. Yeom and Y. Jung, ACS Catal., 2015, 5, 5089-5096.

378 T. Cheng, Y. Huang, H. Xiao and W. A. Goddard, J. Phys. Chem. Lett., 2017, 8, 3317-3320.

379 J. Rosen, G. S. Hutchings, Q. Lu, S. Rivera, Y. Zhou, D. G. Vlachos and F. Jiao, ACS Catal., 2015, 5, 4293-4299.

380 H.-E. Lee, K. D. Yang, S. M. Yoon, H.-Y. Ahn, Y. Y. Lee, H. Chang, D. H. Jeong, Y.-S. Lee, M. Y. Kim and K. T. Nam, ACS Nano, 2015, 9, 8384-8393.

381 A. D. Handoko, K. W. Chan and B. S. Yeo, ACS Energy Lett., 2017, 2, 2103-2109.

382 E. Pérez-Gallent, M. C. Figueiredo, F. Calle-Vallejo and M. T. M. Koper, Angew. Chem., Int. Ed., 2017, 56, 3621-3624.

383 J. D. Goodpaster, A. T. Bell and M. Head-Gordon, J. Phys. Chem. Lett., 2016, 7, 1471-1477.

384 D. Ren, B. S.-H. Ang and B. S. Yeo, ACS Catal., 2016, 6, 8239-8247.

385 S. Lee, G. Park and J. Lee, ACS Catal., 2017, 7, 8594-8604. 386 S. Gao, Y. Lin, X. Jiao, Y. Sun, Q. Luo, W. Zhang, D. Li, J. Yang and Y. Xie, Nature, 2016, 529, 68-71.

387 S. Ma, M. Sadakiyo, R. Luo, M. Heima, M. Yamauchi and P. J. A. Kenis, J. Power Sources, 2016, 301, 219-228.

388 F. Calle-Vallejo and M. T. M. Koper, Angew. Chem., Int. Ed., 2013, 52, 7282-7285.

389 Y. Huang, A. D. Handoko, P. Hirunsit and B. S. Yeo, ACS Catal., 2017, 7, 1749-1756.

390 C. Hahn, T. Hatsukade, Y.-G. Kim, A. Vailionis, J. H. Baricuatro, D. C. Higgins, S. A. Nitopi, M. P. Soriaga and T. F. Jaramillo, Proc. Natl. Acad. Sci. U. S. A., 2017, 114, 5918.

391 H.-R. M. Jhong, S. Ma and P. J. A. Kenis, Curr. Opin. Chem. Eng., 2013, 2, 191-199.

392 X. Duan, J. Xu, Z. Wei, J. Ma, S. Guo, S. Wang, H. Liu and S. Dou, Adv. Mater., 2017, 29, 1701784.

393 A. Loiudice, P. Lobaccaro, E. A. Kamali, T. Thao, B. H. Huang, J. W. Ager and R. Buonsanti, Angew. Chem., Int. Ed., 2016, 55, 5789-5792.

394 M. B. Ross, C. T. Dinh, Y. Li, D. Kim, P. De Luna, E. H. Sargent and P. Yang, J. Am. Chem. Soc., 2017, 139, 9359-9363.

395 M. M. Abdelnaby, K. Liu, K. Hassanein and Z. Yin, ChemNanoMat, 2021, 7, 969-981.

396 S. Nitopi, E. Bertheussen, S. B. Scott, X. Liu and I. Chorkendorff, Chem. Rev., 2019, 119, 7610-7672.

397 B. Jiang, X.-G. Zhang, K. Jiang, D.-Y. Wu and W.-B. Cai, J. Am. Chem. Soc., 2018, 140, 2880-2889.

398 H. S. Jeon, I. Sinev, F. Scholten, N. J. Divins, I. Zegkinoglou, L. Pielsticker and B. R. Cuenya, J. Am. Chem. Soc., 2018, 140, 9383-9386.

399 S. Y. Lee, H. Jung, N.-K. Kim, H.-S. Oh, B. K. Min and Y. J. Hwang, J. Am. Chem. Soc., 2018, 140, 8681-8689.

400 M. Ma, B. J. Trześniewski, J. Xie and W. A. Smith, Angew. Chem., Int. Ed., 2016, 55, 9748-9752.

401 Y.-X. Duan, F.-L. Meng, K.-H. Liu, S.-S. Yi, S.-J. Li, J.-M. Yan and Q. Jiang, Adv. Mater., 2018, 30, 1706194.
402 R. M. Arán-Ais, F. Scholten, S. Kunze, R. Rizo and B. Roldan Cuenya, Nat. Energy, 2020, 5, 317-325.

403 J. Xie, X. Zhao, M. Wu, Q. Li, Y. Wang and J. Yao, Angew. Chem., 2018, 130, 9788-9792.

404 T. T. H. Hoang, S. Verma, S. Ma, T. T. Fister, J. Timoshenko, A. I. Frenkel, P. J. A. Kenis and A. A. Gewirth, J. Am. Chem. Soc., 2018, 140, 5791-5797.

405 E. L. Clark, C. Hahn, T. F. Jaramillo and A. T. Bell, J. Am. Chem. Soc., 2017, 139, 15848-15857.

406 J. T. L. Gamler, H. M. Ashberry, S. E. Skrabalak and K. M. Koczkur, Adv. Mater., 2018, 30, 1801563.

407 D. Kim, C. Xie, N. Becknell, Y. Yu, M. Karamad, K. Chan, E. J. Crumlin, J. K. Nørskov and P. Yang, J. Am. Chem. Soc., 2017, 139, 8329-8336.

408 S. Ma, M. Sadakiyo, M. Heima, R. Luo, R. T. Haasch, J. I. Gold, M. Yamauchi and P. J. A. Kenis, J. Am. Chem. Soc., 2017, 139, 47-50.

409 X. Zhang, F. Li, Y. Zhang, A. M. Bond and J. Zhang, J. Mater. Chem. A, 2018, 6, 7851-7858.

410 H. B. Yang, S.-F. Hung, S. Liu, K. Yuan, S. Miao, L. Zhang, X. Huang, H.-Y. Wang, W. Cai, R. Chen, J. Gao, X. Yang, W. Chen, Y. Huang, H. M. Chen, C. M. Li, T. Zhang and B. Liu, Nat. Energy, 2018, 3, 140-147.

411 X. Wang, Z. Chen, X. Zhao, T. Yao, W. Chen, R. You, C. Zhao, G. Wu, J. Wang, W. Huang, J. Yang, X. Hong, S. Wei, Y. Wu and Y. Li, Angew. Chem., 2018, 130, 1962-1966.

412 Y. Pan, R. Lin, Y. Chen, S. Liu, W. Zhu, X. Cao, W. Chen, K. Wu, W.-C. Cheong, Y. Wang, L. Zheng, J. Luo, Y. Lin, Y. Liu, C. Liu, J. Li, Q. Lu, X. Chen, D. Wang, Q. Peng, C. Chen and Y. Li, J. Am. Chem. Soc., 2018, 140, 4218-4221.

413 Y. Cheng, S. Zhao, B. Johannessen, J.-P. Veder, M. Saunders, M. R. Rowles, M. Cheng, C. Liu, M. F. Chisholm, R. De Marco, H.-M. Cheng, S.-Z. Yang and S. P. Jiang, Adv. Mater., 2018, 30, 1706287.

414 N. M. Adli, W. T. Shan, S. Hwang, W. Samarakoon, S. Karakalos, Y. Li, D. A. Cullen, D. Su, Z. X. Feng, G. F. Wang and G. Wu, Angew. Chem., Int. Ed., 2021, 60, 1022-1032.

415 H. Jin, C. Guo, X. Liu, J. Liu, A. Vasileff, Y. Jiao, Y. Zheng and S.-Z. Qiao, Chem. Rev., 2018, 118, 6337-6408.

416 W. Zhang, Q. Qin, L. Dai, R. Qin, X. Zhao, X. Chen, D. Ou, J. Chen, T. T. Chuong, B. Wu and N. Zheng, Angew. Chem., Int. Ed., 2018, 57, 9475-9479.

417 C. Cao, D.-D. Ma, J.-F. Gu, X. Xie, G. Zeng, X. Li, S.-G. Han, Q.-L. Zhu, X.-T. Wu and Q. Xu, Angew. Chem., Int. Ed., 2020, 59, 15014-15020.

418 F. P. García de Arquer, O. S. Bushuyev, P. De Luna, C.-T. Dinh, A. Seifitokaldani, M. I. Saidaminov, C.-S. Tan, L. N. Quan, A. Proppe, M. G. Kibria, S. O. Kelley, D. Sinton and E. H. Sargent, Adv. Mater., 2018, 30, 1802858.

419 W. Bi, C. Wu and Y. Xie, ACS Energy Lett., 2018, 3, 624-633. 420 J. Xu, X. Li, W. Liu, Y. Sun, Z. Ju, T. Yao, C. Wang, H. Ju, J. Zhu, S. Wei and Y. Xie, Angew. Chem., Int. Ed., 2017, 56, 9121-9125.

421 N. Elgrishi, M. B. Chambers, X. Wang and M. Fontecave, Chem. Soc. Rev., 2017, 46, 761-796. 
422 N. Han, Y. Wang, L. Ma, J. Wen, J. Li, H. Zheng, K. Nie, X. Wang, F. Zhao, Y. Li, J. Fan, J. Zhong, T. Wu, D. J. Miller, J. Lu, S.-T. Lee and Y. Li, Chem, 2017, 3, 652-664.

423 W.-H. Wang, Y. Himeda, J. T. Muckerman, G. F. Manbeck and E. Fujita, Chem. Rev., 2015, 115, 12936-12973.

424 W. Zhang, W. Lai and R. Cao, Chem. Rev., 2017, 117, 3717-3797.

425 X. Zhang, Z. Wu, X. Zhang, L. Li, Y. Li, H. Xu, X. Li, X. Yu, Z. Zhang, Y. Liang and H. Wang, Nat. Commun., 2017, 8, 14675.

426 M. Wang, L. Chen, T.-C. Lau and M. Robert, Angew. Chem., Int. Ed., 2018, 57, 7769-7773.

427 O. G. Sánchez, Y. Y. Birdja, M. Bulut, J. Vaes, T. Breugelmans and D. Pant, Curr. Opin. Green Sust., 2019, 16, 47-56.

428 S. Zhang, P. Kang, S. Ubnoske, M. K. Brennaman, N. Song, R. L. House, J. T. Glass and T. J. Meyer, J. Am. Chem. Soc., 2014, 136, 7845-7848.

429 H. Wang, Y. Chen, X. Hou, C. Ma and T. Tan, Green Chem., 2016, 18, 3250-3256.

430 K. Nakata, T. Ozaki, C. Terashima, A. Fujishima and Y. Einaga, Angew. Chem., Int. Ed., 2014, 53, 871-874.

431 K. Natsui, H. Iwakawa, N. Ikemiya, K. Nakata and Y. Einaga, Angew. Chem., Int. Ed., 2018, 57, 2639-2643.

432 X. Xue, H. Yang, T. Yang, P. Yuan, Q. Li, S. Mu, X. Zheng, L. Chi, J. Zhu, Y. Li, J. Zhang and Q. Xu, J. Mater. Chem. A, 2019, 7, 15271-15277.

433 T. Liu, S. Ali, Z. Lian, C. Si, D. S. Su and B. Li, J. Mater. Chem. A, 2018, 6, 19998-20004.

434 H. Yang, Y. Wu, Q. Lin, L. Fan, X. Chai, Q. Zhang, J. Liu, C. He and Z. Lin, Angew. Chem., Int. Ed., 2018, 57, 15476-15480.

435 G.-L. Chai and Z.-X. Guo, Chem. Sci., 2016, 7, 1268-1275.

436 H. Wang, J. Jia, P. Song, Q. Wang, D. Li, S. Min, C. Qian, L. Wang, Y. F. Li, C. Ma, T. Wu, J. Yuan, M. Antonietti and G. A. Ozin, Angew. Chem., Int. Ed., 2017, 56, 7847-7852.

437 J. Wu, M. Liu, P. P. Sharma, R. M. Yadav, L. Ma, Y. Yang, X. Zou, X.-D. Zhou, R. Vajtai, B. I. Yakobson, J. Lou and P. M. Ajayan, Nano Lett., 2016, 16, 466-470.

438 J. Wu, S. Ma, J. Sun, J. I. Gold, C. Tiwary, B. Kim, L. Zhu, N. Chopra, I. N. Odeh, R. Vajtai, A. Z. Yu, R. Luo, J. Lou, G. Ding, P. J. A. Kenis and P. M. Ajayan, Nat. Commun., 2016, 7, 13869.

439 Y. Song, W. Chen, C. Zhao, S. Li, W. Wei and Y. Sun, Angew. Chem., Int. Ed., 2017, 56, 10840-10844.
440 Y. Liu, Y. Zhang, K. Cheng, X. Quan, X. Fan, Y. Su, S. Chen, H. Zhao, Y. Zhang, H. Yu and M. R. Hoffmann, Angew. Chem., Int. Ed., 2017, 56, 15607-15611.

441 X.-M. Hu, H. H. Hval, E. T. Bjerglund, K. J. Dalgaard, M. R. Madsen, M.-M. Pohl, E. Welter, P. Lamagni, K. B. Buhl, M. Bremholm, M. Beller, S. U. Pedersen, T. Skrydstrup and K. Daasbjerg, ACS Catal., 2018, 8, 6255-6264.

442 X. Sun, L. Lu, Q. Zhu, C. Wu, D. Yang, C. Chen and B. Han, Angew. Chem., Int. Ed., 2018, 57, 2427-2431.

443 W. Ju, A. Bagger, G.-P. Hao, A. S. Varela, I. Sinev, V. Bon, B. Roldan Cuenya, S. Kaskel, J. Rossmeisl and P. Strasser, Nat. Commun., 2017, 8, 944.

444 C. Chen, X. Sun, X. Yan, Y. Wu, H. Liu, Q. Zhu, B. B. A. Bediako and B. Han, Angew. Chem., Int. Ed., 2020, 59, 11123-11129.

445 Y. Liu, S. Chen, X. Quan and H. Yu, J. Am. Chem. Soc., 2015, 137, 11631-11636.

446 L. Ye, Y. Ying, D. Sun, Z. Zhang, L. Fei, Z. Wen, J. Qiao and H. Huang, Angew. Chem., Int. Ed., 2020, 59, 3244-3251.

447 Y. Jiao, Y. Zheng, P. Chen, M. Jaroniec and S.-Z. Qiao, J. Am. Chem. Soc., 2017, 139, 18093-18100.

448 J. Wu, R. M. Yadav, M. Liu, P. P. Sharma, C. S. Tiwary, L. Ma, X. Zou, X.-D. Zhou, B. I. Yakobson, J. Lou and P. M. Ajayan, ACS Nano, 2015, 9, 5364-5371.

449 B. Kumar, M. Asadi, D. Pisasale, S. Sinha-Ray, B. A. Rosen, R. Haasch, J. Abiade, A. L. Yarin and A. Salehi-Khojin, Nat. Commun., 2013, 4, 2819.

450 H. Yin, K. Xing, Y. Zhang, D. M. A. S. Dissanayake, Z. Lu, H. Zhao, Z. Zeng, J.-H. Yun, D.-C. Qi and Z. Yin, Chem. Soc. Rev., 2021, 50, 6423-6482.

451 N. Uddin, H. Zhang, Y. Du, G. Jia, S. Wang and Z. Yin, Adv. Mater., 2020, 32, 1905739.

452 Y. Yu, Y. Shi and B. Zhang, Acc. Chem. Res., 2018, 51, 1711-1721.

453 F. Lai, Z. Sun, S. E. Saji, Y. He, X. Yu, H. Zhao, H. Guo and Z. Yin, Small, 2021, 17, 2100024.

454 O. A. Moses, L. Gao, H. Zhao, Z. Wang, M. Lawan Adam, Z. Sun, K. Liu, J. Wang, Y. Lu, Z. Yin and X. Yu, Mater. Today, 2021, 50, 116-148.

455 M. Zhong, K. Tran, Y. Min, C. Wang, Z. Wang, C.-T. Dinh, P. De Luna, Z. Yu, A. S. Rasouli, P. Brodersen, S. Sun, O. Voznyy, C.-S. Tan, M. Askerka, F. Che, M. Liu, A. Seifitokaldani, Y. Pang, S.-C. Lo, A. Ip, Z. Ulissi and E. H. Sargent, Nature, 2020, 581, 178-183. 